\title{
Experts and idiots
}

Citation for published version (APA):

Moes, F. B. (2019). Experts and idiots: an ethnography of epistemic practices in Dutch social health insurance. [Doctoral Thesis, Maastricht University]. Maastricht University. https://doi.org/10.26481/dis.20190308fm

Document status and date:

Published: 01/01/2019

DOI:

10.26481/dis.20190308fm

Document Version:

Publisher's PDF, also known as Version of record

\section{Please check the document version of this publication:}

- A submitted manuscript is the version of the article upon submission and before peer-review. There can be important differences between the submitted version and the official published version of record.

People interested in the research are advised to contact the author for the final version of the publication, or visit the DOI to the publisher's website.

- The final author version and the galley proof are versions of the publication after peer review.

- The final published version features the final layout of the paper including the volume, issue and page numbers.

Link to publication

\footnotetext{
General rights rights.

- You may freely distribute the URL identifying the publication in the public portal. please follow below link for the End User Agreement:

www.umlib.nl/taverne-license

Take down policy

If you believe that this document breaches copyright please contact us at:

repository@maastrichtuniversity.nl

providing details and we will investigate your claim.
}

Copyright and moral rights for the publications made accessible in the public portal are retained by the authors and/or other copyright owners and it is a condition of accessing publications that users recognise and abide by the legal requirements associated with these

- Users may download and print one copy of any publication from the public portal for the purpose of private study or research.

- You may not further distribute the material or use it for any profit-making activity or commercial gain

If the publication is distributed under the terms of Article $25 \mathrm{fa}$ of the Dutch Copyright Act, indicated by the "Taverne" license above, 




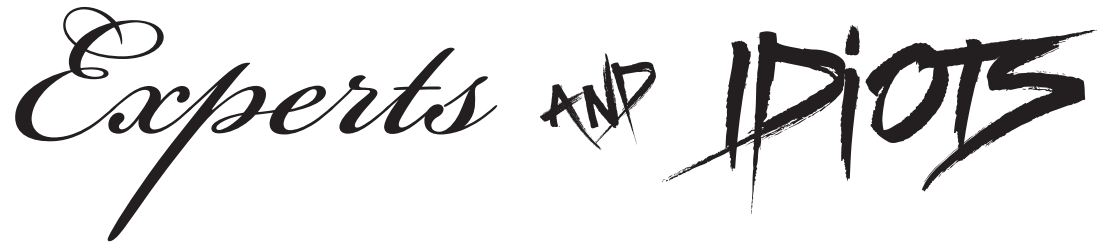

An Ethnography of Epistemic Practices in Dutch Social Health Insurance

Floortje Moes 


\section{COLOPHON}

Cover: Christiaan Maats (www.christiaanmaats.com)

Layout: Legatron Electronic Publishing

Printing: Ipskamp Printing, Enschede (www.ipskampprinting.nl)

ISBN: 978-94-028-1397-5

(C) Floortje Moes 2019. All Rights Reserved. 


\section{Experts and Idiots}

An Ethnography of Epistemic Practices in

Dutch Social Health Insurance

Dissertation

To obtain the degree of Doctor at Maastricht University,

on the authority of the Rector Magnificus, Prof. dr. Rianne M. Letschert in accordance with the decision of the Board of Deans, to be defended in public on Friday March $8^{\text {th }} 2019$ at 16:00 hours by

Floortje Bernadette Moes 


\section{Supervisors}

Prof. Dr. K. Horstman, Maastricht University

Prof. Dr. D. M. J. Delnoij, Erasmus University Rotterdam I Zorginstituut Nederland

Prof. Dr. E. S. Houwaart, Maastricht University

\section{Assessment Committee}

Prof. Dr. D. Ruwaard, Maastricht University (Chair)

Prof. Dr. R. A. Bal, Erasmus University Rotterdam

Prof. Dr. J. W. L. Cals, Maastricht University

Prof. Dr. F. G. Huisman, Maastricht University I University Medical Center Utrecht

Prof. Dr. J. A. M. Kremer, Radboud University Nijmegen

The research presented in this book was conducted at CAPHRI Care and Public Health Research Institute, Department of Health, Ethics and Society of Maastricht University. CAPHRI participates in the Netherlands School of Public Health and Care Research, CaRe.

The research project was conducted as part of an academic collaboration between the Dutch National Health Care Institute (Zorginstituut Nederland) and the Department of Health, Ethics and Society of Maastricht University. 


\section{Contents}

$\begin{array}{llr}\text { Introduction } & \text { Epistemic Practices in Policymaking } & 7 \\ \text { Chapter } \mathbf{1} & \text { Contested Evidence } & 31 \\ \text { Chapter } \mathbf{2} & \text { Strangers in the ER } & 55 \\ \text { Chapter 3 } & \text { Collective Constructions of 'Waste' } & 73 \\ \text { Chapter } \mathbf{4} & \text { 'Epistemic Injustice' in Knowledge-intensive Policymaking } & 99 \\ \text { Conclusions } & \text { Experts and Idiots } & 121 \\ & & \\ \text { Appendices } & \text { Valorization of Research Insights } & 147 \\ & \text { Summary } & 161 \\ & \text { Samenvatting } & 165 \\ & \text { List of Publications } & 169 \\ & \text { Acknowledgements } & 171 \\ & \text { Author Details } & 175\end{array}$





\section{Introduction}

\section{Epistemic Practices in Policymaking}




\section{SCIENCE IN DEMOCRACY}

Immigration policy, national vaccination programs, regulations for privacy, class size in basic education, or the quality and safety of our food; no policy domain is without a correlative field of scientific knowledge that is called upon to substantiate our public choices. Scientific knowledge has come to play a central role in policy decisions. Western democratic culture is largely characterized by this intimate relationship between science and the state. A solid foundation of public policy in the form of scientific evidence or expertise has become an 'expectation against which political actors can be judged' (Parkhurst, 2017, p. 4).

The Dutch Minister for Infrastructure and Environment, for example, backed up plans for the expansion of Lelystad Airport with an Environmental Impact Report made by an independent expert committee. When a citizen action group discovered that there were miscalculations in the committee's report, the minister was forced to redo the calculations on the sound pollution that would result from an increase in flights at Lelystad Airport (de Jonge \& Nijland, 2018; de Zeeuw, 2017). Similarly, the British government was criticized for pursuing immigration restrictions based on public perceptions that immigrants were abusing state benefits and tax revenues, while evidence showed that foreign-born workers were actually less likely to claim benefits than UK nationals (Partos, 2014).

In Western democratic societies it has become a sign of good governance that policymakers substantiate their work with up-to-date expert knowledge; those who fail to do so can expect public criticism and oppositional wrath. Referrals to scientific knowledge are presumed to pertain to ideals of impartiality, objectivity and justice in policymaking. They are supposed to safeguard policymakers against the suspicion of arbitrariness or bias in making public decisions (Porter, 1994, 1995). It is this interplay between expert knowledge and the legitimation of public policy that is the topic of this book. In particular, it addresses the interplay between knowledge and the legitimation of thorny policy decisions in social health insurance. But before moving on to the particular research setting of this book, it is important to first explore in more depth the complexity of the relationship between knowledge and policy.

\section{POLITICIZATION OF SCIENCE}

There is something oddly paradoxical about the relationship between science and policy. While scientific knowledge is ever more solicited in governance, at the same time, the authority of scientific advice is being challenged more often in the public domain (Bijker, Bal, \& Hendriks, 2009; Horstman, 2014). Evidence and expertise that are used to justify policy decisions are often disputed by contra-experts, citizens or journalists. For example, the consensus amongst scientists is that human activity is causing global warming (Hoffman, 2011; Oreskes, 2004). However, since the issue of climate change has become of importance to policymaking, climate change 'denialists' 
and contrarian scientists (allegedly influenced by corporatist interests) started raising doubt about the evidence supporting anthropogenic global warming (Oreskes \& Conway, 2012). They claim that scientific publications about human-caused climate change are the result of political bias of those in editorial positions on academic journals (Hoffman, 2011). Both sides of the debate are now accusing one another of biased selection of evidence (Parkhurst, 2017, p. 47). Other examples are not hard to come by: from national vaccination programs (Boyce, 2007) to guidelines on genetically modified organisms (GMOs) in food (Klintman, 2002), policies and regulations are frequently accompanied by controversies about the science supporting them. According to some scholars, this is not surprising as processes of scientization of public policy actually go together with processes of politicization of scientific knowledge (Hoppe, 1999, 2010; Jasanoff, 1990; Lövbrand, 2011; Weingart, 1999).

While the use of scientific evidence has become an expectation against which policymakers' work is assessed, at the same time, evidence has become controversial:

'there is no such entity as "the body of evidence." There are simply (more or less) competing (re)constructions of evidence able to support almost any position. Much of what is called evidence is, in fact, a contested domain, constituted in the debates and controversies of opposing viewpoints in search of ever more compelling arguments.' (Wood, Ferlie, \& Fitzgerald, 1998, p. 1735)

The idea that policy should be based on evidence appears self-evident. The above quote, however, reminds us of two things. First, evidence alone can never tell policymakers what it is that should be done. Evidence is 'multiple' and - in policymaking - it has to be appraised and interpreted through a mesh of political, cultural and social values that surround major policy issues. Evidence-based policymaking is modelled on the rationalist idea that a policy problem can be clearly defined and that research evidence can be used to solve that problem. However, no amount of evidence can ever tell a policymaker whether public money is better spent on improving local schooling, investing in disadvantaged neighborhoods or putting more police on the streets. What benefits the nation the most? Ultimately, these are questions of politics. The idea that the relationship between research evidence and policymaking is linear and rational has long been criticized (Lindblom, 1959; Russell \& Greenhalgh, 2009; Sanderson, 2002; Stone, 2002). Expert knowledge and evidence are often used to justify policy that is - inevitably and justifiably - just as much based on ideological, political, cultural and moral convictions.

Second, the quote reminds us of something more fundamental: 'evidence' is, essentially, a contested concept. Evidence and expertise are often invoked to provide a neutral, rationalistic and technical underpinning of public policy, but although evidence and expertise may seem neutral, in fact, they are not. What constitutes 'evidence' and what counts as 'objective', 'scientific' or 'expert' knowledge is a philosophical question, rooted in the Philosophy of Science. Most $20^{\text {th }}$ century philosophers of science took a normative approach to the question: providing answers as to what 
can and what cannot be counted as scientific knowledge (e.g. Hahn, Neurath, \& Carnap, 1929; Popper, 1935 [1959]; Quine, 1951). Scholars in the field of Science and Technology Studies (STS) developed a more empirical approach in the 70s and 80s. Like anthropologists, these philosophers started studying how science was 'done' in daily scientific practices, looking at scientists as if they were members of a foreign tribe (Collins, 1985; Knorr Cetina, 1981; Latour \& Woolgar, 1979; Lynch, 1985; Traweek, 1988).

For example, in their famous work 'Laboratory Life. The Construction of Scientific Facts', Latour and Woolgar studied the work of scientists investigating the peptide TRF at Roger Guillemin's laboratory at the Salk Institute (Latour \& Woolgar, 1979). They describe scientists' routine practices involving technical instruments and lab rats, scientists' preoccupation with the publication of papers, and their strategic work to gain academic prestige and research funding. It showed how scientific 'facts' are constructed, and that - in that process - scientists are not merely guided by goals of truth finding, but also by personal, social and political interests.

'Laboratory Life' was groundbreaking, because it showed that science is a social domain like any other; it has culture and politics. The book shattered the standard view that science is a neutral place where 'facts' are uncovered by disengaged scientists who are merely acting as a mouthpiece for 'Truth' and 'Nature'. It became apparent that, to a certain extent, facts are man-made, value-laden and the result of hard (and often strategic) work (Latour \& Woolgar, 1979). The anthropological study of science showed that any field of scientific inquiry has its controversies and its internal 'wars'. A myth about science that was systematically dispelled by these science-anthropologists is that facts are simply 'given' by nature and that 'experts can be expected to agree' (Latour, 1987; Weinel, 2008, p. 4).

\section{PROFESSIONAL NORMS OF ‘OBJECTIVITY’ AND PUBLIC ACCOUNTABILITY}

Disputes over what counts as 'objective', 'scientific' or 'expert' knowledge are not exclusive to laboratories, universities and research centers. Conventions and norms of 'objectivity' are created in all forms of professional life (Porter, 1995). In topics ranging from life insurance (Horstman, 2001), office environment toxicity (Murphy, 2006) to atlas images (Daston \& Galison, 1992), empirical work in STS has shown the historical tussles and debates in answering the question of what type of knowledge counts as 'objective' in particular areas of professional life. According to Knorr Cetina, every place that produces knowledge claims is host to 'practices, arrangements and mechanisms' which - in that area of professional expertise - make up 'how we know what we know' (Knorr Cetina, 2007, p. 363). She coined the term 'epistemic culture' to refer to these historically, politically and socially grounded practices of creating and warranting knowledge, and modern societies are permeated with such knowledge settings (Knorr Cetina, 1999, 2007). 
Working methods, techniques or tools of knowing, express an epistemic ideal of 'objectivity' in a particular profession. Such epistemic practices are related to how a profession makes itself publicly accountable for what it is licensed to do. Porter's seminal book 'Trust in Numbers. The Pursuit of Objectivity in Science and Public Life' is one of the most detailed accounts that we have of how epistemic practices are connected to public accountability. Drawing on case studies ranging from accountancy and insurance to civil engineering, Porter shows how throughout Western democracies we have come to rely heavily on quantified knowledge (Porter, 1995).

It was in times of economic and social transformation in the $19^{\text {th }}$ century, that traditional belief in the trustworthiness of elites started to decline, democratic types of governance emerged and bureaucracies developed. In the transition towards an industrial society, traditional face-to-face transactions were replaced by long-distance, and increasingly anonymous, chains of transactions. This meant that people became dependent on persons they did not personally know, in matters ranging from economic transactions to political representation. Quantified knowledge and rulebased procedures developed in this era. In the emergent political culture that idealized the rule of law, it was bad policy to rely merely on the personal judgement of bureaucratic officials:

'Arbitrariness and bias are the most usual grounds upon which such officials are criticized. A decision made by the numbers (or by explicit rules of some other sort) has at least the appearance of being fair and impersonal. Scientific objectivity thus provides an answer to a moral demand for impartiality and fairness. Quantification is a way of making decisions without seeming to decide. Objectivity lends authority to officials who have very little of their own' (Porter, 1995, p. 8)

Numbers are 'more open and less personal' and allow scrutiny and control (Porter, 1995, p. 7). As such, quantitative and procedural forms of accountability have become increasingly important in societies where people no longer rely on intimate knowledge and personal trust, where elites are weak, where private negotiation is suspect and where trust is in short supply (Porter, 1995).

'Quantification is well suited for communication that goes beyond the boundaries of locality and community. [...] In science, as in political and administrative affairs, objectivity names a set of strategies for dealing with distance and distrust' (Porter, 1995, p. ix)

Porter's work on the rise of quantitative knowledge to achieve 'objectivity' in science and public life shows how public accountability and epistemic ideals were mutually constitutive in the historical setting of the $19^{\text {th }}$ century. Porter demonstrates how statistics, numerical facts and figures have become a means to achieve objectivity and public trust in modern scientific, professional and public life. 'Trust in Numbers' invites us to take a closer look at knowledge practices and to understand the forms of trust and accountability that are constituted in them. 
This book investigates contemporary epistemic practices - the preferred scientific methods, dominant notions of evidence, and epistemic ideals of 'objectivity' - of policymakers and experts working on the science-policy nexus. It investigates how public accountability for policy choices is constituted in these practices. Which types of knowledge, expertise or 'evidence' are used to legitimate difficult policy choices? In particular, this study looks at Dutch policymakers working in the field of social health insurance. Amid increasing health care needs and growing budget pressure, the work of these policymakers is an exceptionally daunting task that is morally, socially and politically charged. How do these policymakers employ different forms of knowledge and different types of evidence in order to justify thorny health policy decisions? But before tuning in with the situation in Dutch health care, let us first consider this: why are the epistemic practices of policymakers in need of reflection at all?

\section{WHY STUDY EPISTEMIC PRACTICES IN POLICYMAKING?}

According to Miranda Fricker, there is such a thing as 'epistemic injustice': we can do someone, or a group of people, an injustice by not recognizing them 'in their capacity as knower' (Fricker, 2007, p. 1). Someone can, for example, be wronged in their capacity as 'a giver of knowledge' by ignoring them as an epistemic agent that can provide us with trustworthy and reliable information (Fricker, 2007, p. 7). Fricker calls this 'testimonial injustice'. In 'To Kill a Mockingbird', for example, jurors fail to believe the testimony of Tom Robinson, a black man in Alabama (USA) in 1935, because they are prejudiced against black people (Fricker, 2007, p. 23). When someone lacks credibility, "their assertions are not accepted by those to whom they are directed, and they are treated as lacking what is required to be a reliable informant' (Hookway, 2010, p. 152).

Some have reported examples of testimonial injustices to patients in health care practices (Carel \& Kidd, 2014; Kidd \& Carel, 2017). For example, Carel and Kidd recall a study published in 1984 (Beckman \& Frankel, 1984) that found that the average amount of time between a patient beginning to speak and the doctor's first interruption was eighteen seconds (Kidd \& Carel, 2017). Only $23 \%$ of patients could complete his or her opening statement of concerns, which, according to the authors, resulted in the loss of relevant medical information (Beckman \& Frankel, 1984). As another example, Carel and Kidd describe the following iconic scene that they observed in a hospital setting:

“"That really, really hurts", says the woman who has just given birth. The doctor is sitting by her splayed feet, which rest on stirrups either side of him. He is stitching her vagina, his face inches away from her body. A crowd of doctors and nurses surround the baby lying a few feet away. He is being bundled, rubbed, and his airways cleared by eight health professionals. But none of them seem to hear, or respond to, the woman's complaint. She repeats: "That hurts. Are you using anaesthetic?". "No", the doctor replies 
calmly, "there is no need to. I am nearly finished". The woman is too exhausted to persist and she says nothing more. It is hard to imagine another situation in which we would not offer pain relief to someone having a needle pushed through their genitals. But in this case the woman's testimony is not acted upon. Her pain is either not fully registered or not considered worthy of response.' (Carel \& Kidd, 2014, p. 529)

Both cases show patients that are not considered relevant informants with regard to their medical situation. According to Fricker, someone's status as epistemic agent affects his/her 'social power', which she defines as the 'capacity we have as social agents to influence how things go in the social world' (Fricker, 2007, p. 9). Fricker considered this a form of injustice, because this person is unable to perform certain social and political tasks that demand credibility.

The idea of 'testimonial injustice' raises questions in the context of policymaking: whose knowledge is recognized as a credible contribution to the policymaking process? In response to claims of a disengaged and disillusioned citizenry, policymakers have been experimenting with deliberative spaces to engage citizens more directly in setting the direction for policy decisions (Dieleman, 2015). Concerns are raised, however, about the 'epistemological status' that is actually accredited to public knowledge (Irwin, 2006, p. 315), and whether a public voice is (unintentionally) being 'made' or 'wrongly appropriated' in such 'formalized mechanisms of voice' (Felt \& Fochler, 2010; Irwin, 2006; Michael, 2012, p. 541). In the context of healthcare policy, it has been argued that patients do not always have the epistemic resources or capacity to 'effectively make use of' the possibilities offered to participate in formal decision-making procedures, and that procedures are not always designed to enable 'more than symbolic participation' (Peeters, Delnoij, \& Friele, 2014 , p. 60). The idea of 'testimonial injustice' in policymaking raises questions with regard to the social inclusiveness of our policymaking practices. An investigation into the epistemic practices of policymakers, allows us to reflect on the adequacy of decision-making processes in terms of social representativeness.

Fricker recognizes another type of epistemic injustice: 'hermeneutical injustice' (Fricker, 2007). This epistemic wrongdoing occurs when 'a gap in collective interpretive resources puts someone at an unfair disadvantage when it comes to making sense of their social experiences' (Fricker, 2007, p. 1). While in 'testimonial' injustice someone is wronged in their capacity as 'a giver of knowledge' (Fricker, 2007, p. 7) and suffers a 'credibility deficit' (Coady, 2010, p. 110), in 'hermeneutical' injustice someone is wronged in their capacity as 'a subject of social understanding' (Fricker, 2007, p. 7) and suffers an 'intelligibility deficit' (Coady, 2010, p. 110). As an example Fricker recounts the situation of Carmita Wood, who suffers sexual harassment prior to the time when we had this critical concept: 'so she cannot properly comprehend her own experience, let alone render it communicatively intelligible to others' (Fricker, 2007, p. 1). This is considered a form of injustice, because someone who cannot make him/herself understood is unable to perform social and political tasks that demand their intelligible contribution. Someone who suffers a lack of conceptual resources to make him/ 
herself understood, is unable to effectively participate in 'practices through which social meanings are generated' (Fricker, 2007, p. 6).

Murphy's research of the coming into being in the 80 s of the 'Sick Building Syndrome' can function as an intriguing example of hermeneutical injustice (although Murphy herself does not necessarily call it by that name) (Murphy, 2006). Murphy shows how a workplace-related constellation of health problems - nausea, headaches, dizziness, sore eyes, rashes, respiratory congestion, and immune system disorders - was long considered a 'hysterical' experience of female office workers. Recognition of this health issue was dependent on how specific measuring techniques made hazardous toxin-levels in office buildings visible. State agencies such as Occupational Health and Safety demanded measurable toxin-levels. However, their experience and tools for measuring toxicity in traditional industrial environments did not pick up the low levels of hazardous toxins present in the modern office environment; what cannot be measured does not exist. Office workers (mostly female) were, as a result, not believed to be experiencing any real health issues. Ultimately, laypersons started conducting extensive social surveys amongst office workers in order to map health problems and to correlate health issues to indoor office environments.

Murphy's analysis shows how certain styles (scientific, corporate or lay approaches) of measuring chemical exposures in office buildings made this health issue visible or invisible, perceptible or imperceptible ${ }^{1}$. As soon as definite pollutants were identified - chemicals in office carpets, paints, photocopy chemicals, emissions from personal computers printers, or bad airconditioning - the workplace-related health problems became 'real', recognized and actionable. Just like Fricker's example of Carmita Wood, these female office workers, for a long time, could not properly comprehend their own experience, or render it communicatively intelligible to those that could potentially improve their situation, for example, office management, health organizations or state officials. This is what it means when 'a gap in collective interpretive resources' (or a lack of adequate epistemic practices) puts someone or some group at an unfair disadvantage in making sense of their social experiences (Fricker, 2007, p. 1).

The idea of 'epistemic injustice' - both testimonial and hermeneutical - seems particularly relevant in the context of policymaking, because someone (or some group) who is not recognized in his/her capacity as an epistemic agent, or who does not have the interpretative resources to make him/herself intelligible, lacks the social power to influence public policy. If people lack the power to influence policy processes, they are potentially deprived of a fair share of our collective (financial, medical, social, political) resources and are excluded from practices of deliberating and constructing our collective futures. The idea of 'epistemic injustice' raises questions: whose knowledge is recognized as credible and intelligible contribution to the policymaking process? An investigation into the currently preferred scientific methods, dominant notions of evidence and

\footnotetext{
${ }^{1}$ Corporate stakeholders such as the tobacco industry profited from the idea that it was the office buildings and their synthetic interiors that were the cause of indoor toxicity. By making the interiors visible as the culprit of office building toxicity, the role of the tobacco industry itself as the producer of unhealthy products causing indoor pollution was, at the same time, obscured and made less visible.
} 
policymakers' epistemic ideals of 'objectivity', allows us to reflect on their adequacy in terms of social representativeness. Do different groups of knowers have the interpretative resources to make themselves understood and intelligible in the decision-making process? Analyzing policy practices from the perspective of epistemic injustice facilitates a search for ways to improve the social inclusiveness and representativeness of policymaking practices.

Next, I will introduce the Dutch health care system and the work of the National Health Care Institute that form the context of this book's case studies. In the methods section I will elaborate on my ethnographic work at the National Health Care Institute and give a rationale for case selection. At the end of this introduction you will find an outline of this book, briefly describing the content of the different chapters and what can be expected from the concluding chapter. I will conclude this introduction by saying something about the title of this book.

\section{GOVERNANCE ON ACCESS, EFFICIENCY AND QUALITY IN DUTCH SOCIAL HEALTH INSURANCE}

The Netherlands has, traditionally, a strong welfare state in which the government has major constitutional responsibilities for the accessibility, efficiency and quality of health care. While the Dutch state has these legal tasks with regard to the nation's health care system, at the same time, an important feature of the policymaking system in the Netherlands is 'an absence of a power center for implementing major policies' (Berg \& Van der Grinten, 2003, p. 118). This has much to do with the corporatist characteristics of how the Dutch health care system is financed and delivered.

Dutch health care is primarily financed through a social health insurance system². The Health Insurance Act obliges everybody who resides - or pays payroll tax - in the Netherlands to take out basic health insurance from a private insurance company. Benefits covered from this basic insurance policy are broadly indicated by law. Citizens can choose between competing health insurance companies during a yearly open enrollment period. Income-related subsidies make basic healthcare insurance affordable for all citizens. Insurers are obliged to accept enrollees regardless

\footnotetext{
${ }^{2}$ The Dutch health insurance scheme was installed during the Second World War by German occupational forces in 1941. Since then, the system has undergone several reforms (Helderman, De Kruijf, Verheij, \& Van Thiel, 2014; Helderman, Schut, van der Grinten, \& van de Ven, 2005; Van de Ven \& Schut, 2008). Initially, health insurance was organized as an employee's insurance: the Sickness Funds. It distinguished between compulsory social health care insurance (for low-income and middle-income groups), and private health insurances (for those above a certain income threshold) (Helderman et al., 2014). Faced with growing budget problems in the 80s, the lack of incentives for efficiency and innovation within the Sickness Funds system raised concerns, leading to growing support for 'incentive-based reforms and a reconsideration of the role of competition' (Van de Ven \& Schut, 2008). Since the 90s successive governments worked to install all conditions necessary for managed competition in the health care system. In 2006, these efforts culminated in a major market-oriented reform within the context of a social health insurance system: the Health Insurance Act (Zorgverzekeringswet, Zvw). The introduction of the Health Insurance Act entailed a transition from government regulation of social health insurance taken out of wages (Sickness Fund system) towards a system of regulated competition between private insurance companies.
} 
of their age or health condition, while a risk adjustment scheme compensates them for clients with predictably high medical expenses (Van de Ven \& Schut, 2009). Private insurance companies are expected to negotiate the prices, services, and quality of care with health care providers on behalf of their insured clients (Van de Ven \& Schut, 2008, p. 779). Insurers and hospitals are free to negotiate prices and selectively contract a range of hospital care products. Insurers can steer their customers away from health care providers that do not reduce their prices or improve quality. This type of 'selective contracting' is thought to stimulate both quality and efficiency in health care (Bijlsma, Boone, \& Zwart, 2009).

Health care is delivered by largely self-employed medical professionals and private (mostly non-profit) health service organizations. General practitioners and medical specialists are commonly private entrepreneurs organized in mutual group practices or partnerships. They enjoy strong professional autonomy in the Netherlands. Primary, specialist and long-term care providers as well as patient groups are well-organized in (national) associations that have significant authority in political and public deliberation. Apart from the organization of the supplyside of healthcare, patients have organized themselves professionally at different levels in the Netherlands: disease-specific patient organizations for individual patients (e.g. breast cancer or Alzheimer's associations), larger umbrella organizations (e.g. Dutch Cancer Society) and regional and national umbrella organizations (such as the Netherlands Patient/Consumer Federation) (Van De Bovenkamp, Trappenburg, \& Grit, 2010). The professional patient organizations, as so-called third-parties alongside care providers and insurers, have increasingly become part of the Dutch decision-making structure as representatives of patients' interests.

Due to the corporatist characteristics of Dutch health care, policymaking in the Netherlands relies strongly on cooperation on a national level between the state and professionals organizations of health care providers, insurers and patients (Berg \& Van der Grinten, 2003; Bolt, 2015). The Dutch consensual way of policy-making (the 'polder model') entails that state actors and field parties reconcile private interests with public interest, and share political authority and responsibility for policy decisions (Helderman et al., 2005; Hemerijck, 1993, p. 192). Whenever difficult decisions in health care have to be made, for example priority-setting or budget planning, their social acceptance and implementation in practice depends on the cooperation of a myriad of stakeholders working at a macro level (e.g. governmental organizations, national professional associations), meso level (e.g. insurers and health care organizations) and micro level (e.g. individual care providers). Especially in these times of soaring health care expenses and limited budgetary elasticity, policymakers who are charged with organizing accessibility, efficiency and quality of Dutch health care, face a difficult task. With diverging interests at stake, it is important that these decisions are well-substantiated and explicitly justified. 


\section{THE ROLE OF EBM IN DUTCH HEALTH GOVERNANCE}

Evidence Based Medicine (EBM) gained a particularly firm foothold as an epistemic ideal for decision-making within the corporatist health care system of the Netherlands (Bolt, 2015). EBM entails 'the conscientious, explicit, and judicious use of current best evidence' in medical decisionmaking (Sackett, Rosenberg, Muir Gray, Haynes, \& Richardson, 1996). EBM was announced in 1992 as a 'new paradigm' for medicine (Evidence-Based Medicine Working Group, 1992, p. 2420), emphasizing the use of experimental evidence in medical decision-making. EBM makes use of a hierarchical classification of medical knowledges according to rigorousness and quality of research methods, with randomized controlled trials (RCTs) and systematic reviews forming the apex of the hierarchy (Guyatt et al., 2008; Guyatt et al., 1995).

Originally designed for clinical practice, EBM has become an epistemic ideal for making policy decisions too: policy should be based on (experimental) evidence of 'what works'. EBM, providing the kind of 'objective', technical and neutral language used by the medical profession itself, helped to transcend different interests and substantiate, explicate and justify difficult prioritysetting decisions, especially in the Netherlands (Bolt, 2015).

The logic of EBM has also come to play an important role in quality management in the context of the Dutch health care market. Transparency and quality information are required as both consumers and insurers need such comparative information about the performance of healthcare providers in order to negotiate price and quality and to facilitate (regulated) competition in health care (Delnoij, Rademakers, \& Groenewegen, 2010; Enthoven \& Van de Ven, 2007). Quality indicators provide a means for health care providers, insurers and policymakers to measure, compare and improve the quality of care (Campbell, Roland, \& Buetow, 2000; Groene, Skau, \& Frølich, 2008; Hilarion et al., 2009). Although quality indicators are not directly linked to the clinical literacy movement that EBM originally set out to be (Djulbegovic \& Guyatt, 2017; Sackett et al., 1996), some have called indicators a 'branch' of EBM (Eddy, 2005) as they follow the same logic: clinical science can determine 'what works' and parameters based on these scientific findings can form an 'objective' standard for provider behavior (Tanenbaum, 1995, 2012).

The epistemic practice of EBM has become pervasive in decision-making at macro-, mesoand micro-levels of the Dutch health care arena since the 1990s (Bolt, 2015). EBM has come to be seen as an 'objective' epistemic practice that has, over the past decades, been instrumental in aligning the government and the medical profession whenever decisions about efficiency, accessibility, quality in health care had to be made (Beyens \& Bolt, 2017; Bolt, 2015). 


\section{THE NATIONAL HEALTH CARE INSTITUTE}

This book focuses on the epistemic practices (and the role of EBM therein) of one of the major players in organizing accessibility, efficiency and quality of Dutch health care: The National Health Care Institute (Zorginstituut Nederland). The Institute is an independent governing agency that falls under the responsibility of the Ministry of Health and is tasked with management of the basic benefits package. Building on almost 70 years of experience and expertise with regard to the statutory health insurance system ${ }^{3}$, the Institute is an authoritative voice in the Dutch health care arena (Helderman et al., 2014). The Minister formally requests advice from the Institute on the medical-technical content of care that can be reimbursed from basic health insurance. The Institute also subjects major new drugs to cost-effectiveness analysis before coverage is considered (Staal, Heymans, Ligtenberg, Derksen, \& Couwenbergh, 2015). Such evaluations avoid the automatic inclusion in the benefits package of new drugs for intramural patients (Helderman et al., 2014, pp. 23-25). The Institute provides authoritative standpoints on existing medical treatments and nonpharmaceutical interventions when clarity is lacking among healthcare professionals about the reimbursement of these treatments.

Since 2014, in addition to its tasks relating to the statutory basic benefits package, the Institute has also lawfully been tasked with providing understandable, comparable and trustworthy information about the quality of care. In the years that followed the introduction of the Health Insurance Act (2006), the health care sector developed all kinds of quality information. The multiplicity of indicators, however, actually blocked oversight and hindered informed consumer choice. In response to this problem, the Minister of Health proposed to 'bundle the organizations into a single national institute that takes the helm and steers decisively towards both the quality and the effectiveness of health care' (Letter to Parliament, May 28 2010). The National Health Care Institute was lawfully tasked to organize quality information, and has what is called 'overriding authority': it can authoritatively lay down quality standards or indicators when private parties fail to deliver them or to reach consensus. Furthermore, the Institute promotes the development of quality measures (e.g. quality standards, clinical guidelines, performance indicators, etc.) and stimulates the implementation of quality metrics.

These are no easy tasks. With the setting of a quality standard, or with the inclusion or exclusion of a specific medical treatment from the basic benefits package, the interests of insurers, health care providers, patients and the public at large are at stake. The Institute has increasingly legitimatized package decisions through the 'scientization of the decision-making process' (Niezen-van der Zwet, 2012, p. 8). Not surprisingly, in view of the wider policymaking culture in the Netherlands, the principles of EBM have come to play an important role in substantiating and justifying package decisions, casting difficult decisions in technical terms of clinical 'effectiveness'

\footnotetext{
${ }^{3}$ The Institute is the successor of the Health Care Insurance Board (CVZ, College voor Zorgverzekering, 1999-2014) and the Health Insurance Council (Zfr, Ziekenfondsraad, 1949-1999) that conducted similar tasks.
} 
(Staal et al., 2015; Staal, Ligtenberg, \& van Drooge-van Loon, 2007). Due to the public nature of the Institute, its knowledge work centers on the technical justification of decisions.

The Institute, nonetheless, experiences difficulties explaining the foundations of these decisions to a wider public. The public outrage in 2012 ensuing the Institute's 'evidence-based' advice to stop the reimbursement of orphan drugs for the rare diseases of Pompe and Fabry is the most prominent example of this (Boon, Martins, \& Koopmanschap, 2015; Schinkelshoek \& Martini, 2012; Van den Brink, 2014). The national daily news broadcast reported Pompe and Fabry patients demonstrating at the National Health Care Institute holding signs saying: "I am too expensive and get a death sentence" (NOS, 2012). Moreover, in 2013, the Patients Association for Interstitial Cystitis sued the Institute for its decision to stop the reimbursement of a treatment that these patients claimed to benefit from. The court case evolved mostly around the question of whether the EBM-method was adequate for making reimbursement decisions on a national level. Also in the context of quality management, evidence can easily be contested. For example, in 2013, the 'evidence-base' of the quality indicators published by the Association of Dutch Health Insurers, that insurers planned to use for selective contracting and centralizing emergency care services, was fiercely disputed by medical specialists, hospitals and other stakeholders.

These incidents show that the justification and legitimation of difficult policy decisions with regard to the accessibility, efficiency and quality of health care are not always 'solved' by referring to 'evidence' (see also Syrett, 2003, 2013; Syrett, 2016). In the light of the growing recognition of the shortcomings of a technocratic approach, not least by the National Health Care Institute itself, this book investigates the epistemic practices that shape the knowledge-intensive decision-making processes of the Institute. Its point of departure is formed by the following research questions:

\section{How to understand the contemporary epistemic practices that shape the decision- making processes at the National Health Care Institute?}

- What are the preferred scientific methods, dominant notions of 'evidence' and epistemic ideals of 'objectivity' employed by the National Health Care Institute in its efforts to attain impartiality and justice in policy decisions?

\section{How can these epistemic practices be interpreted in terms of doing 'epistemic justice' in decision-making processes?}

- What types of knowledge are recognized as credible and intelligible contributions to the decision-making process?

- Which groups of knowers have (or lack) the interpretative resources to make themselves understood in the decision-making process?

Finding answers to these questions can ultimately help the National Health Care Institute to improve social inclusiveness, social robustness and representativeness in reaching difficult policy decisions. 
As such this study provides insight in how to stimulate the public legitimacy of decisions made in the context of social health insurance.

\section{METHOD}

Day-to-day policy practices in which science and politics intersect are the object of this book. It is in these practices that public accountability is 'done' by justifying and legitimating public policy choices by drawing on specific sources of knowledge and expertise. This study is based on ethnographic research into epistemic practices of the Dutch National Health Care Institute. As such, it offers an analysis of the Institute's 'machineries of knowing' (Knorr Cetina, 1999) - the working methods, techniques or tools of knowing - and examines how these practices constitute, not only epistemic ideals of 'objectivity', but also norms of public accountability.

As is customary in the field of STS, I refrain from taking any stance myself on what counts as 'evidence,' 'scientific' or 'objective' knowledge. The abstention from taking a standpoint allowed me to conduct a systematic research into the way others ascribe meaning, truthfulness, political significance and moral weight to particular forms of knowledge. What types of knowledge (e.g. experimental evidence, expertise, experience of doctors and patients) are used by the National Health Care Institute in its efforts to attain impartiality, objectivity and justice in policy decisions? What are the preferred scientific methods, dominant notions of 'evidence' and epistemic ideals of 'objectivity'?

The Institute's management provided me with an in-house desk (attended one to three days a week), and access to archives. During the four years of fieldwork, between October 2013 and October 2017, I had the opportunity to attend public and closed meetings of internal working groups (weekly), the Institute's Executive Board (two-weekly), as well as expert meetings of advising committees (Package Advisory Committee, Health Care Quality Committee, Scientific Advisory Committee, Health Care Professions Committee), staff fora and informal lunches. Empirical data were collected through direct observation, informal in-depth conversations, formal (semi-structured) interviews and multi-source documentary analysis. In meetings, fora and informal conversations I observed the 'practices of knowing' of those working at the Institute and recorded them in masses of detailed field notes.

Three real-life struggles of the National Health Care Institute in their decision-making processes emerged during the first year of fieldwork and my research focused progressively on these cases. First, there was the lawsuit on the reimbursement of bladder instillations for patients suffering from Interstitial Cystitis that was labelled by staff members at the Institute as the "hot potato" that worried many (notes\#18102013-24). I started to closely follow the lawsuit from the start of the legal proceedings in December 2013 till the judicial verdict in November 2014. A second flagship case for the Institute was when it used its 'overriding authority' for the first time with regard to quality standards. In 2013 the Institute was asked to lay down quality indicators for Emergency 
care, because private actors in the health care system failed to reach consensus about these quality metrics. I progressively focused my research into the Institute's work on quality management by following this trajectory. Later, in 2014, the Minister of Health asked the Institute to find new ways to improve the efficiency of public spending on the basic benefits package. Therefore, as a third case, I started to follow the coming into being of a new and promising approach to package management: a systematic screening of the basic benefits package to detect and eliminate overuse, underuse and misuse of health services covered from basic health insurance.

Each case was studied in the same consecutive stages: 1) familiarization with the case through informal conversations with staff and preliminary observations in meetings and fora; 2) supplementary data-gathering through multi-source documentary analysis (emails, minutes, internal and official documents, media articles) and selective (non-participant) observation of relevant meetings; and 3 ) in-depth research and triangulation through interviews with informationrich interviewees whom I selected through 'purposive' sampling (Green \& Thorogood, 2004). Formal interviews were transcribed in full and manually coded and analyzed. As a member-check, I sent written versions of the analyses of the different cases to interviewees and involved staff members in order to test my case-descriptions and analyses on them.

A four-year ethnographic research project is necessarily a process in which new insights develop alongside data analysis. It was an iterative process going from writing cumulative masses of field notes, studying relevant academic literature, selecting cases, returning to field notes, rereading theoretical studies, etc. This switching back and forth between theory and practice allowed me to gain a deeper understanding of the epistemic practices of the National Health Care Institute.

\section{OUTLINE OF THE BOOK}

The different chapters of this book each provide an in-depth empirical analysis of how the National Health Care Institute substantiates and justifies complex policy decisions, and reflect on the social representativeness and societal consequences of these epistemic practices.

Chapter 1 analyzes how the National Health Care Institute substantiated its decision against the reimbursement of bladder instillations with chondroitin sulphate or hyaluronic acid. In particular, the chapter discusses the ensuing lawsuit in which the Patients' Association for Interstitial Cystitis (a bladder condition) legally challenged the Institute for basing its decision primarily on scientific evidence from Randomized Controlled Trials (RCTs), thereby overruling clinical expertise and patients' experiences. The lawsuit centered on the question of whether the logic of EBM employed by the Institute, that emphasizes quantitative population-based research, is an adequate and fair method for making reimbursement decisions. Analyzing the court case in detail, this chapter engages with questions regarding the appropriateness and representativeness of the EBM-method in substantiating reimbursement decisions. 
Chapter 2 addresses the Institute's epistemic work in quality management. It discusses a dispute between insurers, hospitals and doctors about a set of quality indicators for emergency care. In 2013, private insurers formulated quality indicators drawing from clinical guidelines, RCTs and systematic reviews. Insurers planned to use the quality indicators to selectively contract emergency services and centralize Dutch emergency care in a few specialized hospitals. Insurers' plans caused much debate in the health care arena. Medical professionals questioned whether insurers' use of the indicators actually served to promote the quality of Dutch emergency care. Chapter 2 explores the work of the National Health Care Institute in mediating this dispute. It engages with questions regarding the adequacy, representativeness and appropriateness of quality metrics as a tool for allocating resources in the health care landscape, and addresses the role of EBM in this type of quality management.

Chapter 3 describes the epistemic work of the Institute in what was initially a 'disinvestment' initiative within the context of the Dutch basic benefits package. Faced with growing budget pressure, policymakers worldwide recognize the necessity of strategic disinvestment from ineffective, inefficient or harmful medical practices. Disinvestment programs, however, typically face substantial social, political and cultural challenges, such as mistrust, and struggles for clinical autonomy. Chapter 3 discusses the working method developed by the Institute to identify overuse, underuse and misuse of medical services currently covered from public funds. With this method the Institute, together with clinical experts and field parties, started comparing professional norms for clinical practice (as stated, for example, in clinical guidelines) with the care actually delivered and paid for in practice (for example, by looking at administrative data). Chapter 3 investigates how the Institute dealt with the socio-political sensitivities associated with disinvestment initiatives. It also engages with questions regarding the societal consequences of this type of knowledge work.

Chapter 4 returns to the cases discussed in chapters 1 and 2 and studies how epistemic practices in policymaking are entangled with questions of 'epistemic justice' (Fricker, 2007). Applying the idea of epistemic injustice to healthcare, some have argued that EBM is liable to generate epistemic injustice due to its focus on quantitative population-based research that privileges quantitative types of evidence and styles of articulating testimonies and - as a result - potentially undervalues the qualitative testimonies of doctors and patients. How can we interpret the epistemic practices in the context of reimbursement decisions (Chapter 1) and quality management (Chapter 2) in terms of 'epistemic (in)justice'?

The concluding chapter returns to the general research questions: What are the contemporary epistemic practices - the preferred scientific methods, dominant notions of evidence and epistemic ideals of 'objectivity' - of policymakers and experts working on the science-policy nexus in Dutch health care? How is public accountability for policy choices constituted in these practices? Looking at these epistemic practices from the perspective of 'epistemic injustice' (Fricker, 2007): what opportunities exist for improving the social inclusiveness and representativeness of these epistemic practices? 


\section{EXPERTS AND ‘IDIOTS’}

The bold choice for the title 'Experts and Idiots' demands an explanation up front. By choosing this title, I risk the possibility of a painful misunderstanding, especially as the book is based on ethnographic research. The last thing I would want is for readers to think that I would ascribe the qualification 'idiot' to anyone I have studied. In no way do the people who are the topic of this book deserve such a description. So, let me be clear: the term 'idiot' does not refer to any specific person.

Those who like their Russian classics may remember prince Lev Myshkin depicted by Dostojevski in his book 'The Idiot' (1869). Myshkin, who returned home after spending some years in a Swiss sanatorium, is portrayed as someone who does not seem to understand Russian societal values, conventions, assumptions and norms. The prince misses jokes and metaphors, embarrasses himself, asks bizarre questions and defends strange positions. Myshkin is a benign and childlike person who, with his confused presence, questions social norms and societal conventions. Dostojevski's 'idiot' is not a 'stupid person' or 'a person of low intelligence', as defined in modern dictionaries (Oxford English Dictionary, 2018). In ancient Greece, the term 'idiot' referred to someone who does not speak the language of the polis and was therefore cut off from civilized community (Farías, 2017).

Following Deleuze and Guattari, Stengers uses Dostojevski's idiot as a 'conceptual persona'4 who can help us rethink politics and decision-making in contemporary society (Stengers, 2005). For Stengers, the term 'idiot' refers to those chronic and complete nonconformists who do not want to be included; who want to talk about different things 'because there is something more important', who 'resist the consensual way in which the situation is presented' (Stengers, 2005, p. 994). Stengers' idiot is the one who slows down the process of achieving consensus because he denies the legitimacy of the political idiom (Watson, 2014, p. 89). Stengers' character of the idiot can serve as 'a heuristic for interrogating "what we are busy doing"', so that we do not get too comfortable with, for example, our policymaking methods or political routines (Michael, 2012, p. 529). Those who refuse to fit into the system re-open confusion, uncertainty and ambiguity. It is this confusion that we need in order to learn, innovate and reinvent, not least in the context of defining public problems and formulating policy solutions.

According to Michael, 'the idiot should be regarded not so much as a figure, but as a process or a sensibility that chronically seeks and engages this irritating absent-present other' (Michael, 2012, p. 544). The term 'idiots' in the title of this manuscript refers to Stengers' conceptual figure and Michael's process: the challenging lawsuits, the public controversies, the open protests of patients, the refusal of field parties, any non-conformist event. 'Idiots' are a prerequisite for reflection on our epistemic practices, our preferred scientific methods, our dominant notions of 'evidence' and our epistemic ideals of 'objectivity'. So that we do not get too comfortable with 'what we are busy

\footnotetext{
${ }^{4}$ Stengers borrows the idea of the 'conceptual personae' from Deleuze and Gauttari (chapter 3 Deleuze \& Guattari, 1999).
} 
Introduction

doing' (Michael, 2012). While experts have an obvious and widely recognized role in our democratic societies, idiots are of no less importance and deserve our explicit attention. Let us learn from experts and idiots alike. 


\section{REFERENCES}

Beckman, H., \& Frankel, R. (1984). The Effect of Physician Behavior on the Collection of Data. Annals of Internal Medicine, 101(5), 692-696.

Berg, M., \& Van der Grinten, T. (2003). The Netherlands. In C. Ham \& G. Robert (Eds.), Reasonable Rationing: International Experience of Priority Setting in Health Care (pp. 115-140). Maidenhead, Philadelphia: Open University Press.

Beyens, N., \& Bolt, T. (2017). 'A Medical Doctor in Politics'. Els Borst-Eilers and the Rise of Evidence-Based Healthcare in the Netherlands. BMGN-Low Countries Historical Review, 132(1), 16 v37.

Bijker, W., Bal, R., \& Hendriks, R. (2009). The paradox of scientific authority: the role of scientific advice in democracies. Cambridge, Mass.: MIT Press.

Bijlsma, M., Boone, J., \& Zwart, G. (2009). Selective Contracting and Foreclosure in Health Care Markets. CentER Discussion Paper Series No. 2009-89, TILEC Discussion Paper No. 2009-045.

Bolt, T. (2015). A doctor's order: the Dutch case of evidence-based medicine (1970-2015). AntwerpenApeldoorn: Garant.

Boon, W., Martins, L., \& Koopmanschap, M. (2015). Governance of conditional reimbursement practices in the Netherlands. Health Policy, 119(2), 180-185.

Boyce, T. (2007). Health, risk and news. New York: Peter Lang.

Campbell, S., Roland, M., \& Buetow, S. (2000). Defining quality of care. Social science \& medicine, 51(11), $1611-1625$.

Carel, H., \& Kidd, I. (2014). Epistemic injustice in healthcare: a philosophial analysis. Medicine, Health Care and Philosophy, 17(4), 529-540.

Coady, D. (2010). Two Concepts of Epistemic Injustice. Episteme, 7(2), 101-113.

Collins, H. (1985). Changing order: Replication and induction in scientific practice. London: Sage.

Daston, L., \& Galison, P. (1992). The image of objectivity: the talismanic image. Representations, 40(fall), 81-128.

de Jonge, H., \& Nijland, Y. (2018, 21 November 2018). 'Gegoochel met cijfers geluidsoverlast Lelystad Airport'. NOS. Retrieved from https://nos.nl/nieuwsuur/artikel/2260277-gegoochel-met-cijfers-geluidsoverlast-lelystadairport.html

de Zeeuw, H. (2017, 17 October 2017). Fouten in rapport naar geluidshinder van Lelystad Airport. NRC Handelsblad. Retrieved from https://www.nrc.nl/nieuws/2017/10/17/vliegverkeer-rekenfouten-in-rapport-naargeluidshinder-van-lelystad-airport-13533394-a1577540

Deleuze, G., \& Guattari, F. (1999). What is philosophy? London: Verso.

Delnoij, D., Rademakers, J., \& Groenewegen, P. (2010). The Dutch Consumer Quality Index: an example of stakeholder involvement in indicator development. BMC Health Services Research, 10(88).

Dieleman, S. (2015). Epistemic Justice and Democratic Legitimacy. Hypatia, 30(4), 794-810.

Djulbegovic, B., \& Guyatt, G. (2017). Progress in evidence-based medicine: a quarter century on. The Lancet, 390(10092), 415-423.

Eddy, D. (2005). Evidence-Based Medicine: A Unified Approach. Health Affairs, 24, 9.

Enthoven, A., \& Van de Ven, W. (2007). Going Dutch - Managed-competition health insurance in the Netherlands. The New England Journal of Medicine(357), 2421-2423. 
Evidence-Based Medicine Working Group. (1992). Evidence-based medicine. A new approach to teaching the practice of medicine. JAMA, 268(17), 2420-2425.

Farías, I. (2017). An Idiotic Catalyst: Accelerating the Slowing Down of Thinking and Action. Cultural Anthropology, 32(1), 35-41.

Felt, U., \& Fochler, M. (2010). Machineries for Making Publics: Inscribing and De-scribing Publics in Public Engagement. Minerva, 48(3), 219-238.

Fricker, M. (2007). Epistemic injustice: power and the ethics of knowing. Oxford: Oxford University Press.

Green, J., \& Thorogood, N. (2004). Qualitative Methods for Health Research. London: SAGE Publication.

Groene, O., Skau, J., \& Frølich, A. (2008). An international review of projects on hospital performance assessment. International Journal for Quality in Health Care, 20(3), 162-171.

Guyatt, G., Oxman, A., Vist, G., Kunz, R., Falck-Ytter, Y., Alonso-Coello, P., \& Schünemann, H. (2008). GRADE: an emerging consensus on rating quality of evidence and strength of recommendations. BMJ, 336(7650), 924-926.

Guyatt, G., Sackett, D., Sinclair, J., Hayward, R., Cook, D., \& Cook, R. (1995). Users' Guides to the Medical Literature: IX. A Method for Grading Health Care Recommendations. JAMA, 274(22), 1800-1804.

Hahn, H., Neurath, O., \& Carnap, R. (1929). Wissenschaftliche Weltauffassung: der Wiener Kreis (The Scientific Conception of the World: The Vienna Circle). In Ernst Mach Society (Ed.). Vienna.

Helderman, J., De Kruijf, J., Verheij, J., \& Van Thiel, S. (2014). Dike-Reeve of the health care polder. A politicalsociological analysis of the realisation of the National Health Care Institute against a backdrop of a changing policy agenda and changing political-administrative and societal relations. Diemen: Zorginstituut Nederland.

Helderman, J., Schut, F., van der Grinten, T., \& van de Ven, W. (2005). Market-oriented health care reforms and policy learning in the Netherlands. Journal of Health Politics, Policy and Law, 30(1-2).

Hemerijck, A. (1993). The historical contingencies of Dutch corporatism. University of Oxford, Oxford.

Hilarion, P., Suñol, R., Groene, O., Vallejo, P., Herrera, E., \& Saura, R. (2009). Making performance indicators work: The experience of using consensus indicators for external assessment of health and social services at regional level in Spain. Health Policy, 90(1), 94v103.

Hoffman, A. (2011). Talking Past Each Other? Cultural Framing of Skeptical and Convinced Logics in the Climate Change Debate. Organization \& Environment, 24(1), 3-33.

Hookway, C. (2010). Some Varieties of Epistemic Injustice: Reflections on Fricker. Episteme, 7(2), 151-163.

Hoppe, R. (1999). Policy analysis, science and politics: from speaking truth to power to making sense together. Science and Public Policy, 26(3), 201-210.

Hoppe, R. (2010). Lost in translation? A boundary work perspective on making climate change governable. In P. Driessen, P. Leroy, \& W. Van Vierssen (Eds.), From climate change to social change. Perspectives on science-policy interactions (pp. 107-130). Utrecht: International Books.

Horstman, K. (2001). Public bodies, private lives : the historical construction of life insurance, health risks, and citizenship in the Netherlands 1880-1920. Rotterdam: Erasmus Pub.

Horstman, K. (2014). Struggling with science and democracy. Public health and citizenship in the Netherlands. In F. Huisman \& H. Oosterhuis (Eds.), Health and Citizenship. Political Cultures of Health in Modern Europe (pp. 191-208). London: Pickering and Chattoo Publication.

Irwin, A. (2006). The Politics of Talk: Coming to Terms with the 'New' Scientific Governance. Social Studies of Science, 36(2), 299-320. 
Jasanoff, S. (1990). The fifth branch: Science advisers as policymakers. Cambridge, MA: Harvard University Press.

Kidd, I., \& Carel, H. (2017). Epistemic Injustice and Illness. Journal of Applied Philosophy, 34(2), 172-190.

Klintman, M. (2002). The Genetically Modified (GM) Food Labelling Controversy: Ideological and Epistemic Crossovers. Social Studies of Science, 32(1), 71-91.

Knorr Cetina, K. (1981). The Manufacture of Knowledge: An Essay on the Constructivist and Contextual Nature of Science. Oxford: Pergamon Press.

Knorr Cetina, K. (1999). Epistemic Cultures: How the Sciences Make Knowledge. Cambridge, Mass.: Harvard University Press.

Knorr Cetina, K. (2007). Culture in global knowledge societies: knowledge cultures and epistemic cultures. Interdisciplinary Science Reviews, 32(4), 361-375.

Latour, B. (1987). Science in Action: how to follow scientists and engineers through society. Cambridge, Mass.: Harvard University Press.

Latour, B., \& Woolgar, S. (1979). Laboratory Life: the construction of scientific facts. Princeton (N.J.): Princeton University Press.

Lindblom, C. (1959). The science of “Muddling Through". Public Administration Review, 19(2), 79-88.

Lövbrand, E. (2011). Co-producing European climate science and policy: a cautionary note on the making of useful knowledge. Science and Public Policy, 38(3), 225-236.

Lynch, M. (1985). Art and artifact in laboratory science : a study of shop work and shop talk in a research laboratory. Routledge \& Kegan Paul, London; Boston.

Michael, M. (2012). "What Are We Busy Doing?”: Engaging the Idiot. Science, Technology, \& Human Values, 37(5), 528v554.

Murphy, M. (2006). Sick building syndrome and the problem of uncertainty: environmental politics, technoscience, and women workers. Durham [N.C.]: Duke University Press.

Niezen-van der Zwet, M. (2012). Working towards legitimacy in decision-making: on governing appropriate medicine use and reimbursement in health care. Erasmus Universiteit, Rotterdam.

NOS. (2012, 21 September 2012). 'Ik ben te duur, dus ik krijg de doodstraf'. NOS journaal. Retrieved from https://www.nporadio1.nl/nos-journaal/onderwerpen/75585-ik-ben-te-duur-dus-ik-krijg-de-doodstraf

Oreskes, N. (2004). The Scientific Consensus on Climate Change. Science, 306(5702), 1686.

Oreskes, N., \& Conway, E. (2012). Merchants of Doubt: how a handful of scientists obscured the truth on issues from tobacco smoke to global warming. London: Bloomsbury.

Oxford English Dictionary. (Ed.) (2018). Online.

Parkhurst, J. (2017). The Politics of Evidence: From evidence-based policy to the good governance of evidence. New York: Routledge.

Partos, R. (2014). No immigrants, no evidence? The making of Conservative Party immigration policy. Political Insight, 5(3), 12-15.

Peeters, M., Delnoij, D., \& Friele, R. (2014). Stronger, but not (yet) an equal. The use of quality improvement instruments and strategies by patient organisations in the Netherlands. Social science \& medicine, 115, 56-63.

Popper, K. (1935 [1959]). Logik der Forschung [The Logic of scientific discovery]. London: Hutchinson and Co. 
Porter, T. (1994). Making things quantitative. SCIENCE IN CONTEXT, 7, 389-407.

Porter, T. (1995). Trust in Numbers: the Pursuit of Objectivity in Science and Public Life. Princeton: Princeton University Press.

Quine, W. V. (1951). Two dogmas of empiricism. New York: Longmans, Green \& Co.

Russell, J., \& Greenhalgh, T. (2009). Rhetoric, Evidence and Policymaking: a Case Study of Priority Setting in Primary Care (0068-1202). Retrieved from http://discovery.ucl.ac.uk/15560/1/15560.pdf

Sackett, D., Rosenberg, W., Muir Gray, J., Haynes, R., \& Richardson, W. (1996). Evidence based medicine: what it is and what it isn't. BMJ, 312(7023), 71-72.

Sanderson, I. (2002). Evaluation, Policy Learning and Evidence-Based Policy Making. Public Administration, $80(1), 1-22$.

Schinkelshoek, J., \& Martini, T. (2012). Meer dan een Incident: CVZ, Pompe \& Fabry. The Hague: Schinkelshoek \& Verhoog.

Staal, P., Heymans, J., Ligtenberg, G., Derksen, J., \& Couwenbergh, B. (2015). Beoordeling stand van de wetenschap en praktijk. Diemen: Zorginstituut Nederland.

Staal, P., Ligtenberg, G., \& van Drooge-van Loon, M. (2007). Beoordeling stand van de wetenschap en praktijk. Diemen: College voor Zorgverzekeringen.

Stengers, I. (2005). The cosmopolitical proposal. In B. Latour \& P. Weibel (Eds.), Making Things Public (pp. 9941003). Cambridge, Mass.: MIT Press.

Stone, D. (2002). Policy Paradox: the art of political decision making. New York: Norton.

Syrett, K. (2003). A Technocratic Fix to the "Legitimacy Problem"? The Blair Government and Health Care Rationing in the United Kingdom. Journal of Health Politics, Policy and Law, 28(4), 715-746.

Syrett, K. (2013). Courts, Expertise and resource allocation: Is there a judicial ‘legitimacy problem'? Public Health Ethics, 7(2), 112-122.

Syrett, K. (2016). Expanded HTA, Legitimacy and Independence: Comment on "Expanded HTA: Enhancing Fairness and Legitimacy." International Journal of Health Policy and Management, 5(9), 565-567.

Tanenbaum, S. (1995). Getting there from here: evidentiary quandaries of the US outcomes movement. Journal of Evaluation in Clinical Practice, 1(2), 97-103.

Tanenbaum, S. (2012). Improving the quality of medical care: the normativity of evidence-based performance standards. Theoretical medicine and bioethics, 33(4), 263-277.

Traweek, S. (1988). Beamtimes and Lifetimes: the world of high energy physicists. Cambridge, Mass.: Harvard University Press.

Van De Bovenkamp, H., Trappenburg, M., \& Grit, K. (2010). Patient participation in collective healthcare decision making: the Dutch model. Health Expectations, 13(1), 73-85.

Van de Ven, W., \& Schut, F. (2008). Universal mandatory health insurance in the Netherlands: A model for the United States? Health Affairs, 27(3), 771-781.

Van de Ven, W., \& Schut, F. (2009). Managed competition in the Netherlands: Still work-in-progress. Health Economics, 18(3), 253-255.

Van den Brink, R. (2014). Reimbursement of orphan drugs: the Pompe and Fabry case in the Netherlands. Orphanet Journal of Rare Diseases, 9(1), 017. 
Watson, M. (2014). Derrida, Stengers, Latour, and Subalternist Cosmopolitics. Theory, Culture \& Society Theory, Culture \& Society, 31(1), 75-98.

Weinel, M. (2008) Counterfeit Scientific Controversies in Science Policy Contexts. Cardiff University.

Weingart, P. (1999). Scientific expertise and political accountability: paradoxes of science in politics. Science and Public Policy, 26(3), 151-161.

Wood, M., Ferlie, E., \& Fitzgerald, L. (1998). Achieving clinical behaviour change: a case of becoming indeterminate. Social science \& medicine, 47(11), 1729-1738. 

Chapter 1

\section{Contested Evidence}

Based on: Moes, F., Houwaart, E., Delnoij, D. and Horstman, K. (2016)

'Contested evidence: a Dutch reimbursement decision taken to court', Health Economics, Policy and Law, 12(3), 325-344. 


\section{ABSTRACT}

This paper examines a remarkable lawsuit in health care rationing. The Patients Association for Interstitial Cystitis sued the Dutch National Health Care Institute for alleged misconduct against Interstitial Cystitis patients, as the Institute decided that bladder instillations with chondroitin sulphate or hyaluronic acid are no longer covered by the basic health insurance. The patients' organisation challenged the Institute for basing its standpoint on scientific evidence; overruling clinical expertise and patients' experiences. While scientific advice is often solicited in public health issues, simultaneously, the authority of scientific advice is increasingly being questioned in the public domain. Also, the judiciary is frequently called upon to adjudicate in rationing decisions. Based on an ethnographic study of the National Health Care Institute, drawing on insights from the field of Science and Technology Studies, we analyse this lawsuit as a negotiation of what knowledge counts in reimbursement decisions. 


\section{INTRODUCTION}

The Netherlands was recently host to a remarkable lawsuit in health care. The Patients' Association for Interstitial Cystitis (ICP) sued the health care rationing body of the Netherlands, the National Health Care Institute (hereafter 'the Institute'), for alleged misconduct against interstitial cystitis patients. In 2013, the Institute issued its authoritative standpoint that bladder instillations with chondroitin sulphate or hyaluronic acid [see Box 1] should no longer be covered by the basic health insurance. Despite the fact that patients claimed to benefit from the treatment, the Institute concluded that there was insufficient scientific evidence supporting its effectiveness. The patients' organisation legally challenged the Institute for basing its standpoint on scientific evidence, overruling clinical expertise and patient experiences.

\section{Box 1 | Bladder instillations}

Bladder instillations are a medical treatment used for various bladder conditions, e.g. bladder cancer, interstitial cystitis, painful bladder syndrome. Bladder instillations are a common procedure for urologists and patients can even do the procedure independently in a home setting. During treatment a fine tube (catheter) is inserted into the bladder. The bladder is then filled with a solution, including a pharmacological cocktail of e.g. chondroitin sulphate or hyaluronic acid. The solution remains inside for a period of time (30 minutes to two hours) and is then drained.

In court, the Institute's use of the principles of evidence-based medicine (EBM) was disputed. The singularity of this case lies in the fact that such a 'battle of the evidences' is the exact opposite of what the founders of EBM had in mind. According to David Sackett, the aim of EBM is to 'integrate the best external evidence with individual clinical expertise and patients' choice' (Sackett, Rosenberg, Muir Gray, Haynes, \& Richardson, 1996, p. 72 emphasis added). The Institute's evidence-based decision had quite the reverse effect; it led to a momentous clash between experimental and experiential forms of knowledge.

Patients, urologists and the Institute ended up in the courtroom of Amsterdam negotiating the appropriateness of different forms of knowledge for this decision. Drawing on insights of the field of Science and Technology Studies (STS), we studied the legal proceedings as a collective deliberation of what type of knowledge counts in reimbursement decisions. In court, what meaning is given to the concepts 'evidence', 'expertise' and 'experience'? What evidential weight is attributed to these respective forms of knowledge? Why were the Institute's principles of EBM contested in this lawsuit?

This lawsuit is not an isolated case. Over the past decades, the judiciary has increasingly been called upon to adjudicate in rationing decisions (Syrett, 2013). Increased litigation has been 
attributed to a waning deference to medical judgement, more explicit rationing strategies, a more rights-conscious citizenry and the rise of patient support organisations (Syrett, 2013, p. 112). Health care rationing institutes find themselves on shifting ground. They have to come to terms with the declining authority of science, a growing recognition and involvement of patients in policy decisions (Moreira, 2011; Wait \& Nolte, 2006) and increased judiciary interference (Morales, 2015; Syrett, 2013; Wang, 2013). These are delicate issues for knowledge institutions whose authority, legitimacy and very raison d'être lay, originally, in their acknowledged expertise as a basis for policy decisions. With this analysis, we aim to gain insight into the way in which court cases help rationing bodies like the National Health Care Institute to reflect on their epistemic conventions and - ultimately - to work towards more socially robust rationing decisions.

\section{THEORISING KNOWLEDGE DISPUTES}

Health care rationing decisions frequently trigger heated discussions in the media and politics. It is commonly assumed that scientific evidence provides the factual, medical-technical and nonpolitical base for such thorny, moral decisions. Yet, STS scholars have shown that there is no given distinction between fact and value (e.g. Latour, 1993) or between objective knowledge and subjective ideas (e.g. Daston \& Galison, 1992). Rather, such distinctions are 'man-made'. What we currently accept as valid knowledge claim results from a collective human endeavour that is contingent to our political and socio-historical setting.

In contemporary knowledge societies, scientific knowledge has become an integral element of politics and governance. At the same time, the authority of scientific advice is being challenged more frequently in the public domain (Bijker, Bal, \& Hendriks, 2009; Horstman, 2014). As knowledge has come to play a more central part in health care governance, the question of whose knowledge counts for such decisions seems to have become politically and morally charged (cf. Moreira, 2012). Some scholars have found that the processes of scientification of public policy often go together with processes of politicisation of scientific knowledge (Hoppe, 1999; Weingart, 1999). We regard the lawsuit on bladder instillations as one of those politicisations of scientific knowledge.

\section{METHODS}

This paper is the product of ongoing ethnographic research into 'how knowing is done' (de Laet, 2012, p. 429) being carried out at the National Health Care Institute, a central governing body in Dutch health care. It is within this research context that bladder instillations emerged as a prominent case, exemplary of the Institute's 'real life' struggles. Knorr Cetina introduced the notion of 'epistemic culture' that refers to the historically, politically and socially grounded practices of creating and warranting knowledge (Knorr Cetina, 1999, 2007). Every place that produces knowledge claims, be 
it a laboratory or a knowledge institute, is host to a set of 'practices, arrangements and mechanisms' which - in that area of professional expertise - make up 'how we know what we know' (Knorr Cetina, 2007, p. 363). The Institute too is governed by such knowledge routines.

The first author collected empirical data through direct observation, in-depth conversations and document analysis between October 2013 and October 2015. The Institute's management provided her with an in-house desk (attended one to three days a week), and access to archives. During two years of fieldwork, the first author attended public and closed meetings of internal working groups (weekly), the Institute's Executive Board (two-weekly), as well as expert meetings of advising committees (Package Advisory Committee, Health Care Quality Committee, Scientific Advisory Committee, Health Care Professions Committee), staff fora and informal lunches. She recorded the 'practices of knowing' at the Institute in detailed field notes: what types of studies or expertise are selected in decision-making processes? What preferences exist with regard to methods or disciplines? What are the shared beliefs regarding what a credible knowledge claim looks like?

A few days into the fieldwork, on 18 October 2013, a staff member with many years' experience referred to the issue of bladder instillations as "a hot potato" (notes\#18102013-24). Following this lead, the first author familiarised herself with the case in five in-depth conversations with the staff members involved. With the lawsuit pending, these conversations were replete with references to documents: research papers, journal articles, policy documents and procedural manuals in support of the Institute's standpoint. At least as many references were made to the letters of complaint, studies, expert opinions and testimonials brought forward by the plaintiff, the ICP. Necessarily, we delved into the Institute's archives to retrieve all documents on the case and searched PubMed for the journal articles and medical guidelines cited. The following response to a request for an interview illustrates the informational weight these documents had for staff members: what do you want? "just a little chat about it? [or] the documents?" (notes\#28102013). Legal records, policy and research papers, internal emails, memos, letters and testimonials were crucial informants in understanding the specificities of the case. Documents did indeed prove to be the prominent 'artefacts' of modern knowledge practices (Riles, 2006). In this paper, we cite many such documents, as they possess the conciseness necessary to depict this knowledge dispute satisfactorily.

We closely followed the lawsuit from the writ of summons in summary proceedings on 4 December 2013, until the judicial verdict in proceedings on merits on 19 November 2014. The staff members involved (both policy advisors and legal staff) kept us informed and we carried on tracking the paper trail and attended board meetings and meetings of advisory committees addressing the issue. Two years of ethnographic fieldwork permitted not only a close study of the case, but also of its impact on the Institute: the worries, doubts and reflexivity it brought with it. Being present enabled us to observe the Institute's continuing struggle to reconcile the unambiguous formal rationality of EBM with the 'messiness' of case-based practical rationality (Gkeredakis, Swan, Nicolini, \& Scarbrough, 2011; Jenkings \& Barber, 2004, p. 1765; Russell \& Greenhalgh, 2012, 2014) and the inevitable lack of evidence they often have to grapple with in making rationing decisions. 
All authors engaged in an iterative process of (joint) close reading of field notes and legal documents. Sensitised by the literature on EBM and its commentaries (Booth, 2010; Greenhalgh, Howick, \& Maskrey, 2014; Greenhalgh, Snow, Ryan, Rees, \& Salisbury, 2015; Parkhurst \& Abeysinghe, 2014; Petticrew \& Roberts, 2003; Sackett et al., 1996), we progressively focussed on three different forms of knowledge: experimental evidence, clinical expertise and patient experience. We culled from the data any written or verbal statement on the truthfulness, usefulness and significance of these forms of knowledge. In our analysis of the legal proceedings, we focussed on the deliberations of weighing up these respective forms of knowledge against one another. As a 'member-check', we discussed our analysis in staff fora, a general board meeting, and with a research team at the Institute.

Working from an STS perspective, we abstained from making any claims about the truthfulness, usefulness or political significance of particular forms of knowledge. Similarly, we make no claims about the appropriateness of the principles of EBM for rationing decisions. It is only by refraining from taking a stand of our own that we can conduct a systematic research into the way other parties give meaning to the notions of 'evidence', 'expertise' or 'experience'. By studying how others weigh these respective forms of knowledge and evaluate the appropriateness of the principles of EBM, we aim to learn what is currently required in substantiating and legitimating rationing decisions.

\section{TOWARDS EVIDENCE-BASED HEALTH CARE RATIONING (1965-2006)}

The Netherlands has standard basic health insurance for all citizens provided by private health insurance companies. The 2006 Health Insurance Act (Zorgverzekeringswet, Zvw) obliges everybody who resides - or pays payroll tax - in the Netherlands to take out basic health care insurance from a private insurance company. These private insurance companies are expected to negotiate the prices, services, and quality of care on behalf of their insured clients (Van de Ven \& Schut, 2008, p. 779). The market is heavily regulated (Leiber, Greß, \& Heinemann, 2015). Incomerelated subsidies make basic health care insurance affordable for all citizens. A risk adjustment scheme compensates insurance companies for clients with predictably high medical expenses (Van de Ven \& Schut, 2009). Benefits covered from basic insurance policy are broadly indicated by law ( $Z v w$ art. 10). The Minister of Health formally requests advice from the Institute to specify the medical-technical content of care that can be reimbursed from the basic health insurance. The Institute is asked to take a standpoint in cases lacking clarity about the reimbursement of specific treatments or where there is discord in the field. These standpoints are authoritative in the field. It is in this capacity that the Institute published a negative standpoint in 2013 , stating that bladder 
instillations with chondroitin sulphate or hyaluronic acid were not eligible for reimbursement from the basic health insurance (College voor Zorgverzekeringen, 2013a). ${ }^{1}$

In reaching this reimbursement decision, the Institute applied the principles of EBM. The way reimbursement decisions are made in the Netherlands developed over time. As long ago as 1965, the Dutch Sickness Funds Act (Ziekenfondswet, Zfw) determined that, for a medical treatment to be reimbursed from public funds, it must be considered 'customary' by the medical professional community:

'care provided by medical specialists, as referred to in article 8, para. 1, under a, of the Sickness Fund Act, includes medical, surgical and obstetric care that is determined, in scope, by what is common practice in the professional community' (Benefits in Kind (Sickness Fund) Decree, art.8, emphasis added)

From the late 1970s, this so-called 'common practice criterion' was much debated within the context of Dutch health care legislation (de Groot, 2006; Van Donk \& Tusschenbroek, 2005). Over a period of four decades, the 1965-criterion was reinterpreted and reformed by jurisprudence of the Central Appeals Tribunal (CRvB) and the European Court of Justice (ECJ). First, in the 1979 Acupuncture arrest, the CRvB ruled that 'common practice' should be interpreted as the 'attitude within the profession of medical science and medical practice' (21 December 1979). Later, the ECJ further developed the interpretation of 'common practice' in its 2001 ruling in the Smits-Peerbooms arrest. The court decided that 'common practice' could not be interpreted as 'current perceptions among Dutch professionals', but as care which 'has been deemed sufficiently tried and tested in the international world of medical science' (ECJ 2001, C-157/99). When the Health Insurance Act was implemented in 2006, the much criticised criterion of 'common practice' was replaced by a criterion that covered the overtones of the CRvB and ECJ jurisprudence:

'The content and range of the forms of care or health services are in part determined by established medical science and medical practice and, where there is no such criterion, by what is regarded in the relevant professional field as responsible and adequate care and services' (Health Insurance Decree (Besluit Zorgverzekeringen, Bzv art. 2.1, emphasis added))

This new criterion was an answer to national and European litigation, and to a general call for EBM in the health care sector itself. Legal staff at the Institute had interpreted the ECJ's 2001 ruling as follows: 'the court of appeal seems to be referring here to what is actually known as "evidencebased medicine"' (internal memo 2006).

\footnotetext{
${ }^{1}$ As of 1 April 2014, the Dutch Health Care Insurance Board (College voor Zorgverzekeringen, CVZ), became the National Health Care Institute (Zorginstituut Nederland).
} 
Starting in the 1970s, EBM gained strong traction in clinical practice and policy rooms, amid growing recognition of 'the vagaries of clinical experience' (Armstrong, 2002, p. 1772) and the inadequacies of knowledge based on personal experience alone. EBM emphasised the use of external, experimental evidence in medical decision making. The randomised-controlled trial (RCT) was seen as the 'gold standard' for determining the efficacy of a treatment: evaluating the impact of medical interventions on groups rather than on individuals and through controlled, experimental rather than observational design. The 2006 criterion shows that, in reimbursement policy too, there was a move away from confidence in knowledge based on doctor-patient interactions and their 'common treatment practices' (art.8, Sickness Fund Act) and a move towards the new evidencebased paradigm.

Since the introduction of the 2006 Health Insurance Act, the Institute has used the principles of EBM 'in order to determine whether care complies with the established medical science and medical practice criterion' (College voor Zorgverzekeringen, 2007). The EBM method currently functions as a tool for validating and weighing medical knowledge from both medical practice and science. It does this, by placing different forms of medical knowledge in a hierarchy²:

'A1) systematic review of at least two A2 level studies carried out independently of one another;

A2) good quality and sufficiently large randomised double-blind, comparative clinical research (RCT);

B) comparative research, but without all the characteristics of A2;

C) non-comparative research;

D) 'experts' opinions' (College voor Zorgverzekeringen, 2007, pp. 8-9)

The status of evidence within this hierarchy is based on the level of rigorousness in study design to reduce susceptibility to bias. The Institute selects medical-scientific information (scientific articles, medical guidelines, etc.) and ranks it according to its evidential value (level of evidence A1 to D). The 'cardinal principle of Evidence-based Medicine is that strong evidence supersedes weaker evidence' (College voor Zorgverzekeringen, 2007, pp. 8-9). The RCT and the systematic review form the apex of this hierarchical order. For positive reimbursement advice, the Institute requires at least one systematic review (A1-level) or two RCTs (A2-level) that show unambiguously positive results about the therapeutic effect of the treatment. As systematic reviews and RCTs are not always available or feasible, the Institute may accept lower levels of evidence in decision making. This may be the case if (double-) blind research is impossible, if the disorder is very rare, or if starting an

\footnotetext{
${ }^{2}$ Following national consensus on evidence-based guidelines, the Institute adopted the EBRO hierarchy of evidence (Evidence-Based Guidelines developed by the Institute for Health Care Improvement (CBO)). Similar hierarchies are used by affiliated health care rationing institutes, such as the British National Institute for Health and Care Excellence (NICE).
} 
$\mathrm{RCT}$ is no longer viable (because the treatment is a long-standing practice or endorsed by strong international consensus).

The EBM method offers the Institute a general procedure for weighing different forms of evidence and negotiating compatibilities between them. According to the Institute, the EBM method facilitates the 'meticulous, explicit and judicious use of the current best evidence' for reimbursement decisions (College voor Zorgverzekeringen, 2007). From an STS perspective, we could say that the EBM hierarchy provides the Institute with a 'logic of knowledge consumption' (Knorr Cetina, 2007, p. 368). The Institute implemented EBM as -to use the phrase coined by Knorr Cetina'epistemic machinery' (Knorr Cetina, 1999): a working method, technique or tool of knowing. This does not mean that the formal rationality of EBM is always this clear-cut when used in the practice of decision making. As a staff member commented:

"The difficulty [is] that often no good RCTs are available. You often have to be satisfied with 'lower evidence.' There is a 'check-list of appropriate evidence' [...] it lists all the reasons for diverging from higher evidence. But every case is still difficult. Every case is different and presents its own problems" (notes\#31102013)

In the case of bladder instillations, the staff members involved actually thought "last year we were starting on a clear-cut case, but it turned out to be fairly complicated" (notes\#31102013).

\section{CONTESTED EVIDENCE}

In 2011, increase in the price of a specific brand of bladder instillations (Uracyst) attracted the attention of insurance companies. Confronted with increased costs, insurance companies started asking questions about the added value of bladder instillations with chondroitin sulphate or hyaluronic acid $(0.9 \% \mathrm{NaCL})$. The Institute was asked for a standpoint on the issue. The Institute's decision-making process started with a literature search. They searched for experimental evidence from double-blind and placebo-controlled RCTs. This is why:

'As interstitial cystitis is a disorder that is characterised by a fluctuating course and there is no standard treatment, randomised studies are needed in which bladder instillation with washes containing chondroitin sulphate and/or hyaluronic acid is compared with placebo washes. As the symptoms and outcome parameters are subjective, doubleblind studies are preferred' (College voor Zorgverzekeringen, 2013a, p. 10).

Double-blind and placebo-controlled RCTs keep both patient and doctor in the dark about the treatment received. In EBM, it is generally felt that such an experimental design curtails a possibly biased cognition and facilitates a more 'objective' measurement of the treatment effect. 
The Institute consulted two databases, Cochrane Library and MEDLINE, specialised in biomedical literature, RCTs and systematic reviews. Two double-blind RCTs (A2-level evidence) were selected and a prospective comparative study (B-level evidence). The selected RCTs (Nickel, Egerdie, Steinhoff, Palmer, \& Hanno, 2010; Nickel, Hanno, Kumar, \& Thomas, 2012) showed improvements after treatment with bladder instillations with chondroitin sulphate or hyaluronic acid, but the difference with the placebo group was not statistically significant. The prospective comparative study (Shao, Shen, Rui, \& Zhou, 2010) showed statistically significant improvements (in micturition, Visual Analogue Scale (VAS) pain score and bladder capacity), but these were not statistically tested between the groups and the study was of low quality (College voor Zorgverzekeringen, 2013a, p. 11). The Institute also consulted international guidelines and reimbursement standpoints. In four international guidelines and an (outdated) standpoint of the Canadian Agency for Drugs and Technologies in Health, treatments with bladder instillations were either not mentioned, or had a low level of recommendation due to inconclusive evidence (College voor Zorgverzekeringen, 2013a, p. 13). From these findings, the Institute concluded that there was insufficient evidence of the effect of this treatment to arrive at a positive reimbursement standpoint.

Before publication, the Institute invited the Dutch Urological Association (NVU) to comment on the findings. The NVU did not contest that 'the outcomes [...] show that at the moment there is insufficient reliable research that provides evidence of the effect of the said washes in the indication fields mentioned' (excerpt from email). The NVU did, however, express some doubt about the conclusiveness of the experimental evidence:

'By the way, this does NOT prove that the washes are NOT effective, which can be confirmed, based on the experience of urologists who frequently use the said washes' (College voor Zorgverzekeringen, 2013a, p. 21).

The Institute, however, required positive proof of efficacy in order to include a treatment in the basic package: "care must above all be effective. This demands evidence showing that care does what it is meant to do' (Zorginstituut Nederland, 2014). Note that this requirement conflates situations where there is definitive evidence of no treatment effect, with situations where there is no conclusive evidence of a treatment effect. The only way towards a positive reimbursement decision would be conclusive proof of the treatment's effectiveness. That is why the Institute - despite the NVU's reservations - had to conclude that bladder instillations with chondroitin sulphate or hyaluronic acid did not fulfil the 'established medical science and medical practice' criterion as 'the existing evidence is insufficient for attributing a possible effect of using intravesical treatment with chondroitin sulphate and/or hyaluronic acid to treat interstitial cystitis' (College voor Zorgverzekeringen, 2013a, p. 19). One staff member noted: 'if new positive studies are published, [the Institute] can revise their standpoint' (email\#16122013). But for the time being the matter had been settled. In July 2013 the Institute published its standpoint that bladder instillations with chondroitin sulphate and/or hyaluronic acid would no longer be covered by the basic health insurance. 
Soon after this, the Dutch Urological Association (NVU) and the Patients' Association for Interstitial Cystitis (ICP) took up battle positions against the Institute. First, in a letter to the Institute, the NVU held an earnest plea for continued reimbursement of the instillations. The NVU mentioned the tremendous diversity in the manifestation of interstitial cystitis (IC), the groups of patients, the pathophysiology and the diagnostic findings. Different treatment plans are available for IC (e.g. bladder instillations, antibiotics, painkillers, neuroleptics, neuromodulation, botuine-toxine-A injections and cystectomy with urine deviation), and yet 'the various different complaints relating to interstitial cystitis are extremely persistent and difficult to treat' (letter from the NVU). The NVU argued that:

'In practice of state-of-the-art clinics that treat patients with IC, this means it is not possible to produce such hard scientific evidence to show which treatments work and which do not. Large series are impossible due to the diversity of the groups of patients and the way in which IC presents, which means that hard evidence will never be supplied. These patients need customised treatment and in the opinion of the experts these bladder fluids are indispensable' (letter from the NVU).

Can this complex disorder be adequately captured by an experimental research design? Can the results of the two RCTs (with sample populations of respectively 65 and 98 people) be projected onto the entire population of IC patients? The Institute acknowledged the difficulty of the issue. One of the scientific advisors to the Institute would later put it as follows: "It is increasingly difficult to be able to say in general whether something does or doesn't work... It depends increasingly on how well you define the target group" (notes\#10022014). Together, the NVU and the Institute decided that 'the best option would be to attempt to identify, based on the existing studies, a subgroup that would benefit from the instillations' (email\#14012014). On the basis of such a subgroup analysis, the Institute may decide to reimburse the washes for specific groups of IC patients, for example patients that show Hunner's lesions. However, after reviewing the issue, the Institute's staff concluded that 'based on the literature [...], there is a vague idea that a subgroup exists, but identifying the subgroup is still not possible' (email\#14012014). In the end, it was left to the NVU to come up with a guideline and specified treatment protocol for (different groups of) IC patients. The Institute suggested that, if such a guideline contained an 'extremely accurate description of the indication and the expertise required of the urologist', the Institute might decide that 'no RCTs will be necessary for such a limited group, as we can regard a lower level of evidence as acceptable' (email\#140102014). To this day, however, such a guideline has not been made.

The ICP, too, wrote a letter to the Institute in which it lodged a notice of objection. 'You were wrong', the ICP wrote to the Institute, 'not to take into account in the report - or insufficiently - the opinion of the professional group, which is that this is a case of responsible and adequate care' (letter from the ICP). This issue was deliberated in court (we will address this issue later). The letter also included several testimonials in which patients claimed that bladder instillations with 
chondroitin sulphate or hyaluronic acid were less invasive and less painful compared to regular washes without this pharmaceutical cocktail. One of the testimonials read:

'The effects of GEPAN can clearly be observed. After bladder instillation washes, the frequency of urination is considerably reduced and nocturnal frequency is also reduced. Furthermore, and naturally because of this, one can also speak of a greatly reduced urge and pain' (exhibit 17).

Though the Institute never actually denied that individual IC patients might benefit from a treatment with bladder instillations, they did not take patient testimonials into account. A staff member explained:

'The fact that individual patients claim to benefit from the treatment is not sufficient to conclude that the treatment complies with 'established medical science and medical practice.' [The Institute] does not issue statements about and on behalf of individual patients' (email\#16122013).

The Institute needed RCTs. Apart from the testimonials, the ICP presented new publications from 2013 showing that patients benefitted from washes with chondroitin sulphate and/or hyaluronic acid. The Institute did decide to review those publications. In an updated standpoint, published at the end of November 2013, they wrote:

'Due to a letter of objection from the Patients' Association for Interstitual Cystitis, a supplementary literature search was carried out for studies dated from November 2012 up to and incl. 27 November 2013. No new randomised studies were found' (College voor Zorgverzekeringen, 2013b, p. 11).

The Institute did find a meta-analysis of a non-comparative study by (Nickel et al., 2009) and the two earlier mentioned RCTs by (Nickel et al., 2010; Nickel et al., 2012) in which: the 'pooling of these 3 studies revealed that bladder instillation with chondroitin sulphate for interstitial cystitis is effective' (College voor Zorgverzekeringen, 2013b, p. 9). However, the Institute concluded that 'the way this meta-analysis was carried out was methodologically incorrect, because the studies differ from the study set-up, which resulted in a distortion of the results' (College voor Zorgverzekeringen, 2013b, p. 11). So the updated standpoint conveyed the same message as before: that the existing evidence was insufficient for attributing a possible effect to bladder instillations with chondroitin sulphate and/or hyaluronic acid and the washes would no longer be covered by the basic health insurance. Staff at the Institute recalled that: 
'the studies and patient case histories supplied by the ICP did not form a reason for [the Institute] to alter its current standpoint. We confirmed this on 26 November 2013 in a letter to the ICP. [...] On 4 December the ICP summoned [the Institute] to appear in summary proceedings' (internal memo\#07012014).

The legal proceedings started with summary proceedings (ECLI:NL:RBAMS:2014:66) and were followed by proceedings on the merits (ECLI:NL:RBAMS:2014:7727).

\section{LEGAL PROCEEDINGS}

The lawyer of the ICP stated before the court:

"I do not contradict that the efficacy of a treatment is a prerequisite for being eligible for inclusion in the insured benefits package. I only contradict that this efficacy has to be proved according to the EBM method [...], I feel that the element of 'practice' is insufficiently accounted for in the EBM method [...] What everyday practice thinks about something has too little evidential value in the EBM method" (hearing\#30042014)

According to the lawyer of the ICP, clinical practice is given too little weight in the EBM method and the Institute's standpoint - wrongfully - relied predominantly on RCT-level evidence. The ICP presented a declaration signed by 84 urologists (of the 432 urologists registered with the Royal Dutch Medical Association (KNMG) at that time), claiming that consensus exists amongst urologists that the bladder instillations are effective for some patients. She referred to an article by the founders of EBM (stressing that external evidence alone is never enough, nor is solely clinical expertise, both are essential for medical decision making (Sackett et al., 1996) to claim that knowledge from "everyday medical practice should be placed on the same level as other forms of evidence" (hearing\#30042014).

In response to the urologists' declaration, the Institute argued that in this case 2 RCTs were available; 'at such time that such scientific literature is available, the opinions of experts are in fact no longer relevant (strong evidence replaces weaker evidence)' (email\#17122013). The chairman of the Institute's Scientific Advice Committee explained further: "The EBM method uses not only RCTs [...]; this form of research is required in particular for those treatments of which we have reasonable doubt about their efficacy" (hearing\#30042014). In the case of bladder instillations, there was such a considerable doubt. A staff member explained: 
"Some of the patients have a functional syndrome... and these are subjective outcome measures that you examine, i.e., improvement or reduction in symptoms. We were therefore explicit in saying "we want randomised studies and not case series because ... the placebo group also had a large response"' (notes\#03032014).

Weighing the evidential value of clinical expertise against that of RCTs, the judge was of the opinion that the Institute provided grounds showing that, in this case, there was indeed 'a need of double-blind, controlled research in order to objectively substantiate the experience of doctors' (ECLI:NL:RBAMS:2014:7727). Hence, in the judicial verdict on 19 November 2014, the court found it was:

'justified that, despite the importance of practice experience of doctors - which is acknowledged by the Institute - this is of less consequence than the results of doubleblind, controlled studies' (ECLI:NL:RBAMS:2014:7727, emphasis added).

The two RCTs by (Nickel et al., 2010; Nickel et al., 2012), however, did not remain undisputed in court. In the original standpoint, the Institute stated that: 'both studies included patients numbers of, respectively 65 and 98 patients, and were under-powered. The duration of follow-up was short' (College voor Zorgverzekeringen, 2013a, p. 15). In the course of the lawsuit, the Institute invited an independent research institute (ME-TA) to review the literature using a new EBM method: Grading of Recommendations Assessment, Development and Evaluation (GRADE). GRADE is presented as an innovative EBM method in which the hierarchy of evidence is maintained, yet evidence can be upgraded or downgraded according to its (methodological) quality. The method increases the flexibility of the traditional hierarchy. The GRADE method is regarded as a transparent, explicit and systematic method for weighing evidence not only according to the rigorousness of the study design, but also the quality of that evidence (e.g. Guyatt et al., 2011). ME-TA's application of the GRADE method to the literature led to the downgrading of RCTs due to quality considerations (underpowered, inadequate duration of follow-up). However, none of the other forms of evidence were upgraded. Working from the cardinal principle that strong evidence supersedes weaker evidence, the RCTs still superseded other forms of knowledge available. The GRADE method did not alter the Institute's conclusions. The judicial verdict read:

'The conclusion of this [GRADE] study is also that the existing evidence is insufficient to be able to attribute a possible effect to the treatment with these bladder instillation products [...] Therefore, insofar as it could already be said that the Institute did not make use of the most advanced method for assessing care against [established medical science and medical practice] to arrive at its [initial] standpoint on 23 July 2013, this may remain without consequences, as the newer method did not lead to any different result' (ECLI:NL:RBAMS:2014:7727). 
After all, the evidential value of RCTs - despite serious quality considerations - was considered to be higher than the evidential value of clinical expertise.

In what follows, we focus on deliberations weighing patient experiences against RCTs. In court, the ICP reproached the Institute for failing to consult patients' organisations in its standpoint. The ICP stated that it "was never involved in the Institute's research. We were only informed after the standpoint had already been determined" (hearing\#30042014). According to the ICP, this was a breach with the 'due care principle' in policymaking. In its defence, the Institute claimed that it is standard procedure to consult professional associations of medical specialists (in this case the NVU), but not necessarily patients' organisations. The Institute takes into account the 'specific circumstances and interests of the group of patients' only when there are questions about the feasibility of scientific research or if 'it is necessary to be satisfied with a lower level of 'evidence'. This is [...] not the case here' (statement of defence). The Institute explained that in this case 'two RCTs had been carried out, proving that it is possible to carry out a double-blind RCT involving this disorder' (statement of defence). As RCTs were available, they took precedence over patient preferences and testimonials for this reimbursement decision. The Institute claimed:

'specifically because this involves a heterogeneous group of patients, it is necessary to carry out research among subgroup of patients in order to demonstrate that the treatment is effective for specific groups of patients. The Institute has always maintained that, when such research becomes available, a new assessment can take place that may result in a different opinion' (statement of defence).

During the legal proceedings, the ICP presented journal articles and abstracts of promising studies. It made no difference, however, as none of these studies matched the evidential power that was attributed to the two RCTs used in the Institute's standpoint. All in all, the judge ruled that:

'In the opinion of the court, it is irrelevant whether failing to consult the patients' association is incompatible with the due care principle, as it has been established that the information that the ICP could have supplied - and which has been supplied during these proceedings - did not lead to a different assessment' (ECLI:NL:RBAMS:2014:7727, emphasis added).

Furthermore, the ICP noted that "no attention whatsoever was given to 'soft principles', such as quality of life, patient satisfaction and the experience of patients and care providers" (hearing\#30042014). According to the Institute, however, individual patient preferences do not play any significant role in collective coverage decisions (College voor Zorgverzekeringen, 2007, p. 10). A director at the Institute pointed out the reason why RCTs are valued over patient preferences: 
"the use of non-personal statistical data on a group level makes it possible to weigh up the personal interests of one group of patients against those of another group of patients. Every euro that you spend on bladder instillation liquids is no longer available for spending on care for elderly persons suffering from dementia, to name just one..." (notes\#25062015)

For health economic purposes, RCTs permit a meta-assessment of health benefits between different treatments. The use of RCTs and systematic reviews enables one to calculate the expected gain from different treatments, thus facilitating a choosing between them. One of the board members explained that the Institute always has to consider the limited public funds available and is tasked to ensure "that the general public, who pay the premiums, are paying for something that is worthwhile" (notes\#30062015). Without evidence of the effectiveness of a treatment, the government could be paying for care that is actually ineffective or - in a worst case scenario - is facilitating care that might be doing more harm than good. In the end, the court decided, based on the same line of reasoning, that:

'It is the intention of the legislator to confine the basic benefits package to those forms of care that can be trusted to be effective. This is to be tested against 'established medical science and medical practice.' Whether care satisfies this legal criterion must be determined as objectively as possible. In the opinion of the court, the Institute has given - with its selected method [EBM] - a correct application of this criterion' (ECLI:NL:RBAMS:2014:7727)

\section{DISCUSSION}

In this article, we showed how and why the Institute reached a negative reimbursement decision with regard to bladder instillations with chondroitin sulphate or hyaluronic acid for the treatment of IC. Our analysis of the decision-making process showed the tension between EBM's formal logic and its practical rationality: the struggle to be accountable to both 'good' EBM and local contingencies (Jenkings \& Barber, 2004, p. 1765). The opinions of clinical experts and patients' experiences with the treatment, though positive, were considered unreliable due to subjective symptoms and outcome measures and a known risk of placebo effect. The RCTs were underpowered, inadequate with regard to duration of follow-up and performed on a problematically heterogeneous population. The trials did detect some positive effect of the treatment, but the effect was not statistically significant. This could be due to the fact that the treatment simply did not work, but the lack of statistical significance could also be due to the fact that it was an underpowered trial. (It is still plausible that the treatment actually worked, but the RCTs were too small to reveal this.) Ultimately, the Institute concluded that there was 'insufficient evidence': there was no proof that the washes 
were effective, nor was there any conclusive evidence that they were not. Actually, reimbursement decisions are frequently associated with uncertainty, as data on effectiveness, safety, etc. are seldom complete (Boon, Martins, \& Koopmanschap, 2015). Nonetheless, an occupational burden rests on the Institute to take a standpoint, despite this lack of certainty. In line with its general policy, the Institute concluded that without conclusive evidence of its effectiveness, the treatment cannot be reimbursed from public funds.

In court, patients and urologists challenged the appropriateness of the EBM method for making this decision. Before the judge, patients, urologists and policy makers negotiated whether either experimental knowledge from RCTs or experiential knowledge from the clinic ought to play a role in this reimbursement decision. How did a method, aimed at integrating experimental evidence, clinical expertise and patient experiences result instead in a lawsuit in which these forms of knowledge were weighed against one another? The lawsuit on bladder instillations highlights a paradox in EBM's success in both clinical practice and policy rooms. Originally, EBM aimed at an integrative assessment of the best external evidence with individual clinical expertise and patients' choice for decision making in the clinic (Sackett et al., 1996, p. 72). In the 1990s EBM also gained strong traction in policy-making circles. Making an integrative assessment for a national, stringent reimbursement decision in a policy room, however, means that such an assessment can no longer take place at individual case-level in the clinic. A negative reimbursement decision, unavoidably, means less discretionary room for professionals and patients, as a treatment is simply no longer (financially) available. This is how an evidence-based policy decision can, paradoxically, inhibit evidence-based decision making in clinical practice. In line with this, several authors have urged caution in the transportation of the EBM hierarchy from clinical medicine to the realm of policy making (Booth, 2010; Parkhurst \& Abeysinghe, 2014; Petticrew \& Roberts, 2003). Nonetheless, the court ruled that, with the EBM method, the Institute had given a correct application of the criterion of 'established medical science and medical practice'.

Studies have shown that the judiciary has long been reluctant to interfere in rationing decisions and has only recently evinced greater willingness to do so (Morales, 2015; Syrett, 2013; Wang, 2013). When they do 'courts are not normally empowered to substitute their view of the correct decision for that of the original decision maker, but only to ensure that the agency has the lawful authority to take the decision in question and that it complies with principles of procedural fairness' (Syrett, 2013, p. 7). In line with this, the Dutch court ruled 'procedural fairness' in case of the Institute's negative reimbursement decision. As an inevitable performative effect, the verdict also ratified the principles of EBM, the idea that 'objectivity' is obtained through experimental research, and that the value of an RCT exceeds that of other forms of knowledge.

The ICP and NVU challenged the evidential value of RCTs and of EBM's epistemic propriety, but to no avail. Their objections, however, resonate with the general critique on RCTs that a 'patient's unique and complex predicament is poorly served by applying a recommendation derived [...] from an average result in a select population sample' (Greenhalgh, 2002, p. 396). It seems that this holds especially true for RCTs performed on heterogeneous populations. IC is not a single 
condition. It is a term used in clinical practice to refer to a 'spectrum of urological symptoms' with different underlying causes (often unknown) (Offiah, McMahon, \& Oreilly, 2013, p. 1243). Those suffering from it are likely to respond differently to treatment (Nordling, Fall, \& Hanno, 2012; Offiah et al., 2013). For this reason, the ICP and NVU criticised the adequacy of RCTs as a means to gauge the effect of IC treatments. Acknowledging the issue, the Institute insisted throughout the lawsuit that if new subgroup studies were to become available, they could eventually reconsider their standpoint for specific groups of IC patients with proven benefit from this treatment. Yet, with the suspicions that rests on functional disorders and placebo-sensitive treatments, how viable is it that such studies will actually be taken up by the medical community? A scientific advisory member to the Institute remarked: "euhm, who would want to carry out an RCT involving something that doesn't work?" (notes\#30302014). The invitation to conduct multiple trials on sub-populations might be hypothetically rigorous, but it is often practically unrealistic. This type of practical consideration, however, was dismissed in court.

In court, the question of procedural fairness was largely conflated with the issue of doing 'epistemic' justice (Fricker, 2007). This term refers to justice in a way that all parties are duly recognised 'in their capacity as knower' and have a fair share of our 'collective interpretative resources' at hand in order to manifest what they know (Fricker, 2007, p. 1). Some scholars have warned that the principles of EBM are actually biased against patients (Greenhalgh et al., 2015) and that patients are easily and often 'epistemically marginalised' in the bureaucracy of health care systems (Carel \& Kidd, 2014, p. 530). For example, most clinical research has 'minimal patient input', which often entails that studies reflect the outcomes that matter to researchers, rather than those that matter to patients (Greenhalgh et al., 2015, p. 2; Wiering, de Boer, \& Delnoij, 2017). Furthermore, greatly reduced levels of credibility are assigned to patient testimonies (Carel \& Kidd, 2014) and EBM's hierarchy of evidence in particular tends to 'devalue patient experiences' (Greenhalgh et al., 2015 , p. 3). Those who are familiar with the clinical picture of functional disorders will recognise that this holds especially true for patients suffering from medically unexplained physical symptoms like (some cases of) IC, fibromyalgia, chronic fatigue syndrome, etc. This is also a group of patients with the least investigative resources at their disposal to make sense of their experiences (cf. Fricker, 2007). As mentioned earlier, IC and its treatment do not easily attract research attention or funds, in contrast to, for example, breast cancer. This means that IC patients are often left at the mercy of a few dedicated experts (see Shao et al., 2010). These are the 'epistemic margins' from which these patients have to argue their case: a highly reduced credibility and few entry points or resources to generate the required experimental evidence. This raises the question of what 'procedual fairness' signifies, if those who are subdued to these equal procedures have such unequal epistemic means at their disposal?

Lawsuits add to valuable learning processes (Syrett, 2013) and it seems that most of the learning is done outside the courtroom. Being at the Institute allowed us to witness the 'tinkering' done to improve EBM's principles for the practice of rationing decisions. As (Russell, Greenhalgh, Burnett, \& Montgomery, 2011, p. 2) noted, the rationing debate has long moved away from 'early 
naive faith' in the ability of EBM to straightforwardly guide us in complex rationing decisions. Since 2012, the Institute has been experimenting with conditional reimbursements, by nevertheless including treatments that do not fulfil the legal criterion of 'established medical science and medical practice' - for a specific period of time - into the insured package. This is subject to the condition that during that period data are collected on the efficacy of the care (Boon et al., 2015; College voor Zorgverzekeringen, 2012). Aware of the shortfalls of EBM's strict hierarchy, the Institute started implementing GRADE, a method that allows more flexibility within the original hierarchy and makes it possible to include patient panels in decision-making processes. Furthermore, the Institute now consults patient organisations more systematically in connection with rationing decisions. Together with the Institute for Medical Technology Assessment, the Institute drew up an 'appropriate evidence' questionnaire: a guide to whether RCTs can reasonably be expected in specific cases (Heymans, Kleijnen, \& Verstijnen, 2013). The above shows that, although judiciary interference is often experienced as a negative incident, lawsuits encourage institutional reflexivity.

The academic debate on health care rationing entails a long-established discussion about institutional processes, deliberative procedures and technical approaches (e.g. Daniels \& Sabin, 1997; Ham \& Roberts, 2003; Klein \& Maybin, 2012). We took an ethnographic approach that focussed on the working of these processes, procedures and rationality in practice, going back and forth between principles and policy ideals, and the 'messiness' of real-life decision making (cf. Gkeredakis et al., 2011; Jenkings \& Barber, 2004; Russell \& Greenhalgh, 2012, 2014). This type of analysis highlighted a set of practical issues with EBM that were not directly addressed in court. We argue that 'epistemic fairness' deserves our explicit attention, just as much as procedural soundness does, if not in court, then at least in academic and policy circles.

Acknowledgements The authors gratefully acknowledge all research participants at the National Health Care Institute for their valuable contributions to our study. The authors also thank two anonymous reviewers for their inspiring feedback on an earlier draft of this article. 


\section{REFERENCES}

Armstrong, D. (2002). Clinical autonomy, individual and collective: the problem of changing doctors' behaviour. Social science \& medicine (1982), 55(10), 1771-1777.

Bijker, W., Bal, R., \& Hendriks, R. (2009). The paradox of scientific authority: the role of scientific advice in democracies. Cambridge, Mass.: MIT Press.

Boon, W., Martins, L., \& Koopmanschap, M. (2015). Governance of conditional reimbursement practices in the Netherlands. Health Policy, 119(2), 180-185.

Booth, A. (2010). On hierarchies, malarkeys and anarchies of evidence. Health Information \& Libraries Journal, 27(1), 84-88.

Carel, H., \& Kidd, I. (2014). Epistemic injustice in healthcare: a philosophial analysis. Medicine, Health Care and Philosophy, 17(4), 529-540.

College voor Zorgverzekeringen. (2007). Beoordeling stand van de wetenschap en praktijk. Diemen: College voor Zorgverzekeringen (CVZ).

College voor Zorgverzekeringen. (2012). Rapport: Voorwaardelijke toelating/financiering van zorg. Diemen: College voor Zorgverzekeringen (CVZ).

College voor Zorgverzekeringen. (2013a). Achtergrondrapportage beoordeling stand van de wetenschap en praktijk. Blaasvloeistoffen met chondroitinesulfaat en/of hyaluronzuur (Vol. 2013084487). Diemen: College voor Zorgverzekeringen (CVZ).

College voor Zorgverzekeringen. (2013b). Update literatuursearch beoordeling stand van de wetenschap en praktijk blaasvloeistoffen met chondroitinesulfaat en/of hyaluronzuur (Vol. 2013142499). Diemen: College voor Zorgverzekeringen (CVZ).

Daniels, N., \& Sabin, J. (1997). Limits to Health Care: Fair Procedures, Democratic Deliberation, and the Legitimacy Problem for Insurers. Philosophy \& Public Affairs, 26(4), 303-350.

Daston, L., \& Galison, P. (1992). The image of objectivity: the talismanic image. Representations, 40(fall), 81-128. de Groot, G. (2006). GEZICHTSPUNT - De stand van de wetenschap en praktijk. Tijdschrift Voor Gezondheidsrecht, 30, 326.

de Laet, M. (2012). Anthropology as Social Epistemology? Social Epistemology, 26(3-4), 419-433.

Fricker, M. (2007). Epistemic injustice: power and the ethics of knowing. Oxford: Oxford University Press.

Gkeredakis, E., Swan, J., Nicolini, D., \& Scarbrough, H. (2011). Rational judgement revisited: Practices of deliberation in healthcare funding decisions. Paper presented at the OLKC conference, Hull University Business School.

Greenhalgh, T. (2002). Intuition and evidence - uneasy bedfellows? British Journal of General Practice, 52, 395-400.

Greenhalgh, T., Howick, J., \& Maskrey, N. (2014). Evidence based medicine: a movement in crisis? BMJ, 348, g3725.

Greenhalgh, T., Snow, R., Ryan, S., Rees, S., \& Salisbury, H. (2015). Six 'biases' against patients and carers in evidence-based medicine. BMC Medicine, 13, 200.

Guyatt, G., Oxman, A., Akl, E., Kunz, R., Vist, G., Brozek, J., . . J Jaeschke, R. (2011). GRADE guidelines: 1. Introduction - GRADE evidence profiles and summary of findings tables. Journal of Clinical Epidemiology, 64(4), 383-394. 
Ham, C., \& Roberts, G. (2003). Reasonable Rationing: International Experience of Priority Setting in Health Care. Maidenhead: Open University Press.

Heymans, J., Kleijnen, S., \& Verstijnen, I. (2013). Passend bewijs bij het bepalen van effectiviteit van interventies. NEDERLANDS TIJDSCHRIFT VOOR GENEESKUNDE, 157(17), 806-811.

Hoppe, R. (1999). Policy analysis, science and politics: from speaking truth to power to making sense together. Science and Public Policy, 26(3), 201-210.

Horstman, K. (2014). Struggling with science and democracy. Public health and citizenship in the Netherlands. In F. Huisman \& H. Oosterhuis (Eds.), Health and Citizenship. Political Cultures of Health in Modern Europe (pp. 191-208). London: Pickering and Chattoo Publication.

Jenkings, K., \& Barber, N. (2004). What constitutes evidence in hospital new drug decision making? Social science \& medicine, 58(9), 1757-1766.

Klein, R., \& Maybin, J. (2012). Thinking About Rationing. London: King's Fund.

Knorr Cetina, K. (1999). Epistemic Cultures: How the Sciences Make Knowledge. Cambridge, Mass.: Harvard University Press.

Knorr Cetina, K. (2007). Culture in global knowledge societies: knowledge cultures and epistemic cultures. Interdisciplinary Science Reviews, 32(4), 361-375.

Latour, B. (1993). We Have Never Been Modern. Cambridge, Mass.: Harvard University Press.

Leiber, S., Greß, S., \& Heinemann, S. (2015). Explaining Different Paths in Social Health Insurance CountriesHealth System Change and Cross-border Lesson-drawing between Germany, Austria and the Netherlands. Social Policy \& Administration, 49(1), 88-108.

Morales, L. (2015). Taking Facts Seriously: Judicial Intervention in Public Health Controversies. Public Health Ethics, 8(2), 185-195.

Moreira, T. (2011). Health care rationing in an age of uncertainty: a conceptual model. Social science \& medicine, 72(8), 1333-1341.

Moreira, T. (2012). The Transformation of Contemporary Health Care: the Market, the Laboratory, and the Forum. London: Routledge.

Nickel, J., Egerdie, B., Downey, J., Singh, R., Skehan, A., Carr, L., \& Irvine-Bird, K. (2009). A real-life multicentre clinical practice study to evaluate the efficacy and safety of intravesical chondroitin sulphate for the treatment of interstitial cystitis. BJU international, 103(1), 56-60.

Nickel, J., Egerdie, R., Steinhoff, G., Palmer, B., \& Hanno, P. (2010). A multicenter, randomized, double-blind, parallel group pilot evaluation of the efficacy and safety of intravesical sodium chondroitin sulfate versus vehicle control in patients with interstitial cystitis/painful bladder syndrome. Urology, 76(4), 804-809.

Nickel, J., Hanno, P., Kumar, K., \& Thomas, H. (2012). Second multicenter, randomized, double-blind, parallelgroup evaluation of effectiveness and safety of intravesical sodium chondroitin sulfate compared with inactive vehicle control in subjects with interstitial cystitis/bladder pain syndrome. Urology, 79(6), 1220-1225.

Nordling, J., Fall, M., \& Hanno, P. (2012). Global concepts of bladder pain syndrome (interstitial cystitis). World journal of urology, 30(4), 457-464.

Offiah, I., McMahon, S., \& Oreilly, B. (2013). Interstitial cystitis/bladder pain syndrome: diagnosis and management. International Urogynecology Journal: Including Pelvic Floor Dysfunction, 24(8), 1243-1256.

Parkhurst, J., \& Abeysinghe, S. (2014). What constitutes 'Good' evidence for public health and social policy making? From hierarchies to appropriateness. Social Epistemology Review and Reply Collective, 3(10), 34-46. 
Petticrew, M., \& Roberts, H. (2003). Evidence, hierarchies, and typologies: horses for courses. Journal of Epidemiology \& Community Health, 57(7), 527-529.

Riles, A. (2006). Documents: artifacts of modern knowledge. Ann Arbor: University of Michigan Press.

Russell, J., \& Greenhalgh, T. (2012). Affordability as a discursive accomplishment in a changing National Health Service. Social science \& medicine, 75(12), 2463-2471.

Russell, J., \& Greenhalgh, T. (2014). Being 'rational'and being 'human': how National Health Service rationing decisions are constructed as rational by resource allocation panels. Health, 18(5), 441-457.

Russell, J., Greenhalgh, T., Burnett, A., \& Montgomery, J. (2011). "No decisions about us without us"? Individual healthcare rationing in a fiscal ice age. BMJ, 342 (jun13 1), d3279.

Sackett, D., Rosenberg, W., Muir Gray, J., Haynes, R., \& Richardson, W. (1996). Evidence based medicine: what it is and what it isn't. $B M J, 312(7023), 71-72$.

Shao, Y., Shen, Z.-J., Rui, W.-B., \& Zhou, W.-L. (2010). Intravesical Instillation of Hyaluronic Acid Prolonged the Effect of Bladder Hydrodistention in Patients With Severe Interstitial Cystitis. Urology, 75(3), 547-550.

Syrett, K. (2013). Courts, Expertise and resource allocation: Is there a judicial 'legitimacy problem'? Public Health Ethics, 7(2), 112-122.

Van de Ven, W., \& Schut, F. (2008). Universal mandatory health insurance in the Netherlands: A model for the United States? Health Affairs, 27(3), 771-781.

Van de Ven, W., \& Schut, F. (2009). Managed competition in the Netherlands: Still work-in-progress. Health Economics, 18(3), 253-255.

Van Donk, R., \& Tusschenbroek, M. (2005). Kroniek rechtspraak zorgverzekeringsrecht. Tijdschrift Voor Gezondheidsrecht, 29, 563-572.

Wait, S., \& Nolte, E. (2006). Public involvement policies in health: exploring theirconceptual basis. Health Economics, Policy and Law, 1(2), 149-162.

Wang, D. (2013). Courts and health care rationing: the case of the Brazilian Federal Supreme Court. Health Economics, Policy and Law, 8(01), 75-93.

Weingart, P. (1999). Scientific expertise and political accountability: paradoxes of science in politics. Science and Public Policy, 26(3), 151-161.

Wiering, B. M., de Boer, D., \& Delnoij, D. (2017). Patient involvement in the development of patient-reported outcome measures: A scoping review. Health Expectations, 20(1), 11-23.

Zorginstituut Nederland. (2014). Factsheet. A well-balanced basic health care package. Health Care Coverage. Retrieved from: https://www.zorginstituutnederland.nl/binaries/content/documents/zinl-www/ documenten/rubrieken/english/1404-health-care-coverage-a-well-balanced-basic-health-care-package/ Health+Care+Coverage+\%28A+well-balanced+basic+health+care+package\%29.pdf 




\section{Strangers in the ER}

Based on: Moes, F., Houwaart, E., Delnoij, D., \& Horstman, K. (2018). "'Strangers in the ER": Quality indicators and third party interference in Dutch emergency care'. Journal of Evaluation in Clinical Practice,1-8. 


\section{ABSTRACT}

Rationale, aims and objectives This paper examines a remarkable dispute between Dutch insurers, hospitals, doctors and patients about a set of quality indicators. In 2013, private insurers planned to drastically reform Dutch emergency care using quality indicators they had formulated drawing from clinical guidelines, RCT's and systematic reviews. Insurers' plans caused much debate in the field of emergency care. As quality indicators have come to play a more central role in health care governance, the questions what constitutes good evidence for them, how they ought to be used and who controls them has become politically and morally charged. This paper is a case study of how a Dutch public knowledge institution, the National Health Care Institute, intervened in this dispute and how they addressed these questions.

Method We conducted ethnographic research into the knowledge work of the National Health Care Institute. Research entailed document analysis, participant observation, in-depth conversations and formal interviews with five key-informants.

Results The National Health Care Institute problematized not only the evidence supporting insurers' indicators, but also -and especially- the scope, purpose and use of the indicators. Our analysis shows the institute's struggle to reconcile the technical rationality of quality indicators with their social and political implications in practice. The institute deconstructed quality indicators as national standards and, instead, promoted the use of indicators in dialogue with stakeholders and their local and contextual knowledge.

Conclusions Even if quality indicators are based on scientific evidence, they are not axiomatically good or useful. Both proponents and critics of Evidence-based Medicine (EBM) always feared uncritical use of evidence by third parties. For non-medical parties who have no access to primary care processes, the type of standardized knowledge professed by EBM provides the easiest way to gain insights into 'what works' in clinical practice. This case study reminds us that using standardized knowledge for the management of health care quality requires the involvement of stakeholders for the development and implementation of indicators, and for the interpretation of their results. 


\section{INTRODUCTION}

In response to growing demands to achieve cost control, safety and transparency, quality indicators (or 'performance measures') have become increasingly important in the governance of health care. Quality indicators provide a means for care providers, decision makers and purchasers to measure, compare and improve the quality of care (Campbell, Roland, \& Buetow, 2000; Groene, Skau, \& Frølich, 2008; Hilarion et al., 2009). Experts agree, both in Dutch context (Beersen, Kallewaard, van Croonenborg, van Everdingen, \& van Barneveld, 2007; Koolman et al., 2012), and internationally (Kötter, Blozik, \& Scherer, 2012; Perera, Dowell, \& Crampton, 2012; Wollersheim et al., 2007), that indicators are ideally based on a clinical guideline, or -in absence of a guideline- on the best available scientific evidence with regard to quality of care. Although quality indicators are not directly linked to the clinical literacy movement that Evidence-based Medicine (EBM) originally set out to be (Djulbegovic \& Guyatt, 2017; Sackett, Rosenberg, Muir Gray, Haynes, \& Richardson, 1996), some have called indicators a 'branch' of EBM (Eddy, 2005) as they follow the same logic: clinical science can determine 'what works' and parameters based on these scientific findings can form an objective standard for provider behavior (Tanenbaum, 1995, 2012). Quality indicators developed according to an 'evidence-based approach' (Kötter et al., 2012; Perera et al., 2012) are generally reputed as a technical measuring device to evaluate the quality of care providers.

As quality indicators have come to play a more central role in health care governance, the questions what constitutes good evidence for these parameters, how indicators ought (and ought not) to be used and who controls them, has become politically and morally charged. In the Netherlands these questions were brought to a head when, in 2013, private insurers planned to drastically reform the sector using a set of quality indicators. The Association of Dutch Health Insurers had formulated these indicators drawing from clinical guidelines, RCTs and systematic reviews from the field of emergency care. Insurers used the indicators to negotiate which hospitals would preferably provide emergency services for multi-trauma, acute myocardial infarction, cerebrovascular accident, (ruptured) abdominal aortic aneurysm, natal care or hip fracture. The indicators substantiated insurers' argument that the centralization of complex emergency care in few specialized hospitals would lead to better and cheaper care.

Dutch doctors and professional organizations working in the field of emergency care strongly contested the accuracy and appropriateness of the indicators and insurers' use of them. "That [plan] is unacceptable and must disappear from the agenda... not because we have anything against health insurers, but because there is not a shred of evidence that this plan provides patients with better quality or accessibility", a spokesperson for hospitals in the Northern region claimed (Bart Kiers, 2014a). Others called insurers' plans "absolutely pretentious" (Joost Visser, 2014a). Hospitals and medical specialists were concerned about insurers' lack of medical-technical and practical insights into emergency care and feared for the quality of (and access to) emergency services (Bart Kiers, 2014b; Van Aartsen, 2014a). 
To solve the debate between insurers, hospitals, doctors and patients about the indicators, a public knowledge institution, the National Health Care Institute (Zorginstituut Nederland), intervened. This public knowledge institution is, amongst other things, responsible for the organization of understandable, comparable and unambiguous information about the quality of care. A special committee of medical specialists was installed at the institute to assess the quality indicators.

In this paper, drawing from ongoing ethnographic research into the work of the National Health Care Institute, we analyze the committee's assessment of said indicators and how they addressed the questions of what constitutes good evidence for quality indicators, how indicators ought to be used and who controls them. We conceptualize the work of the committee not as a technical exercise, but as socio-political work. In the next section we, first, explain our theoretical background and methodology. Then, we introduce the debate about the quality indicators in more detail and explain the role of the National Health Care Institute in the Dutch health care system. After that, we analyze how the committee assessed the quality indicators and problematized insurers' strategy to use quality indicators to centralize emergency care in the Netherlands.

\section{THEORY AND METHOD}

Drawing on insights from the field of Science and Technology Studies (STS), we conceptualize the controversy about quality indicators as a socio-political event. STS is an interdisciplinary field that examines the transformative power of science and technology to (re)arrange contemporary societies (Felt, Fouché, Miller, \& Smith-Doerr, 2017). Evidence is often invoked as a demarcation between objective knowledge and normative assumptions. Yet, quality indicators are developed at the very junction between fact and value. Therefore, in STS, standards like quality indicators are taken as socio-political tools and not as merely technical measuring devices (Timmermans \& Epstein, 2010). Indicators are socio-political entities, because they have financial, social, political and moral consequences and 'restructure the environments of which they become a part' (Timmermans \& Berg, 2003). For example, when health care managers use mortality rates of myocardial infarction as an indicator to measure the quality of hospitals, this can have far-reaching consequences for those working in those medical centers: it can redirect patient flows, reshuffle the social hierarchy between doctors, change funding opportunities and a hospital's legitimacy to work within the field of cardiology. As quality indicators 'define worth' and structure the world accordingly, they inevitably become 'a site of tensions, risk and uncertainty' (Lamont, 2012, p. 208). Taking this perspective, we study the assessment of quality indicators not as a technical exercise, but as socio-political knowledge work and a process of negotiating societal values.

While knowledge institutions are generally tasked to give technical advice, their work in the science-policy nexus inherently entails the balancing of scientific and public values (Bijker, Bal, \& Hendriks, 2009). This paper is based on ongoing ethnographic research into these socio- 
political knowledge work of the National Health Care Institute, a public knowledge institution in the Netherlands (Moes, Houwaart, Delnoij, \& Horstman, 2017). From October 2013 to September 2017, management of the institute provided the first author with an in-house desk (for 1-3 days a week), a digital workplace and access to archives to do intensive fieldwork within the institute. The first author attended public and closed meetings of internal working groups (weekly), the executive board (two-weekly), as well as expert meetings of the institute's advising committees (Package Advisory Committee, Quality Council, Scientific Advisory Committee, Health Care Professions Committee), staff fora and informal lunches. As the controversy about quality indicators for emergency care was a flagship case for the National Health Care Institute, we decided to focus part of the study on this case.

In three consecutive stages, the first author collected empirical data between October 2013 and August 2016 on the debate about insurers' quality indicators and the institute's engagement with it through in-depth conversations, document analysis, participant observation and formal interviews. First of all, to get familiar with the case, the fist author had informal conversations with involved staff members and directors at the institute. She delved into the institute's archives to retrieve all relevant written material referring to the case, including internal and external emails, minutes of meetings, official and internal documents. The author also searched for public reports and articles in newspapers and professional journals that reported about insurers' indicators for emergency care and the institute's work in this regard. This resulted in a dossier of over 450 pages. Secondly, to gain more direct experience with the institute's involvement in the debate, the first author attended an executive board meeting on the issue, plus four meetings of the Quality Council in which insurers' indicators were discussed. She also attended two days of consensus meetings that the institute organized with field parties to discuss quality standards for emergency care. Thirdly, in order to triangulate our observations and documentary research, we did formal, semistructured interviews. Through 'purposive' sampling (Green \& Thorogood, 2004) of information-rich informants we selected five key persons: two members of the Quality Council, two staff members and the council's scientific secretary.

The first, second and fourth author engaged in an iterative process of joint close reading of field notes, reports, policy documents, minutes and interview transcripts. (The third author engaged in discussions of the final drafts.) The leading questions in the analysis were how the institute engaged with the debate about quality indicators, what the committee of medical specialists decided about the accuracy and appropriateness of these indicators for the (re)organization of Dutch emergency services and why they did so. We aimed to understand how, in the Netherlands, a public knowledge institution addressed the simultaneously technical and socio-political questions of what constitutes good evidence for quality indicators, how indicators ought to be used and who controls them. Ultimately, as a 'member-check', we sent a written version of the analysis to involved staff members and interviewees to test our analysis with them (face-to-face/email/ telephone). 


\section{RESULTS}

\section{The ER Controversy in the Netherlands}

Private insurance companies play a prominent role in the Dutch health care system of managed competition, since the introduction of the 2006 Health Insurance Act (Zorgverzekeringswet). A system of universal mandatory health insurance obliges all citizens to take out basic health insurance provided by private insurers. Competition was introduced on two levels. Citizens have to choose between competing health insurance companies during a yearly open enrollment period and insurers are expected to negotiate price, service and quality of care with providers on behalf of their insured clients (Van de Ven \& Schut, 2008, p. 779). Despite heavy regulation, insurers and hospitals are free to negotiate prices and selectively contract a range of hospital care products. 'Selective contracting' is a vital aspect of the Health Insurance Act. Insurers can steer their customers away from hospitals that do not reduce their prices or improve quality. This way, selective contracting is thought to stimulate both quality and efficiency (Bijlsma, Boone, \& Zwart, 2009).

It was in their role as selective contractors that in 2013, the Association of Dutch Health Insurers (Zorgverzekeraars Nederland, hereafter 'ZN') published its 'Quality Vision for Emergency Care' (Zorgverzekeraars Nederland, 2013), deriving and formulating quality indicators from the scientific literature, to reorganize emergency services along efficiency lines. According to ZN, the centralization of complex emergency care in few specialized hospitals would lead to better and cheaper care. In its report, ZN focused on urgent neurological, cardiologic and vascular surgical care, traumatology and obstetrics. (Emergency pediatrics and emergency psychiatry were not addressed in ZN's plans, because pediatric care in the Netherlands is already centralized, and psychiatric care has its own specific organizational structure (Zorgverzekeraars Nederland, 2013)).

The report contained six specific sets of quality indicators: for measuring the quality of care for multi-trauma and hip fracture (traumatology), acute myocardial infarction (emergency cardiology), cerebrovascular accident (emergency neurology), (ruptured) abdominal aortic aneurysm (vascular surgical care), and natal care (obstetrics). For example, for multi-trauma the insurers specified that good care required: a trauma center with a treatment team, 24-hour accessibility of an internal trauma team; the presence in hospital of the required specialists within 15 minutes; mobile medical team (MMT); adequate facilities (like a CT-scan in the emergency room; an operating theatre and intensive care unit next to emergency room). Quality of care would be measured based on 30-days mortality after (multi-)trauma. In order to guarantee quality, a hospital was required to treat 240-480 multi-trauma patients per center, per year (Zorgverzekeraars Nederland, 2013, p. 17).

ZN substantiated the indicators by referring to Dutch, European and international guidelines and a vast number of scientific publications, including RCT's, systematic reviews and cohort studies. According to ZN, private insurers could use these indicators for selective contracting of emergency cardiology, emergency neurology, traumatology, urgent vascular surgical care and obstetrics. They also proposed to centralize these services in specialized centers if the proposed quality indicators indicated that this was desirable (Zorgverzekeraars Nederland, 2013, p. 5). By the end of 2013, 
insurers started negotiating (using said indicators), which hospitals would maintain a fully equipped emergency unit, and which hospitals would lose part of their emergency care.

A journalist reported: 'In Rotterdam most complex emergency care will move to Erasmus Medical Center. Soon four times as many people will be going there with a stroke. Five hospitals will lose stroke care' (Van Aartsen, 2014b)

A hospital director stated: "Emergency room closed, no more obstetrics, nor stroke care, no balloon angioplasty, and even broken hip operations will have to be done elsewhere" (Joost Visser, 2014a)

The plans caused much debate in the field of emergency care. From the exchange of arguments in professional media, it became clear that the accuracy and use of ZN's indicators was contested by Dutch professional organizations. The Dutch Hospital Association (NVZ) emphasized that such indicators need to be well-founded and was concerned about insurers' lack of medical-technical and practical insights into emergency care (Bart Kiers, 2014b; Van Aartsen, 2014a). NVZ criticized that insurers gave little credence to the fact that it is often difficult to predict a patient's diagnosis before he/she enters a hospital. A patient with acute abdominal pain could suffer from a wide variety of conditions ranging from gastroenteritis to abdominal aortic aneurysm. The director of the NVZ rhetorically stated: "will concentration mean, for instance, in respect of abdominal aneurysms, that all patients with stomach pain must be sent immediately to hospitals with an emergency room for complex acute care?" (Joost Visser, 2014a). In another line of critique, the Dutch Order of Medical Specialists (OMS) claimed that a hospital that loses its contract for the treatment of, for example, myocardial infarction would have to deal with a 'cascading effect', meaning that the general cardiology in that hospital could deteriorate or vanish completely from that hospital too. The OMS urged that "a hospital is a careful construction of building bricks, removing just one brick can cause everything to collapse" (Joost Visser, 2014b). Of course, apart from quality concerns, hospitals and specialists had their own interests at stake in the debate.

In 2014, Dutch professional organizations, the NVZ and OMS, appealed to the Authority for Consumers and Markets (ACM) to express their concerns about insurers' plans. ACM is the regulatory institute that oversees markets, fair competition and consumer rights. The regulatory body was concerned that insurers' plans to concentrate emergency care could 'reduce the choices open to patients and insured clients' and warned insurers that 'implementing the proposed plans could contravene the Competition Law' (Autoriteit Consument en Markt, 2014). ACM decided that curtailment of consumer choice was only acceptable if it would lead to a considerable quality gain for patients. The regulatory body ordered insurers to demonstrate that this was indeed the case by providing 'independent and well-founded quality standards for emergency care' (Autoriteit Consument en Markt, 2014) or to organize broad support for the indicators in the field. As ZN 
required quality indicators for their practice of selective contracting, it turned to the National Health Care Institute to organize broadly supported quality indicators.

\section{The role of the National Health Care Institute}

The National Health Care Institute is, amongst other things (such as the management of the Dutch basic benefits package (Moes et al., 2017)), lawfully tasked to organize understandable, comparable and trustworthy information about the quality of care. Since the introduction of the 2006 Health Insurance Act, reliable quality information has become a crucial ingredient for the proper functioning of the Dutch system of managed competition (Enthoven \& Van de Ven, 2007). Quality information provides both consumers and insurers with comparable information about the performance of health care providers in order to negotiate price and quality (Delnoij, Rademakers, \& Groenewegen, 2010). In the years that followed the introduction of the Health Insurance Act, the health care sector developed all kinds of quality information. The multiplicity of indicators, however, actually blocked oversight and hindered informed consumer choice (Algemene Rekenkamer, 2013, p. 6). In response to this problem, the Minister of Health proposed to centralize the organization of quality information and on April $1^{\text {st }} 2014$ the National Health Care Institute was established. The institute was lawfully tasked to organize understandable, comparable and trustworthy information about the quality of care, and has what is called 'overriding authority': it can authoritatively lay down indicators when field parties fail to deliver them or to reach consensus. If the institute uses this 'overriding authority', the development of quality standards or indicators is put in the hands of the Quality Council, an advisory committee of the National Health Care Institute.

It was from their role as coordinator of quality information that the institute was asked to assess the quality indicators for emergency care. After several rounds of meetings and consultations facilitated by the institute, the professional organizations of insurers (ZN), patients (NPCF), medical specialists (OMS), hospitals (NVZ) and academic hospitals (NFU) failed to reach agreement on insurers' quality indicators for emergency care. That is to say, the specialists, (academic) hospitals and patient organizations submitted their own revised set of indicators, but without the support of the insurers. As a result, the institute used its 'overriding authority' and asked the Quality Council to review the revised indicator set. The council, in turn, installed a group of thirteen medical specialists to do the formal assessment. To safeguard representativeness, the council selected specialists from different relevant medical fields in emergency care and from both peripheral and academic hospitals. Also, the selected specialists could not be formally affiliated to any of the stakeholders that proposed the indicators. Next, we show how the committee assessed the quality indicators and problematized insurer's strategy to use them to centralize emergency services in the Netherlands.

\section{Problem 1 | Scope of indicators}

The committee installed by the Quality Council was tasked to assess whether the indicators were 'evidence-based' and adequate for measuring the quality of emergency care. From our interviews, observations and documentary research it became clear that the committee actually found that 
most of the indicators were, as staff commented, "not unreasonable", as there was sufficient evidence supporting the indicators. The accuracy of the individual indicators was not the primary problem according to the committee. They did have a problem with the scope of the indicators. Next we illustrate this with an example.

Insurers proposed to measure, for example, the quality of care for acute myocardial infarction (AMI) by looking at how a hospital scored at the following:

a) availability of a Cardiovascular Intervention Center, Emergency Cardiovascular Care (ECC), Cardiac Care Unit (CCU), and a cardiac rehabilitation ward (structural indicators);

b) percentage of ST-Elevation Myocardial Infarction (STEMI) treated with primary percutaneous coronary intervention ( $\mathrm{PCl}$ ), and hospital's percentage of medication after hospital discharge for AMI (process indicators);

c) AMI mortality rate after 30 days, patient-reported functional health after AMI, and percentage of $\mathrm{PCl}$ re-interventions (outcome indicators);

d) hospital's / cardiologist's annual number of PCl-treatments performed (600 PCl's per hospital /150 PCl's per cardiologist) (volume norms) (Zorgverzekeraars Nederland, 2013, pp. 23-25).

Our interviewees explained that, while these indicators make sense in themselves, the quality of the treatment of myocardial infarction depends on the entire trajectory from incident to aftercare, not just on what happens inside a hospital. Staff members said that insurers' indicators "started in the hospital", while "an emergency pathway starts with a demand for emergency care, so it starts at home perhaps, or in the street... or under a car .... A committee member explained in an interview that the quality of treatment for myocardial infarction,

"starts with a patient raising the alarm in good time, after which the GP takes a look, who then refers the patient to hospital in good time, and all of that finally determines the outcome of a myocardial infarction. If you judge a hospital based on, for instance, survival rates for myocardial infarction, well then you would also have to take into account the part of the chain before the hospital... if people call in the GP too late, or the GP doesn't do his job properly, you will be running the risk that indicators will work against hospitals. Well, you have to realize this, if you are going to make use of this sort of indicator"

The committee therefore broadened the scope of the indicators. In their final report 'Excellency Demanded for Emergency Care' (Zorginstituut Nederland, 2015), the committee systematically supplemented indicators focusing on hospital performance with indicators regarding the organizational chain of emergency services (including, for example, regional ambulance services, triage systems and urgent transferal). For AMI, for example, the committee included 'written 
agreements about cooperation with partners in the organizational chain' of care for AMI (Zorginstituut Nederland, 2015, p. 20). By doing so, the committee emphasized the importance of the network in which an emergency patient makes a journey. It is within this network that diagnoses are made and the quality of treatments takes shape. By adding 'chain agreements' to the set of quality indicators, the committee required that the quality of hospitals was not only measured by hospitals' 'in-door' performance, but also measured by looking at hospitals' abilities to organize and maintain a wellfunctioning network in the region.

\section{Problem 2 | Purpose of indicators}

As we stated before, the committee found that most quality indicators were supported by evidence. This was, however, not the case for the so-called 'optimum' volume norms proposed by insurers. The committee problematized not only the lack of evidence for insurers' volume norms, but also the purpose behind the norms. Next we elaborate on the committee's problems with insurers' 'optimum' volume norms, and why they proposed 'minimum' volume norms instead.

The idea behind volume norms is that hospitals treating a bigger volume of patients get better treatment results, thus, leading to better quality and efficiency. ZN wrote in their report that

'large volumes will make it possible to use an expensive infrastructure [...] more effectively. At the same time, a larger volume will also deploy personnel (specialists and specialist nurses) that is scarce (and becoming even scarcer) more effectively' (Zorgverzekeraars Nederland, 2013, p. 7).

ZN proposed 'optimum' volume norms describing at what number of patients the quality and efficiency of emergency services would be optimized. ZN recommended for stroke, for example, that a hospitals would have to treat at least 350 patients with cerebrovascular accident (CVA) per year (Zorgverzekeraars Nederland, 2013, p. 21). For (ruptured) abdominal aortic aneurysm (rAAA), insurers claimed that hospitals would have to treat at least 15 ruptured abdominal aortic aneurysms and a total of 33 abdominal aortic aneurysms or more to deliver good quality care for these patients (Zorgverzekeraars Nederland, 2013, p. 27). According to ZN, their 'optimum' volume norms were 'an estimate of what is a sensible level of emergency care concentration in order to book both quality and efficiency gains' (Zorgverzekeraars Nederland, 2013, p. 7).

Insurers' volume norms, however, were strongly contested by field parties. According to staff members the "biggest stumbling blocks really were the volume criteria" in the debate about quality indicators. The committee's secretary recalled that "the volume norms... that was what led to discussions", because these norms had far-reaching consequences. The chairperson to the committee explained in an interview that if insurers would have selectively contracted emergency care on the basis of these optimum volume norms, this "would have resulted in a lot of casualty departments shutting down ... it would have been a ravage ... lots of them would have closed because they were simply unable to fulfil the criteria". 
The committee installed by the Quality Council was tasked to assess whether the volume norms were 'evidence-based' and adequate for measuring the quality of emergency care. According to the committee's secretary there was "insufficient evidence" supporting these optimum norms. It became clear from our interviews, observational and documentary research that the committee did not only have a problem with the lack of evidence for insurers' volume norms, but also with the purpose behind these 'optimum' norms. A committee member explained that "based on a principle of solidarity or collectivity, you might actually think that [care] should be 'good enough' everywhere" and not necessarily 'optimal'.

In their final report, the committee proposed 'minimum' volume norms following the advice of the scientific associations of the different fields of medical expertise. Staff members explained that scientific associations "often stipulate minimum norms" and that "these are often so low that everyone fulfils them". The purpose of minimum norms is not to optimize quality and efficiency, but to secure a minimum level of safe and reliable emergency services. Working from this idea, the committee lowered insurers' optimal volume norms to minimum norms. For CVA, for example, the committee followed the Dutch Association for Neurology (NVN) that advised a minimum volume of 100 patients with cerebrovascular accident (CVA) per year per hospital. At the same time, the committee raised the minimum volume norm for (ruptured) abdominal aortic aneurysm (RAAA). Working from that same idea that volume norms serve the purpose of securing safe and reliable emergency services, the chairperson explained, the committee thought: "with aneurysm for example... this is a life threatening procedure... and if you do less than 20 of those in a hospital [per year].... that is just unwise." By stipulating minimum volume norms, the chairperson to the committee claimed that

on the one hand: we "made sure no damage was inflicted by units having to close down or being no longer able to provide care, units that I felt - we all felt - were capable of providing good care..."

on the other hand: "our norms prevented aneurysm operations from still being carried out in [city X], while they really should be taken to [city $Y$ ], because then the survival of these people is actually much higher"

Apart from the lack of evidence supporting the optimum volume norms of $Z N$, the committee problematized the purpose behind optimum norms. Instead they stipulated minimum norms, following the scientific associations of the respective field of medical expertise. While the purpose of insurers' optimum volume norms was to organize optimal quality and efficiency in emergency care and thus optimize welfare economics, the purpose of the minimum volume norms endorsed by the committee echoed the rationale of another public responsibility: a collective duty to secure access and availability of 'good-enough' emergency services for all. 


\section{Problem 3 | Use of indicators}

Finally, our research showed that the committee problematized insurers' unilateral use of the quality indicators. In their final report, the committee revised and laid down quality indicators for emergency care and stressed the importance of collectively weighing the applicability of the indicators and their fit with regional context (Zorginstituut Nederland, 2015, p. 15).

ZN designed quality indicators to enable 'insurers to shape their selective care purchasing' (Zorgverzekeraars Nederland, 2013, p. 5). On the basis of these indicators, insurers had started to compare hospitals in the different regions and started negotiating selective contracts. Field parties, however, considered insurers' initiative a top-down exercise. A hospital director recalled the negotiations with insurers: "there were lists with green and red ticks" and objected: "you can't throw down some sort of blueprint from your ivory tower"(Bart Kiers, 2014a).

According to the chairperson to the committee, the problem was that insurers were acting unilaterally using standardized knowledge, while they were actually at a distance from the primary care process and had no hands-on experience of emergency care:

"This was just one party... and a party that was at a considerable distance... and because [the insurers] are at such distance, their only weapon is population data from the evidence-based medicine world... which is standardized... if someone has no hands-on experience and has never actually been to an emergency room... then these are the only available data for him to use. But, then he forgets the knowledge sources that are somewhat harder to exploit for him, but that are very important too..."

With "evidence-based medicine as the foundation of the quality movement", the chairperson claimed, focus has moved to "standardized protocol quality". But in applying such standards "local data are important" and collaboration in the region is necessary to exploit sources of local, tacit and contextual knowledge. In their final report, the committee stressed that the quality indicators they proposed were to be used by professionals to discuss collectively how best to organize emergency services in the particular regions (Zorginstituut Nederland, 2015, p. 15). Rather than a unilateral tool for selective contracting, the committee proposed the indicators as a tool for dialogue and learning, allowing regional parties to gain insight into opportunities to improve situational quality.

\section{DISCUSSION}

The National Health Care Institute is a public knowledge institute that is, amongst other things, lawfully tasked to organize trustworthy information about the quality of care. We studied how this body intervened in a debate about the quality of emergency care and in that context we analyzed their assessment of quality indicators as socio-political knowledge work. Our analysis of their work showed a struggle to reconcile the technical rationality of quality indicators with their social and 
political implications in practice. The committee installed by the National Health Care Institute problematized not only the evidence behind some of the quality indicators, but also and especially their scope, purpose and use. According to the chairperson, the committee "put the evidence into a bit of perspective".

In scientific literature on the development of quality indicators it has been recognized (albeit in different wording) that indicators operate at the very junction between fact and value (de Koning, Kallewaard, \& Klazinga, 2007; Hilarion et al., 2009; Kötter et al., 2012; Perera et al., 2012). Although this paper describes a single case, it serves as a real-life example of what experts on indicator development have long recognized: that even if quality indicators are based on solid scientific evidence, they are not 'axiomatically good' (Freeman, 2002) or useful. Indicators developed for one purpose (e.g. measuring the quality of care for myocardial infarction or stroke) may be inappropriate for a different application (e.g. measuring the ideal level of concentration of these emergency services) (Perera et al., 2012). Furthermore, indicators can serve a wide array of values; not all of them equally desirable from the perspective of public health services. The proper use of quality indicators in a public context requires the involvement of stakeholders in the development of indicators, the collective formulation of objectives, and the use of local, tacit and contextual knowledge to aid both the implementation of indicators and the interpretation of their outcome (Freeman, 2002).

The socio-political work of the National Health Care Institute reflects specific characteristics of Dutch health care. The Netherlands has a system of managed competition in which insurers are expected to stimulate quality and efficiency in health care through, for example, selective contracting. Private insurers, however, still lack public trust in the Netherlands (Bes, Wendel, Curfs, Groenewegen, \& de Jong, 2013). The Dutch healthcare system is highly regulated and private insurers operate under strong legislation and are critically watched by all kinds of professional organizations and state institutions. The Dutch have, furthermore, a culture of consensual policymaking called 'polderen' (Helderman, De Kruijf, Verheij, \& Van Thiel, 2014, p. 1). Not every health care system is as much disposed to inclusive dialogue. The attempt by insurers to corroborate concentration plans with quality indicators reminds us that -with the marketization of healthcarequality indicators can easily align to match the logic of the market. This case study shows that it requires specific knowledge work and dialogue to realign such scientific tools to make them socially robust and serve a public purpose.

\section{CONCLUDING REMARKS}

Quality indicators are not directly linked to Evidence-based Medicine. They do, however, follow the same logic: clinical science - ideally statistical population research- can determine 'what works' and parameters based on these scientific findings can form an objective standard for provider behavior. Both proponents (Djulbegovic \& Guyatt, 2017; Guyatt, Meade, Jaeschke, Cook, \& Haynes, 2000; 
Sackett et al., 1996) and critics (Charlton, 1997; Hunter, 1996) of EBM have long problematized the uncritical use of statistical population research and the evaluation of medical practices based on probabilistic knowledge (Tanenbaum, 1995, 2012). However, for non-medical parties who have no access to the primary care processes, this type of knowledge provides the easiest way to gain insights into 'what works' in clinical practice. EBM's emphasis on standardized, impersonal research procedures suggests the possibility of separating 'expertise from expert and knowledge from knower' and that it is possible to distill 'medical truth outside the clinical encounter' (Tanenbaum, 1995 , p. 102). As a result of this uncoupling of expert and expertise, the use of statistical population research has given outsiders to health care the scientific authority to act independently in a field where - originally - they had none (Porter, 1995). While outside interference into clinical practice is of all times (Rothman, 1991), critics (Charlton, 1997, p. 87; Hunter, 1996, p. 6) feared that EBM's standardization of medical knowledge, would allow 'strangers' -like inspectorates, policymakers or insurers - to 'regulate the field of healthcare and hold it accountable using evidence-based parameters formulated by the professions' themselves (Timmermans \& Berg, 2003, p. 20). This case study reminds us that the interplay of forces between the uncritical use of standardized medical knowledge, the growing use of quality indicators, and the marketization of health care continues to require our explicit attention.

Acknowledgements The authors gratefully acknowledge all research participants at the National Health Care Institute for their valuable contributions to our study. The authors also thank two anonymous reviewers for their feedback on an earlier draft of this article. 


\section{REFERENCES}

Algemene Rekenkamer. (2013). Indicatoren voor kwaliteit in de zorg. 's-Gravenhage: Sdu Uitgevers.

Autoriteit Consument en Markt. (2014). ACM wijst zorgverzekeraars op mededingingsrisico bij uitvoering gezamenlijke plannen spoedeisende zorg [Press release]. Retrieved from https://www.acm.nl/nl/publicaties/ publicatie/13132/ACM-wijst-zorgverzekeraars-op-mededingingsrisico-bij-uitvoering-gezamenlijke-plannenspoedeisende-zorg/

Beersen, N., Kallewaard, M., van Croonenborg, J., van Everdingen, J., \& van Barneveld, T. (2007). Handleiding indicatorontwikkeling: ZonMw.

Bes, R., Wendel, S., Curfs, E., Groenewegen, P., \& de Jong, J. (2013). Acceptance of selective contracting: the role of trust in the health insurer. BMC Health Services Research, 13(1), 375.

Bijker, W., Bal, R., \& Hendriks, R. (2009). The paradox of scientific authority: the role of scientific advice in democracies. Cambridge, Mass.: MIT Press.

Bijlsma, M., Boone, J., \& Zwart, G. (2009). Selective Contracting and Foreclosure in Health Care Markets. CentER Discussion Paper Series No. 2009-89, TILEC Discussion Paper No. 2009-045.

Campbell, S., Roland, M., \& Buetow, S. (2000). Defining quality of care. Social science \& medicine, 51(11), 1611-1625.

Charlton, B. G. (1997). Restoring the balance: evidence-based medicine put in its place. Journal of Evaluation in Clinical Practice, 3(2), 87-98.

de Koning, J., Kallewaard, M., \& Klazinga, N. (2007). Prestatie-indicatoren langs de meetlat - het AIRE instrument. TSG, 85(5), 261-264.

Delnoij, D., Rademakers, J., \& Groenewegen, P. (2010). The Dutch Consumer Quality Index: an example of stakeholder involvement in indicator development. BMC Health Services Research, 10(88).

Djulbegovic, B., \& Guyatt, G. (2017). Progress in evidence-based medicine: a quarter century on. The Lancet, 390(10092), 415-423.

Eddy, D. (2005). Evidence-Based Medicine: A Unified Approach. Health Affairs, 24, 9.

Enthoven, A., \& Van de Ven, W. (2007). Going Dutch - Managed-competition health insurance in the Netherlands. The New England Journal of Medicine(357), 2421-2423.

Felt, U., Fouché, R., Miller, C., \& Smith-Doerr, L. (2017). The handbook of science and techno-logy studies. Cambridge; Mass: MIT Press.

Freeman, T. (2002). Using performance indicators to improve health care quality in the public sector: a review of the literature. Health Services Management Research, 15(2), 126-137.

Green, J., \& Thorogood, N. (2004). Qualitative Methods for Health Research. London: SAGE Publication.

Groene, O., Skau, J., \& Frølich, A. (2008). An international review of projects on hospital performance assessment. International Journal for Quality in Health Care, 20(3), 162-171.

Guyatt, G., Meade, M., Jaeschke, R., Cook, D., \& Haynes, R. (2000). Practitioners of evidence based care: Practitioners of evidence based care. Not all clinicians need to appraise evidence from scratch but all need some skills. BMJ, 320(7240), 954-955.

Helderman, J., De Kruijf, J., Verheij, J., \& Van Thiel, S. (2014). Dike-Reeve of the health care polder. A politicalsociological analysis of the realisation of the National Health Care Institute against a backdrop of a changing policy agenda and changing political-administrative and societal relations. Diemen: Zorginstituut Nederland. 
Hilarion, P., Suñol, R., Groene, O., Vallejo, P., Herrera, E., \& Saura, R. (2009). Making performance indicators work: The experience of using consensus indicators for external assessment of health and social services at regional level in Spain. Health Policy, 90(1), 94-103.

Hunter, D. (1996). Rationing and evidence-based medicine. Journal of Evaluation in Clinical Practice, 2(1), 5-8.

Kiers, B. (2014a, 13 March 2014). Ziekenhuizen maken gehakt van spoedplan. Zorgvisie

Kiers, B. (2014b, 16 July 2014). ACM haalt streep door concentratie spoedzorg. Zorgvisie

Koolman, X., Zuidgeest, M., Visser, J., Appelman, M., Beersen, N., \& Berg, M. (2012). Indicatorstandaard. Methodologische criteria voor de ontwikkeling van betrouwbare kwaliteitsindicatoren in de zorg. Retrieved from https://www.zorginzicht.nl/kennisbank/Publishinglmages/Indicatorstandaard\%202.0.pdf

Kötter, T., Blozik, E., \& Scherer, M. (2012). Methods for the guideline-based development of quality indicators - a systematic review. Implementation Science, 7(1), 21.

Lamont, M. (2012). Toward a Comparative Sociology of Valuation and Evaluation. Annual Review of Sociology, 38, 201-221.

Moes, F., Houwaart, E., Delnoij, D., \& Horstman, K. (2017). Contested evidence: a Dutch reimbursement decision taken to court Health economics, policy, and law, 12(3), 325-344.

Perera, R., Dowell, A., \& Crampton, P. (2012). Painting by numbers: A guide for systematically developing indicators of performance at any level of health care. Health Policy, 108(1), 49-59.

Porter, T. (1995). Trust in Numbers: the Pursuit of Objectivity in Science and Public Life. Princeton: Princeton University Press.

Rothman, D. (1991). Strangers at the bedside: A history of how law and bioethics transformed medical decision making. New York, NY: BasicBooks.

Sackett, D., Rosenberg, W., Muir Gray, J., Haynes, R., \& Richardson, W. (1996). Evidence based medicine: what it is and what it isn't. BMJ, 312(7023), 71-72.

Tanenbaum, S. (1995). Getting there from here: evidentiary quandaries of the US outcomes movement. Journal of Evaluation in Clinical Practice, 1(2), 97-103.

Tanenbaum, S. (2012). Improving the quality of medical care: the normativity of evidence-based performance standards. Theoretical medicine and bioethics, 33(4), 263-277.

Timmermans, S., \& Berg, M. (2003). The Gold Standard: the challenge of evidence-based medicine and standardization in health care. Philadelphia, PA.: Temple University Press.

Timmermans, S., \& Epstein, S. (2010). A World of Standards but not a Standard World: Toward a Sociology of Standards and Standardization. Annual Review of Sociology, 36, 69-89.

Van Aartsen, C. (2014a, 5 February 2014). NVZ clasht met zorgverzekeraars over spoedzorg. Zorgvisie.

Van Aartsen, C. (2014b, 31 January 2014). Vijf Rotterdamse ziekenhuizen verliezen beroerte-zorg. Zorgvisie.

Van de Ven, W., \& Schut, F. (2008). Universal mandatory health insurance in the Netherlands: A model for the United States? Health Affairs, 27(3), 771-781.

Visser, J. (2014a, 12 February 2014). Concentratie spoedzorg ligt zwaar op de maag. Medisch Contact.

Visser, J. (2014b, 30 July 2014). Concentratie van spoedzorg is hooguit afgeremd. Medisch Contact.

Wollersheim, H., Hermens, R., Hulscher, M., Braspenning, J., Ouwens, M., Schouten, J., . . Grol, R. (2007).

Clinical indicators: development and applications. The Netherlands Journal of Medicine, 65(1), 15-22. 
Zorginstituut Nederland. (2015). Spoed Moet Goed: Indicatoren en Normen voor Zes Spoed-indicaties. Diemen: Zorginstituut Nederland.

Zorgverzekeraars Nederland. (2013). Kwaliteits-visie Spoedeisende Zorg. Hoofdrapport. Zeist: Zorgverzekeraars Nederland. 

Chapter 3

\section{Collective Constructions of 'Waste'}

Based on: Moes, F., Houwaart, E., Delnoij, D., \& Horstman, K. 'Collective Constructions of 'Waste': Epistemic practices for disinvestment in the context of Dutch Social Health Insurance'.

[Revisions submitted] 


\section{ABSTRACT}

Background Faced with growing budget pressure, policymakers worldwide recognize the necessity of strategic disinvestment from ineffective, inefficient or harmful medical practices. However, disinvestment programs face substantial social, political and cultural challenges: mistrust, struggles for clinical autonomy or stakeholders' reluctance to engage in what can be perceived as 'rationing' Academic literature says little about effective strategies to address these challenges. This paper provides insights on this matter. We analyzed the epistemic work of a group of policymakers at the National Health Care Institute on what was initially a disinvestment initiative within the context of the Dutch basic benefits package: the 'Appropriate Care' program. The Institute developed a strategy using national administrative data to identify and tackle low-value care covered from public funds as well as potential underuse, and achieve savings through improved organization of efficiency and quality in health care delivery. How did the Institute deal with the socio-political sensitivities associated with disinvestment by means of their epistemic work?

Method We conducted ethnographic research into the National Health Care Institute's epistemic practices. Research entailed document analysis, non-participant observation, in-depth conversations, and interviews with key-informants.

Results The Institute dealt with the socio-political sensitivities associated with disinvestment by democratizing the epistemic practices to identify low-value care, by warranting data analysis by clinical experts, by creating an epistemic safe space for health care professionals who were the object of research into low-value care, and by de-emphasizing the economization measure. Ultimately, this epistemic work facilitated a collaborative construction of problems relating to lowvalue care practices and their solutions.

Conclusions This case shows that - apart from the right data and adequate expertise disinvestment requires clinical leadership and political will on the part of stakeholders. Our analysis of the Institute's Appropriate Care program shows how the epistemic effort to identify low-value care as well as potential underuse became a co-construction between policymakers, care providers, patients and insurers of problems of 'waste' in Dutch social health insurance. This collective epistemic work gave cognitive, moral and political standing to the idea of 'waste' in public health expenditure. 


\section{BACKGROUND}

The desire to maintain more sustainable health care systems is placing growing pressure on budget-holders, worldwide, to reassess currently funded medical practices and potentially release resources to higher priority areas (Mitton, Dionne, Damji, Campbell, \& Bryan, 2011; Rooshenas et al., 2015, p. 273). Policymakers recognize the necessity of complementing cautious investment in new technologies with strategies to reduce the use of unnecessary, ineffective, inefficient or harmful care that is currently used in practice (Chambers et al., 2017; Hodgetts, Elshaug, \& Hiller, 2012; Parkinson et al., 2015). The term 'disinvestment' refers to processes of 'partially or completely withdrawing health resources from existing healthcare practices, procedures, technologies or pharmaceuticals that are deemed to deliver little or no health gain for their costs, and thus do not represent efficient health resource allocation' (Elshaug, Hiller, Tunis, \& Moss, 2007). Disinvestment takes different forms ranging from the full withdrawal or substitution of services to the restriction of services due to inappropriateness of use or savings achieved through better organization of efficiency in care practices (Nuti, Vainieri, \& Bonini, 2010; Williams, Robinson, \& Dickinson, 2012). Reducing expenditure on 'low-value care'-i.e. 'interventions where the risk of harm or costs exceeds the likely benefit for a patient' (Chambers et al., 2017) - allows investment in higher valued care and in this way increases the efficiency of a health care system. Though low-value care practices seem obvious candidates for disinvestment, the withdrawal of resources from existing medical services unavoidably raises 'political and professional complexities' as it is associated with 'restrictions on clinical autonomy and patient choice' (Elshaug, Hiller, \& Moss, 2008).

It is commonly assumed that evidence of a medical treatment's (in)effectiveness of or data on inappropriate use can provide a factual, medical-technical base for thorny (dis)investment decisions (see e.g. Haines et al., 2014; Robinson, Williams, Dickinson, Freeman, \& Rumbold, 2012). One way to assess low-value care is by looking at routinely collected administrative data (Bhatia et al., 2015; Chalmers et al., 2018; Harris, Allen, et al., 2017; Scott \& Duckett, 2015). Administrative datasets containing information about health insurance claims, hospital admission, drug prescriptions et cetera provide population-level insights on service utilization (Chalmers et al., 2018). Low-value care can be found by comparing administrative data recording what actually happens in health care practice with, for example, the norms for practice as stated in (evidence-based) professional standards and guidelines. However, studies have shown that, despite the use of evidence to identify low-value care, disinvestment programs often face substantial social, political and cultural challenges (Elshaug et al., 2007; Hodgetts et al., 2012; Robinson et al., 2012; Rooshenas et al., 2015).

For example, disinvestment initiatives can encounter resistance to change due to clinicians' established medical training and practice paradigms (Elshaug et al., 2007, p. 4) and due to clinicians' unwillingness to engage in what is easily perceived as 'rationing' (Rooshenas et al., 2015). Disinvestment can lead to stakeholders' feelings of disempowerment and the idea that restrictive policies are 'imposed' on them by external agents, especially when stakeholders have no opportunities to 'influence draft policies' or are presented with 'near finalized' plans (Rooshenas et 
al., 2015, p. 278). The idea that external non-medical agencies have something to say about medical practice challenges clinicians' particular ownership of medical knowledge and is often associated with restrictions to clinical autonomy (Elshaug et al., 2008; Elshaug et al., 2007; Rooshenas et al., 2015). Effective disinvestment thus relies not only on sound technical reasoning, but also on an understanding of social values (Robinson et al., 2012), on local and national relationships (Daniels, Williams, Robinson, \& Spence, 2013) and on 'political will' (Elshaug et al., 2007). Literature on disinvestment therefore highlights the importance of inclusive dialogue, stakeholder involvement and transparency to create collaborative support for disinvestment initiatives (Daniels et al., 2013; Elshaug et al., 2008; Elshaug, Watt, Mundy, \& Willis, 2012; Garner \& Littlejohns, 2011; Hodgetts et al., 2012; Ibargoyen-Roteta, Gutierrez-Ibarluzea, \& Asua, 2010; Polisena et al., 2013; Robinson et al., 2012; Rooshenas et al., 2015).

While policymakers are generally aware of the fundamental importance that 'disinvestment is not perceived as a blunt, all-or-nothing instrument of rationing' (Elshaug et al., 2008, p. 7; Elshaug et al., 2007), both clinicians and policymakers often experience that they are 'operating within an environment of mistrust'(Rooshenas et al., 2015, p. 278). While policymakers fear that clinicians might be biased by potentially reduced revenues, clinicians, in their turn, fear that they might be wrongfully blamed that their practice is 'skewed by financial gain' (Rooshenas et al., 2015, p. 278). Mistrust and blame can hinder collaboration; inclusive dialogue and trust are seen as key aspects of successful disinvestment (Daniels et al., 2013; Elshaug et al., 2008; Elshaug et al., 2012; Garner \& Littlejohns, 2011; Hodgetts et al., 2012; Ibargoyen-Roteta et al., 2010; Polisena et al., 2013; Robinson et al., 2012; Rooshenas et al., 2015). In academic literature little has been written about effective practices of realizing 'trust' and (ultimately) collaborative support for disinvestment. This paper provides insights in this regard and examines how the epistemic practices of policymakers can help to establish trust and collaboration in disinvestment initiatives.

Drawing on ethnographic research in Dutch practice, we examined the epistemic practices of a group of Dutch policy advisors working on what was initially a disinvestment initiative within the context of the Dutch basic health insurance benefits package. The National Health Care Institute (Zorginstituut Nederland) is an independent governing agency that falls under the responsibility of the Ministry of Health. The Institute is tasked with the management of the basic benefits package in the Netherlands, and is lawfully responsible for the organization of public information about the quality of care. In 2014, this Institute introduced a program called Zinnige Zorg [hereafter translated as 'Appropriate Care'] that aimed to improve the quality of care funded from Dutch basic health insurance and save unnecessary public expenditure by identifying (and ultimately removing) ineffective and inappropriate procedures. The Institute's authoritative position in the Dutch health care system and its exclusive access to three national administrative datasets allowed the Institute to recognize low-value care practices and identify potential candidates for disinvestment. How did the Institute - in its epistemic practices - deal with the socio-political sensitivities associated with disinvestment?

We first introduce the Dutch health care system, the role of the National Health Care Institute within this system and the Appropriate Care program. We then elaborate on our theoretical 
background on epistemic practices and our research methods. In the results section, we analyze the Institute's epistemic practices in the Appropriate Care program and how the Institute dealt with trust and the socio-political sensitivities associated with disinvestment by means of their epistemic work.

\section{Dutch Health Insurance System and the National Health Care Institute}

In the Netherlands, standard basic health insurance is provided for all citizens by private health insurance companies. The 2006 Health Insurance Act (Zorgverzekeringswet, Zvw) obliges everyone who resides - or pays payroll tax - in the Netherlands to take out basic health care insurance from a private insurance company. Citizens can choose between competing health insurance companies during a yearly open enrollment period. Income-related subsidies make basic healthcare insurance affordable for all citizens. Insurers are obliged to accept enrollees regardless of their age or health condition, while a risk adjustment scheme compensates them for clients with predictably high medical expenses (Van de Ven \& Schut, 2009). Private insurance companies are expected to negotiate the prices, services, and quality of care with health care providers on behalf of their insured clients (Van de Ven \& Schut, 2008, p. 779).

The Minister of Health formally requests advice from the National Health Care Institute to specify treatments, drugs and medical aids that are reimbursed from the basic health insurance and those that are not. The Institute subjects major new drugs to health technology assessments (HTA), thereby systematically evaluating the drugs' (cost-) effectiveness, safety, ethical, social and financial issues, before coverage is considered. With regard to existing medical treatments and non-pharmaceutical interventions, the Institute issues authoritative standpoints whenever there is a lack of clarity amongst stakeholders about the coverage of a treatment, drug or medical aid.

\section{The Appropriate Care Program}

The Dutch government's Coalition Agreement in 2012 included a total cutback of approximately 300 million euros on basic health insurance, which was to be largely realized by a systematic screening of the basic benefits package. In 2013 the Minister of Health announced that this screening was to be carried out by the National Health Care Institute and that its goal was to stimulate appropriate use of the basic benefits package and identify potential savings (Schippers, 30 september 2013). In response, the National Health Care Institute set out to develop a new approach to package management: the Appropriate Care program.

'The main aspects of package management as currently implemented [HTA and reimbursement standpoints] are still important, but are merely preventing redundant, extra growth in costs [...]. The Appropriate Care (Zinnige Zorg) program was started in 2014 [...] to propose a new way of increasing the quality of care while economizing on costs; the key to this is identifying and reducing ineffective and/or unnecessary care' (Moerkamp, 1 maart 2017). 
A team at the National Health Care Institute started developing a program to screen the basic benefits package for low-value care by systematically combing through the package of provisions in a cyclical five-year process, addressing each of the 10 domains of the International Classification of Diseases (ICD-10).

Due to its unique position within the Dutch health care system (Helderman, De Kruijf, Verheij, \& Van Thiel, 2014), the Institute has exclusive access to three national administrative datasets: GIP data, DIS data and Vektis data. The GIP-database [Medicines and Medical Aids Information Project, GIP] contains prescription-related data on the use of drugs and medical aids. The DIS-database [Claims Information System, DIS], maintained by the Dutch Healthcare Authority (Nederlandse Zorgautoriteit), contains detailed information about all care trajectories carried out in hospitals, mental health organizations and rehabilitation centers. The Vektis-database (managed by the data specialist of Dutch health care insurers) covers a wider range of data including care for the elderly and paramedical care.

The Appropriate Care program consists of four phases in which low-value care is analyzed and tackled [see model 1]. First, in a 'Screening Phase', the Institute screens an ICD-10 domain for potentially low-value care. For indications of low-value care, the Institute consults different professional groups and sorts through professional media, patient-reported outcome measures (PROM), consultancy reports and scientific research on low-value care. The Institute also provisionally checks administrative data for matters that can indicate the inappropriate use of care, such as practice variations, massive increases or decreases in costs, extended length of hospital stay or high rates of adverse events, readmissions or re-operations. The screening phase results in a 'screening report', containing a set of topics for further research per ICD-10 domain. To date, six screenings have been finished: Diseases of the Circulatory System (ICD-10 IX100-199); Neoplasms (ICD-10 C00-D84); Diseases of the Respiratory System (ICD-10 X (J00-J99); Diseases of the Nervous System (ICD-10 VI (G00-G99)); Mental and Behavioral Disorders (ICD-10 V (F00-F99)); Diseases of the Genito-urinary System and Pregnancy, Childbirth and the Puerperium (ICD-10: XIV (N70-N98) and ICD-10: XV (O00-O99)).

Second, in an 'In-depth Analysis Phase', the Institute conducts in-depth analysis into the selected topics of low-value care. The Institute defines what is considered by the medical community to be 'appropriate' care for a diagnosis as specified, for example, in clinical guidelines and quality standards. It subsequently compares these norms to the actual care delivered in practice, by conducting a targeted analysis of the administrative data of the types of care registered and paid for in practice. This phase results in a 'room for improvement' report. In the 'room for improvement' report, the Institute proposes actions to improve care and it includes an estimation of the costs that can be saved in these practices. Furthermore, the Institute formulates agreements with health care professionals on how to improve the quality of those medical practices and ultimately save costs. So far, seven 'In-depth' analyses have been completed: care for Peripheral Artery Disease (PAD), care for chest pain (suspected stable angina pectoris), post-treatment surveillance of women treated for breast cancer, care for castration-refractory prostate carcinoma (CRPC), expensive oncolytics 
(appropriate care using expensive pharmaceuticals for metastatic renal cell carcinoma), end of life care for people with incurable intestinal cancer or lung cancer, care for arthrosis of the knee or hip.

Third, in the 'Implementation Phase', field parties - in dialogue with the National Health Care Institute - implement the health care improvement measures that were planned in the 'In-depth Analysis Phase'. Fourth, in the 'Monitoring Phase', the Institute monitors the progress made in the implementation of improvement measures and the results of these efforts. Seven topics are currently being implemented and monitored: PAD, chest pain, post-treatment surveillance of women treated for breast cancer, CRPR, expensive oncolytics, end of life care for incurable intestinal cancer or lung cancer, arthrosis in knee or hip. None of these topics have yet completed the Implementation and Monitoring Phases.

In order to identify low-value care, the Appropriate Care program works from the patients' perspective and uses 'Lean Thinking': following the patient as he/she goes through the health care system, aiming to identify value-added and non-value-added steps in the care process (see Institute for Healthcare Improvement, 2005). The focus in the Appropriate Care program is not on scraping services or technologies in their totality, but rather on targeting their (low-value) use for certain patients or in certain medical situations. Typically, the outcome of an Appropriate Care trajectory involves recommendations and tools for better guideline compliance, stepped care, shared decision-making, development of quality information (outcome indicators) and improved patient information (a similar approach to the 'Choosing Wisely' Campaign (Elshaug, McWilliams, \& Landon, 2013)). [See table 1 for two examples of Appropriate Care trajectories].

\section{Model 1 | Methodology of the Appropriate Care Program}

\section{Methodology}

Purpose: promoting appropriate care in the consulting room

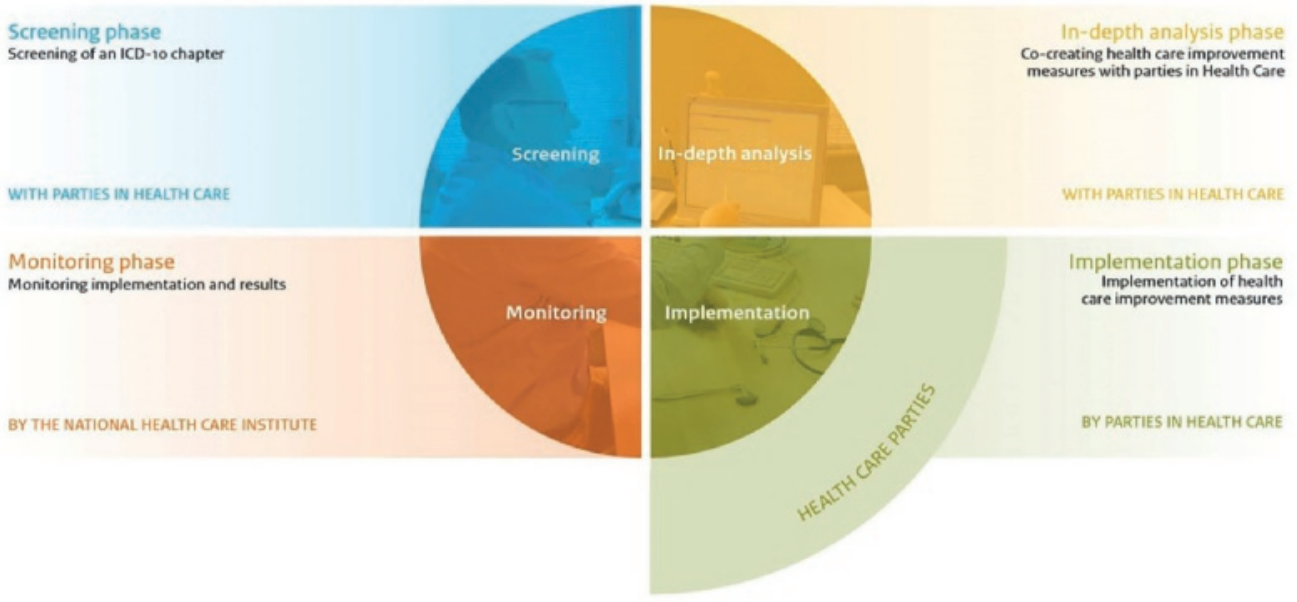



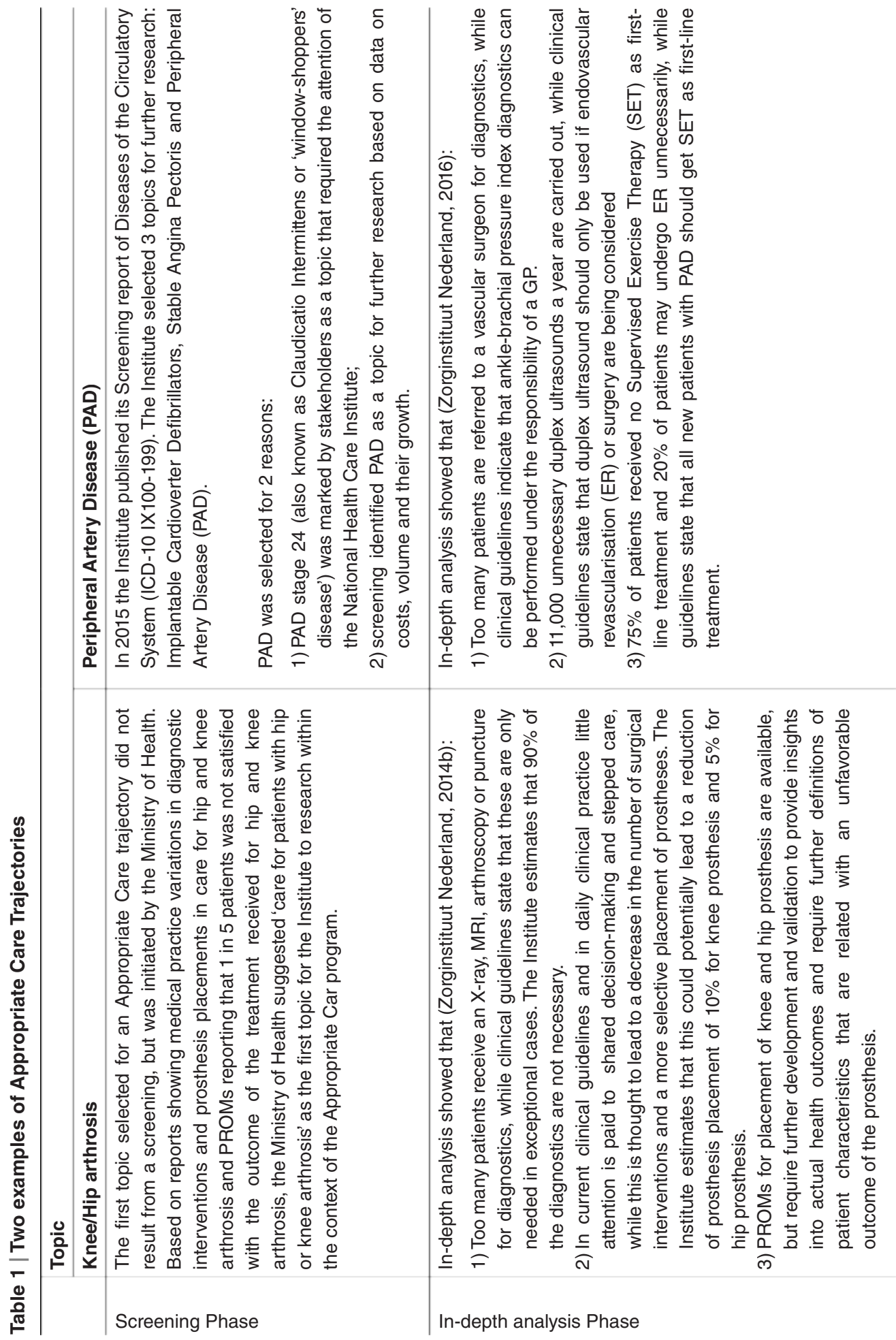


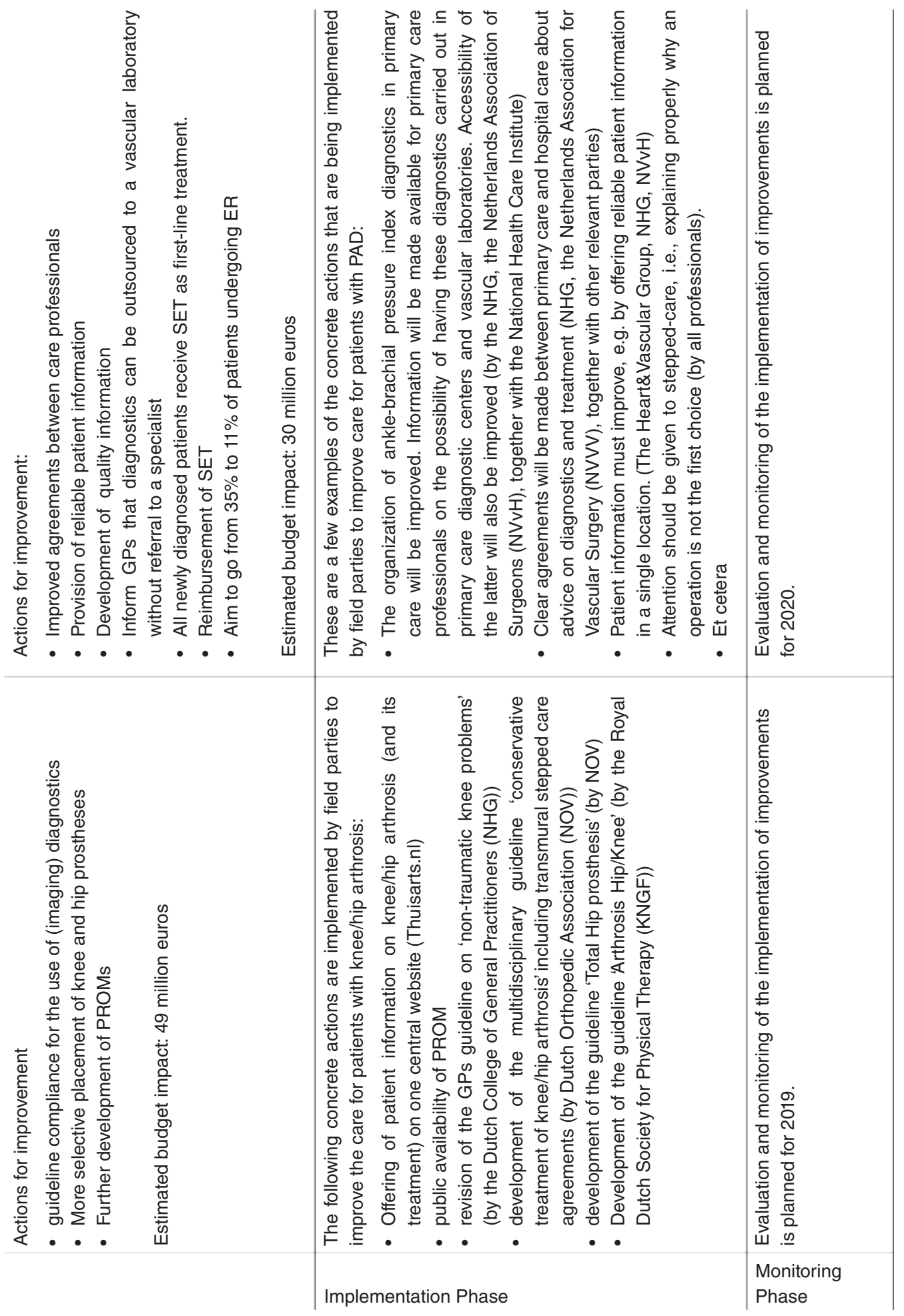




\section{Research Aim}

The widely reported social, political and cultural challenges to disinvestment (Elshaug et al., 2007; Hodgetts et al., 2012; Robinson et al., 2012; Rooshenas et al., 2015) hold particularly true for the Dutch context. Clinicians traditionally enjoy strong professional autonomy in the Netherlands and government interference in health care remains a sensitive issue (Bolt, 2015). Due to the corporatist structure under which the system of social health insurance operates, the Dutch have a consensus-building form of policymaking called 'poldering' that requires stakeholder engagement (Berg, Van der Grinten, \& Klazinga, 2004; Helderman et al., 2014). Though the National Health Care Institute is an authority in the field of social health insurance, at the same time, it occasionally encounters distrust from field parties. As manager of the basic benefits package, the Institute is easily perceived as an 'instrument of the Ministry of Health to cut health care expenditure' (Bolt, 2015 , p. 391). This paper explores how the Institute dealt with the delicate issue of balancing its own authoritative position in the field with clinicians' professional autonomy and the issues of (dis)trust that are typically associated with disinvestment efforts. This paper centers around the question of knowledge: how did the Institute deal with the socio-political sensitivities associated with disinvestment by means of their epistemic work?

\section{METHODS}

\section{Theoretical Background}

Science and Technology Studies (STS) (Felt, Fouché, Miller, \& Smith-Doerr, 2017) is a field of research that takes an empirical approach to studying knowledge in the daily practices of scientists, engineers and other professionals (Latour, 1987; Latour \& Woolgar, 1979). According to KnorrCetina, every place that produces knowledge claims is host to 'practices, arrangements and mechanisms' which - in that area of professional expertise - make up 'how we know what we know' (Knorr Cetina, 2007, p. 363). Such working methods, techniques or tools of knowing express an epistemic ideal of 'objectivity' in professional life (Porter, 1995). Ultimately, epistemic practices are related to how a profession makes itself publicly accountability for what it is licensed to do (Porter, 1995). Policymakers, too, have their own 'epistemic culture' (Knorr Cetina, 1999), and public accountability for policy choices is constituted in these particular epistemic practices (Porter, 1995).

This paper draws from four years of (etic) ethnographic research conducted at the National Health Care Institute. We studied the epistemic practices of 'how knowing is done' (de Laet, 2012, p. 429) at the National Health Care Institute. In particular, we looked at the Institute's practice of creating and warranting knowledge as a basis to justify policy decisions. Due to the public nature of the Institute, its knowledge work centers on the justification of decisions. What types of knowledge (e.g. experimental evidence, expertise, experience of doctors and patients) are used by the National Health Care Institute in its efforts to attain impartiality, objectivity and justice in policy decisions? 
In this paper we report on the epistemic practices of the Institute in the Appropriate Care program to understand how knowing was done and how legitimation and justification was achieved in the context of this program. As is customary in the field of STS, we refrain from taking a stance on what kind of knowledge should be used to attain impartiality, objectivity or justice in decisionmaking. Abstaining from taking a standpoint, allowed us to conduct systematic research into the way others ascribe meaning, truthfulness, political significance and moral weight to particular forms of knowing.

\section{Data Collection}

This paper is the result of a research project called 'Public Legitimation of Social Healthcare Insurance in the Modern Risk Society', funded and facilitated by the National Health Care Institute and independently executed by researchers of Maastricht University (see funding section at the end of this paper for specificities). From October 2013 to September 2017, the Institute's management provided the first author with an in-house desk (for 1-3 days a week), a digital workplace and access to archives to do intensive fieldwork within the Institute. The first author attended public and closed meetings of internal working groups (weekly), the executive board (two-weekly), as well as expert meetings of the Institute's advisory committees (Insured Package Advisory Committee, Quality Council, Scientific Advisory Board, Health Care Professions Committee), staff fora and informal lunches.

It is in daily practices that public accountability is 'done' by justifying and legitimizing public policy choices by drawing on specific working methods. We selected several concrete examples of the Institute's knowledge-intensive decision-making practices and our research focused progressively on these case-studies (see Moes, Houwaart, Delnoij, \& Horstman, 2017, 2018). In 2014 the National Health Care Institute introduced the new and pioneering Appropriate Care program. As this was a 'flagship' project for the Institute, we decided to focus part of the study on this program.

Between October 2013 and September 2017, empirical data were collected through in-depth conversations, document analysis, selective overt non-participant observation of meetings and formal interviews by the first author in three consecutive stages (as was the protocol for other case-studies (Moes et al., 2017, 2018)). First, to become familiar with the program, the first author held informal conversations with staff members involved and Institute directors. She delved into the Institute's archives to retrieve all written material referring to political, social and technical aspects of the epistemic work conducted in the context of the Appropriate Care program, including emails, minutes of meetings, official and internal documents. Second, to gain more direct experience with the epistemic practices, the first author attended three team meetings, seven executive board meetings, plus six meetings of advisory committees in which the program was discussed. Insights gained from in-depth conversations, document analysis and observations were recorded in daily field notes. Third, to triangulate our observations and documentary research, interviewees were selected through 'purposive' sampling of information-rich informants (Green \& Thorogood, 2004). 
We selected interviewees who held information about the political, social and technical aspects of the epistemic work conducted in the context of the Appropriate Care program. We carried out formal, semi-structured interviews with three key persons: the head of the Appropriate Care program, the program's data expert and a director of the Institute. Team members of the Appropriate Care program were interviewed together in a focus group setting that was held twice. For the focus group we selected team members who were involved explicitly in the epistemic work regarding the Appropriate Care program (technical analysis, data analysis, et cetera). All interviews were audiorecorded, after receiving the informants' consent, and transcribed in full.

\section{Data Analysis}

The analysis presented in this paper focuses on the epistemic work relating to the 'Screening Phase' and the 'In-depth Analysis Phase' of the Institute's Appropriate Care Program. We focus on these two phases because the working methods for these two phases were developed during our field work. Methods for the 'Implementation Phase' and the 'Evaluation Phase' were still in development when we were rounding off our fieldwork so they could not be included in our analysis. The data were analyzed as follows: the first (FM), second $(\mathrm{EH})$ and fourth $(\mathrm{KH})$ authors engaged in an iterative process of joint close reading of field notes, reports, policy documents, minutes and interview transcripts (that were manually coded and analyzed). The third author (DD) engaged in discussions of draft versions of this paper. The leading questions in the analysis were: What data, information and knowledge sources were used by the Institute in their effort to identify low-value care? How was the process of data analysis organized? Who was involved in formulating research questions and interpreting the results of these inquiries? How were data analyses that identified lowvalue care practices made public and actionable? In finding answers to these questions, we aimed to understand how the Institute dealt with socio-political sensitivities associated with disinvestment by means of their epistemic work. As a 'member-check', we sent a written version of the analysis presented in this paper to staff members involved and interviewees to test our analysis on them.

\section{RESULTS}

\section{Warranting Data Analysis by Clinical Experts}

The first focus group revealed that the basic idea of the Appropriate Care program was to identify low-value care by comparing what was regarded by the medical community as good practice (as specified for example in clinical guidelines and quality parameters) with care actually delivered in practice (for example by looking at administrative data on the treatments registered and paid for in practice). One of the participants in the focus group explained:

"I think that the real point of departure has always been, what do those who work in the field regard as good care? And where have they recorded this, e.g. in guidelines, or 
possibly in the quality parameters that they generally use? (...) and the second question is: is this how it is done in practice? (...) For example, we all feel that, before operating on claudicatio intermittens, you first determine whether physiotherapy helps, don't you? This is called 'running training'. Then you look at the [reimbursement] data: how many people have had the running training?" (focus group \#1).

If patients did not receive physiotherapy or 'running training' before undergoing an operation, this could be an indication of low-value care, because patients are unnecessarily subjected to invasive surgery.

However, our field notes about Appropriate Care program meetings showed that the technical analysis of administrative data was no easy task. To illustrate this, we show an excerpt from our field notes below. These field notes were taken at a meeting between the Appropriate Care program team and two external data consultants. During the meeting, the group looked at administrative data on the treatment of lung cancer and investigated the hypotheses that care for lung cancer patients in the follow-up treatment after initial surgery was inefficient, and thus, low-value care. The group tried to make sense of the surprisingly low number of lung cancer patients who were actually in follow-up trajectories. The first author recorded some of the questions that were raised with regard to the administrative data:

"the survival is only $20 \%$... maybe this explains the low numbers?... which patients should be selected? ...would it be a mistake to focus on those who underwent an operation? ... this dataset does not allow us to see who died... the number of people who do not return for a follow-up treatment - are they the ones who died?" (unpublished observation FM).

The Institute found that, in order to interpret the data accurately it was necessary to closely involve clinical experts who knew not only clinical guidelines, but also understood clinical practice as well as administrative practices. Therefore, the Institute hired clinical experts at the Institute to help conduct data analysis:

"We hire knowledge experts from the field who spend four days a week operating, and one day cooperating with us. In fact, we can learn an awful lot from them. [A clinical expert can do] a spot of brainstorming with us and he/she can demarcate our populations and say: 'well, if you are looking at this treatment it would be nonsensical not to look at that treatment as well..." (focus group \#1)

From our focus groups it became clear that the in-house availability of clinical expertise facilitated the Institute to carry out a better technical data analysis of low-value care. Focus group participants suggested that there was also an additional socio-political advantage to the close involvement of 
clinical experts. Data analysis performed only by non-medical parties - "people who have studied business administration" or "blue suits" (focus group \#1 and 2) - often lack the trust of field parties. Focus group participants suggested that the fact that data analysis was performed with the aid of clinical experts generated a form of trust that policymakers and data experts alone did not have.

\section{Epistemic Participation of Stakeholders}

The first topic that was investigated in the context of the Appropriate Care program was whether patients with arthrosis in hip and knee were receiving appropriate care. From our documentary research and interviews it became clear that the Ministry of Health suggested this topic to the Institute, based on PROMs showing that one out of five patients claimed no improvement after a hip or knee prosthesis and on a report of medical practice variations in treatment of knee and hip arthrosis (Zorginstituut Nederland, 2014a). One of our interviewees recalled: "it seemed like a really good topic to tackle immediately" (informant\#3).

However, according to interviews and focus groups, the Institute found that orthopedists were surprised that the Institute was investigating the appropriateness of care for knee and hip arthrosis as orthopedists had not played any significant part in selecting this topic for screening. Partly in response to this event, and partly because stakeholder involvement is typically part of the Institute's style of decision-making, the Institute decided to consult field parties systematically in the process of selecting research topics in the Screening Phase. In subsequent Appropriate Care trajectories, field parties had the opportunity to bring topics to the table that seemed important based on their experience and perspectives.

Not only the screening for topics of low-value care was done in negotiation with field parties, stakeholders were also involved in the subsequent epistemic work of designing relevant research questions. An Appropriate Care trajectory now starts with a 'kick-off' meeting that gathers representatives of all relevant parties at one table. The focus group revealed that stakeholders are involved in the epistemic practices of the Appropriate Care program right from day one:

"formulating the research question, in the sense of involving the parties as well, ... i.e., which questions need to be answered by the data. In other words, involving them up front, and then setting to work and finally checking the results with them" (focus group $\# 1)$.

From our interviews it became clear that the participation of stakeholders was both a way to include the knowledge of field parties in screening for low-value care, as well as a way to address issues of trust. An interviewee revealed that the participation of stakeholders was all about "having increasing confidence in one another" (informant\#1). In our field notes of an executive meeting, we recorded a board members saying on the matter of stakeholder participation that it is best "to involve them [field parties] in smaller steps and allow dominant choices to be partly theirs too" (unpublished observation FM). This did not mean that the Institute left choices, for example of screening topics, entirely up to stakeholders. Focus group participants explained: "where are the most important 
topics? In answering this, of course, we look at what impact we can have" (focus group\# 2), for example practices with a high volume of patients or high costs, as this would potentially yield the highest gains in health quality and save public costs. Working from the perspective of a public governing agency, the Institute does prefer some topics over others: "we introduce societal interests, so this gives us a strong position at the table" (focus group\#1).

\section{Creating an Epistemic Safe Space}

The use of national administrative data allowed the Institute to map care that was registered and paid for in practice. Our interview with the data expert revealed that these data allowed a wide range of analyses:

"You can in fact combine everything at hospital level.... or you can do it based on time: e.g. how many operations were carried out on that day? or you can do it at patient level: e.g. what happened to the patient over the course of time?... you can do an awful lot with this [these data]" (informant\#2).

Reimbursement data, however, is privacy-sensitive information. From our interviews it became clear that the Institute was very cautious in handling and publishing these data. The data expert commented that "care providers or hospitals allow you to see something of what is going on behind the scenes... which means they are to a degree relinquishing some of their power" (informant\#2). One of the lessons that the Institute learned in the context of the Appropriate Care program was that "handling data correctly" (informant\#2) was very important, especially when publishing data that could be traced back to specific institutions.

The data expert explained that, while it is broadly accepted "that you do not publish data on patients", still a topic of public debate is whether this is also the case for "publicly funded institutions" (informant\#2). As Dutch citizens spend so much public money on health care, it might be appropriate that everyone in society is actually allowed to see the aggregate result of "all those invoices on which we are all spending our [public] money" (informant\#2). The publication of e.g. data about practice variations can be relevant, furthermore, for both patients and insurers, as it conveys information about the quality of care provided in different hospitals. Despite the fact that it was interesting from a public (accountability) perspective to publish data on practice variations, the Institute never published hospital-specific or provider-specific information in any of its reports. Our interviews showed that the Institute actively avoided the publication of data that could be traced back to specific institutions in order to create a 'safe environment' for parties:

"you have to conduct the substantive discussion in a safe environment [...] If we were to immediately publish reports painting the full picture of everything that goes wrong, then the discussion would change entirely, as everyone would crawl back into their shell" (informant \#1) 
Our documentary research revealed that the professional media debated the Institute's decision not to publish medical practice variation data on care for cancer at an institutional level, and blamed the Institute for lack of transparency and listening too closely to the interests of care providers (Kiers, 2017). The National Health Care Institute, however, claimed that they did not have a mandate to publish institution-specific data (Tweede Kamer der Staten-Generaal, Vergaderjaar 2017-2018). Our interviews showed that, while the publication of institute-specific data could be a strong tool for holding professionals accountable, for the Institute it was important that things did not get personal in order to keep field parties involved. One interviewee commented: "if it isn't necessary... then we do not reveal information about care providers... [it is a matter of] balancing the power of communication versus [...] unnecessary disclosure" (informant\#2). In order to keep the stakeholders on board, the Institute conducted their epistemic work involving privacy-sensitive information about low-value care in an environment that was safe for those who were the object of that inquiry.

Our focus group reported that the Institute did organize multi-stakeholder meetings in which findings were discussed on a general level (i.e. not institute-specific). At these meetings professional groups started "asking one another questions" (focus group \#1). For example, when research showed that an 'awful lot of arthroscopies were carried out for arthritis':

"then patient [organizations] started saying: 'That's not a good thing at all, not if it's not doing anyone any good'... And, then the physiotherapists said: 'Yes, those patients are far better off going to a physiotherapist' [...] If you speak to the parties individually, then they could say: 'Yes, but GPs are too quick to refer, you shouldn't be talking to me, but to the GPs'... However, when they too are at the table, then you see that things are expressed differently and possibly also that people start saying: well, yes, perhaps we also played a role here..." (focus group \#1)

As such, professional groups were involved both in interpreting the study results and in formulating potential solutions for low-value care problems, without having to fear potential cutbacks or reputational damage for their own specific institutions.

\section{Shifting the Aim of Epistemic Work}

The Appropriate Care program was initiated after the Minister of Health announced that the National Health Care Institute would be carrying out a systematic screening of the benefits package in order to realize budget cuts in the context of social health insurance. This screening would identify lowvalue care practices currently covered by the basic benefits package. Our formal interviews and focus groups identified resistance to economizing or ministerial budget cuts on the part of those working at the Institute. The Institute did not want the Appropriate Care Program to be directly associated with disinvestment. In our field notes we recorded one of the Executive Board members expressing the following concern: 
"enormous pitfall in the entire trajectory... the link with economizing; although this is what the Minister requested... we do not carry out economization tasks, we look at quality and effectiveness" (unpublished observation FM)

From our interviews it became clear that the principle goal of those working for the Appropriate Care program was ensuring that patients received the right care at the right time in the right way. The idea was that this would save money in the long run (even though it might entail investment in the short term). Focus group participants revealed that "naturally, we do have to choose topics that we feel will eventually result in real economies..." (focus group \# 1). Yet, in their epistemic work - in formulating hypotheses, research questions and interpreting data analysis - the Institute put the emphasis on quality and safety concerns before economization. The focus group participants explained that, in the Appropriate Care Program: "we [have] always thought that if you promote quality, the euros would follow (...) the focus is on improving quality $(. .$.$) we weren't trying to remove$ provisions from the benefits package" (focus group \#1). The Institute treated economization not as the main goal of their epistemic work with regard to low-value care, but as a spin-off to organizing more efficient, high quality and safe health care.

From our observations, interviews and focus groups, it became clear that the reason to push the aim of economization to the background was also related to issues of trust. One interviewee revealed, for example, that talks with field parties always focused first on how to improve the efficiency, safety and quality of low-value care practices, and only thereafter "we chart what actual [financial] effect these [improvements] will have; and this ... is sensitive, because the parties involved fear that they will be short-changed" (informant\#3). In order to keep stakeholders on board in the Institute's effort to identify and tackle low-value care practices currently covered from basic health insurance, the Institute moved the goal of economization to the background in the Appropriate Care program.

\section{DISCUSSION}

In the Appropriate Care program (the systematic screening of the basic benefits package), the National Health Care Institute aimed to identify and ultimately eliminate low-value care practices that were currently covered from basic health insurance as well as potential underuse, and achieve savings by improving the organization of efficiency and quality in health care delivery. The Institute's authoritative position in the Dutch social health insurance system and its exclusive access to three national administrative datasets gave the Institute a unique position for locating low-value care on a national scale. Academic literature on disinvestment, however, shows that the right data and the authority to use them is not enough; disinvestment inevitably requires 'clinical leadership' (Robinson et al., 2012, p. 2391) and 'political will' on the part of the stakeholders (Elshaug et al., 2007). We examined how the Institute dealt with socio-political sensitivities associated with disinvestment by means of their epistemic work. 
We found, first of all, that the Institute hired clinical experts in-house to create technically solid data analyses that were warranted by clinical experts and therefore were expected to be considered trustworthy. Second, to avoid stakeholders' feelings of disempowerment and to improve technical analysis, the Institute developed a working method that engaged stakeholders in problem definition, formulating knowledge strategies and finding policy solutions. Third, the Institute created a safe space for deliberations about low-value care practices by actively avoiding the publication of information about low-value care that could be traced back to specific institutions. Fourth, the Institute shifted the goal of economization to the background and focused primarily on issues of safety and quality of care in its epistemic practices.

In summary, the Institute dealt with the socio-political sensitivities that are generally associated with such disinvestment initiatives by de-emphasizing the economization measure, de-politicizing the public deliberative space about low-value care practices, and by democratizing the epistemic practices to identify low-value care. Ultimately, this epistemic work facilitated a collaborative construction of problems relating to low-value care practices and their solutions.

\section{Limitations}

As the scope of our fieldwork allowed us to study only the 'Screening' and the 'In-depth Analysis' Phases, this research is a limited sample of the work performed in the Appropriate Care program. Our analysis of the epistemic practices of the program shows how the Institute manages to get field parties involved in identifying low-value care and in discussing potential solutions to tackle them. However, apart from stakeholders' involvement in the epistemic work of identifying low-value care and formulating problem solutions, successful disinvestment requires stakeholders' engagement in implementation and monitoring. International literature reports that there is still a lack of information about effective approaches and clear procedural tools for the implementation of disinvestment policy (Harris, Green, \& Elshaug, 2017; Hollingworth et al., 2015; Rooshenas et al., 2015). Unfortunately, because the 'Implementation' and 'Evaluation' phases were still in development at the time of our fieldwork, we cannot report on the procedural tools for implementation (and their effectiveness) in the context of the Appropriate Care program. Further research is required into the epistemic practices in all four phases of the program in order to learn how these epistemic practices contribute to the actual realization of disinvestment on low-value care.

\section{CONCLUDING REMARKS}

Routine administrative data are no longer a source that insurers, hospitals or medical professionals draw from to account for what they themselves have done; they have become a means by which the system has started to account for itself (cf. Freeman, 2002). In this paper we studied the use of administrative data by the Dutch National Health Care Institute to reduce unnecessary public expenditure by identifying and tackling low-value care currently covered from the basic benefits 
package. Our analysis of the Institute's Appropriate Care program shows how the epistemic effort to identify low-value care became a co-construction between policymakers, care providers, patients and insurers of problems of 'waste' in the Dutch social health insurance system. Whether or not disinvestment was actually achieved, the collective epistemic work performed within the Appropriate Care program gave cognitive, moral and political standing to the idea of 'waste' in public health expenditure.

Acknowledgements The authors gratefully acknowledge all research participants at the National Health Care Institute for their valuable contributions to our study. The authors also thank two reviewers for their inspiring feedback on an earlier draft of this paper. 
Abbreviations HTA: health technology assessment; ICD-10: International Classification of Diseases; GIP: Medicines and Medical Aids Information Project; DIS: Claims Information System; PROM: patient-reported outcome measures; PAD: Peripheral Artery Disease; CRPC: CastrationRefractory Prostate Cancer; GP: general practitioner; NHG: Dutch College of General Practitioners; NOV: Dutch Orthopedic Association; KNGF: Royal Dutch Society for Physical Therapy; ER: Endovascular Revascularization; SET: Supervised Exercise Therapy; NvVH: the Netherlands Association of Surgeons; NVVV: the Netherlands Association for Vascular Surgery; STS: Science and Technology Studies; FM: Floortje Moes; EH: Eddy Houwaart; KH: Klasien Horstman; DD: Diana Delnoij.

Competing interests The authors declare that they have no competing interests.

Ethics approval and consent to participate As this study did not involve research on patients no ethics committee approval was required under Dutch law. Neither the Dutch Medical Research Involving Human Subjects Act [WMO, Wet medisch-wetenschappelijk onderzoek met mensen] nor the University require ethics approval for the type of work conducted in this research. ${ }^{1}$ The study is based partly on documentary research, partly on interviews and observations. Management of the National Health Care Institute granted access to archives and to all public and closed meetings of internal working groups, the Institute's executive board, as well as expert meetings of advisory committees. All those attending these meetings were informed about the research and consented to our presence at the meetings. All interviewees were informed about the study beforehand and agreed to participate. We obtained permission from interviewees to quote from the interviews and obtained permission from the National Health Care Institute to cite from field notes. Interviewees and staff members of the Institute proof-read the paper, checked the case description and acknowledged its accuracy.

Availability of data and material The datasets used and/or analyzed during the current study are available from the corresponding author on reasonable request.

Funding The research project was funded by the Dutch National Health Care Institute. The Institute as a funding source had no involvement in the analytical content of the paper (or any of the other papers published within the context of this research project). DD, who is affiliated with the National Health Care Institute, approved the research design of the project, helped to acquire access for the field work at the National Health Care Institute, and critically read and discussed data analysis and drafts of this article. Relevant staff members of the Institute were involved in checking the accuracy of the case description. After having proof-read the paper, the National Health Care Institute acknowledged the accuracy of the case description.

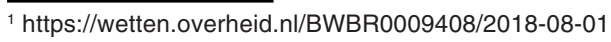


Authors' contributions We hereby confirm that all authors made substantial contributions to the manuscript. $\mathrm{FM}, \mathrm{EH}$, and $\mathrm{KH}$ contributed to the conception and design, acquisition of data, analysis and interpretation of data, and in drafting of the article and revising it critically. DD was also involved during all stages of the research: DD approved the research design, helped to acquire access for the field work at the National Health Care Institute, and critically read and discussed data analysis and drafts of the article. All authors gave, furthermore, final approval of the version to be published and agree to be accountable for all aspects of the work and ensure that questions relating to the accuracy or integrity of any part of the work are appropriately investigated and resolved. 


\section{REFERENCES}

Berg, M., Van der Grinten, T., \& Klazinga, N. S. (2004). Technology assessment, priority setting, and appropriate care in Dutch health care. International Journal of Technology Assessment in Health Care, 20(1), 35-43.

Bhatia, R., Levinson, W., Shortt, S., Pendrith, C., Fric-Shamji, E., Kallewaard, M., . . Forde, I. (2015). Measuring the effect of Choosing Wisely: an integrated framework to assess campaign impact on low-value care. BMJ Quality \& Safety, 24(8), 523-531.

Bolt, T. (2015). A doctor's order: the Dutch case of evidence-based medicine (1970-2015). AntwerpenApeldoorn: Garant.

Chalmers, K., Badgery-Parker, T., Pearson, S.-A., Brett, J., Scott, I., \& Elshaug, A. (2018). Developing indicators for measuring low-value care: mapping Choosing Wisely recommendations to hospital data. BMC research notes, 11(1), 163.

Chambers, J., Salem, M., D’Cruz, B., Subedi, P., Kamal-Bahl, S., \& Neumann, P. (2017). A Review of Empirical Analyses of Disinvestment Initiatives. Value in Health, 20(7), 909-918.

Daniels, T., Williams, I., Robinson, S., \& Spence, K. (2013). Tackling disinvestment in health care services. Journal of Health Organization and Management, 27(6), 762-780.

de Laet, M. (2012). Anthropology as Social Epistemology? Social Epistemology, 26(3-4), 419-433.

Elshaug, A., Hiller, J., \& Moss, J. (2008). Exploring policy-makers' perspectives on disinvestment from ineffective healthcare practices. International Journal of Technology Assessment in Health Care, 24(1), 1-9.

Elshaug, A., Hiller, J., Tunis, S., \& Moss, J. (2007). Challenges in Australian policy processes for disinvestment from existing, ineffective health care practices. Aust New Zealand Health Policy.

Elshaug, A., McWilliams, J., \& Landon, B. (2013). The value of low-value lists. JAMA, 309(8), 775-776.

Elshaug, A., Watt, A., Mundy, L., \& Willis, C. (2012). Over 150 potentially low-value health care practices: an Australian study. Medical Journal of Australia, 197(10), 556-560.

Felt, U., Fouché, R., Miller, C., \& Smith-Doerr, L. (2017). The handbook of science and technology studies. Cambridge; Mass: MIT Press.

Freeman, T. (2002). Using performance indicators to improve health care quality in the public sector: a review of the literature. Health Services Management Research, 15(2), 126-137.

Garner, S., \& Littlejohns, P. (2011). Disinvestment from low value clinical interventions: NICEly done? BMJ, 343, d4519.

Green, J., \& Thorogood, N. (2004). Qualitative Methods for Health Research. London: SAGE Publication.

Haines, T., O’Brien, L., McDermott, F., Markham, D., Mitchell, D., Watterson, D., \& Skinner, E. (2014). A novel research design can aid disinvestment from existing health technologies with uncertain effectiveness, costeffectiveness, and/or safety. Journal of Clinical Epidemiology, 67(2), 144-151.

Harris, C., Allen, K., King, R., Ramsey, W., Kelly, C., \& Thiagarajan, M. (2017). Sustainability in Health care by Allocating Resources Effectively (SHARE) 2: Identifying opportunities for disinvestment in a local healthcare setting. BMC Health Services Research, 17(1), 328.

Harris, C., Green, S., \& Elshaug, A. (2017). Sustainability in Health care by Allocating Resources Effectively (SHARE) 10: operationalising disinvestment in a conceptual framework for resource allocation. BMC Health Services Research, 17(1), 632. 
Helderman, J., De Kruijf, J., Verheij, J., \& Van Thiel, S. (2014). Dike-Reeve of the health care polder. A politicalsociological analysis of the realisation of the National Health Care Institute against a backdrop of a changing policy agenda and changing political-administrative and societal relations. Diemen: Zorginstituut Nederland.

Hodgetts, K., Elshaug, A., \& Hiller, J. (2012). What counts and how to count it: Physicians constructions of evidence in a disinvestment context. Social science \& medicine, 75(12), 2191-2199.

Hollingworth, W., Rooshenas, L., Busby, J., Hine, C., Badrinath, P., Whiting, P., . . Donovan, J. (2015). Using clinical practice variations as a method for commissioners and clinicians to identify and prioritise opportunities for disinvestment in health care: a cross-sectional study, systematic reviews and qualitative study. Health Services and Delivery Research, 3(13), 1-172.

Ibargoyen-Roteta, N., Gutierrez-lbarluzea, I., \& Asua, J. (2010). Guiding the process of health technology disinvestment. Health Policy, 98(2-3), 218-226.

Institute for Healthcare Improvement. (2005). Going Lean in Health Care IHI Innovation Series white paper. Cambridge, Mass.: Institute for Healthcare Improvement (IHI).

Kiers, B. (2017, 18 December 2017). Ziekenhuizen behandelen intensief door bij levenseinde. Zorgvisie.

Knorr Cetina, K. (1999). Epistemic Cultures: How the Sciences Make Knowledge. Cambridge, Mass.: Harvard University Press.

Knorr Cetina, K. (2007). Culture in global knowledge societies: knowledge cultures and epistemic cultures. Interdisciplinary Science Reviews, 32(4), 361-375.

Latour, B. (1987). Science in Action: how to follow scientists and engineers through society. Cambridge, Mass.: Harvard University Press.

Latour, B., \& Woolgar, S. (1979). Laboratory Life: the construction of scientific facts. Princeton (N.J.): Princeton University Press.

Mitton, C., Dionne, F., Damji, R., Campbell, D., \& Bryan, S. (2011). Difficult decisions in times of constraint: Criteria based Resource Allocation in the Vancouver Coastal Health Authority. BMC Health Services Research, 11(1), 169.

Moerkamp, A. (1 maart 2017). Zinnige Zorg naar de praktijk. (2017009954). Diemen: Zorginstituut Nederland, .

Moes, F., Houwaart, E., Delnoij, D., \& Horstman, K. (2017). Contested evidence: a Dutch reimbursement decision taken to court Health economics, policy, and law, 12(3), 325-344.

Moes, F., Houwaart, E., Delnoij, D., \& Horstman, K. (2018). "Strangers in the ER": Quality indicators and third party interference in Dutch emergency care. Journal of Evaluation in Clinical Practice, 1-8.

Nuti, S., Vainieri, M., \& Bonini, A. (2010). Disinvestment for re-allocation: A process to identify priorities in healthcare. Health Policy, 95(2), 137-143.

Parkinson, B., Sermet, C., Clement, F., Crausaz, S., Godman, B., Garner, S., ... Elshaug, A. (2015). Disinvestment and Value-Based Purchasing Strategies for Pharmaceuticals: An International Review. PharmacoEconomics, 33(9), 905-924.

Polisena, J., Clifford, T., Elshaug, A., Mitton, C., Russell, E., \& Skidmore, B. (2013). Case studies that illustrate disinvestment and resource allocation decision-making processes in health care: a systematic review. International Journal of Technology Assessment in Health Care, 29(2), 174-184.

Porter, T. (1995). Trust in Numbers: the Pursuit of Objectivity in Science and Public Life. Princeton: Princeton University Press. 
Robinson, S., Williams, I., Dickinson, H., Freeman, T., \& Rumbold, B. (2012). Priority-setting and rationing in healthcare: Evidence from the English experience. Social science \& medicine, 75(12), 2386-2393.

Rooshenas, L., Owen-Smith, A., Hollingworth, W., Badrinath, P., Beynon, C., \& Donovan, J. (2015). "I won't call it rationing...": An ethnographic study of healthcare disinvestment in theory and practice. Social science \& medicine, 128, 273-281.

Schippers, E. I. (30 september 2013). Buitenhof-oproep, aanpak doorlichting pakket en reactie op twee CVZrapporten: Geneeskundige Geestelijke Gezondheidszorg, deel 2 en Kosteneffectiviteit (154224-110833-Z). Den Haag.

Scott, I., \& Duckett, S. (2015). In search of professional consensus in defining and reducing low-value care. The Medical journal of Australia, 203(4), 179-181.

Tweede Kamer der Staten-Generaal. (Vergaderjaar 2017-2018). Antwoord op vragen van de leden Pia Dijkstra en Bergkamp over het bericht 'Ziekenhuizen behandelen intensief door bij levenseinde' (ah-tk-20172018-1287). 's-Gravenhage: Tweede Kamer der Staten-Generaal Retrieved from https://zoek.officielebekendmakingen.nl/ ah-tk-20172018-1287.html.

Van de Ven, W., \& Schut, F. (2008). Universal mandatory health insurance in the Netherlands: A model for the United States? Health Affairs, 27(3), 771-781.

Van de Ven, W., \& Schut, F. (2009). Managed competition in the Netherlands: Still work-in-progress. Health Economics, 18(3), 253-255.

Williams, I., Robinson, S., \& Dickinson, H. (2012). Rationing in Health Care the theory and practice of priority setting. Bristol: Policy.

Zorginstituut Nederland. (2014a). Concept analyse voor consultatie zorg bij artrose van knie en heup. ICD: 500-T88. Diemen: Zorginstituut Nederland.

Zorginstituut Nederland. (2014b). Verbetersignalement zorg bij artrose van knie en heup, ICD-10: XIII M 16, 17 (P. Z. Zorg, Trans.). Diemen: Zorginstituut Nederland.

Zorginstituut Nederland. (2016). Room for Improvement report: Peripheral arterial disease. Diemen: Zorginstituut Nederland. 




\section{Chapter 4}

\section{'Epistemic Injustice' in Knowledge-intensive Policymaking}

Based on: Moes, F., Houwaart, E., Delnoij, D., \& Horstman, K. 'Questions regarding 'Epistemic Injustice' in knowledge-intensive policymaking: two examples from Dutch health insurance policy' 


\section{ABSTRACT}

This paper examines the usefulness and limits of the concept of 'epistemic injustice' in the context of policy practices. Miranda Fricker's concept of 'epistemic injustice' refers to a wrong done to a person specifically in their capacity as a knower. Applying the idea of epistemic injustice to healthcare, some have argued that Evidence-based Medicine (EBM) is liable to generate epistemic injustice because its focus on quantitative population-based research privileges quantitative types of evidence and styles of articulating testimonies and - as a result- potentially undervalues the qualitative testimonies of doctors and patients. How is knowledge-intensive policymaking intertwined with questions of 'epistemic injustice'? This paper, drawing from ethnographic research conducted at the National Health Care Institute, analyzes two cases of EBM-inspired policy practices in Dutch social health insurance: 1) the use of the principles of EBM in making a public reimbursement decision, and 2) private insurers' use of quantitative performance indicators for the practice of selective contracting on the Dutch healthcare market. While the concept of 'epistemic injustice' misses some key processes involved in understanding how 'knowing gets done' in public policy, our analysis of these two cases from this perspective, sheds new light on priority-setting processes. Patients or medical professionals who are not duly recognized as credible and intelligible epistemic agents, subsequently, lack the social power to influence priority-setting practices. They are thus not merely frustrated in their epistemic capacity to be heard and make themselves understood, they are potentially deprived of a fair share in our collective financial and medical resources. If we fail to recognize inequalities in credibility and intelligibility between diverse groups of knowers, there is a chance that these latent epistemic inequalities are being reproduced in our system of health insurance and our ways of distributing healthcare provisions. 


\section{INTRODUCTION}

Evidence-based Medicine (EBM) gained strong traction in clinical practice amid growing recognition of 'the vagaries of clinical experience' (Armstrong, 2002, p. 1772) and the inadequacies of knowledge based on experience alone. The original idea behind EBM was to teach doctors to critically consume medical literature, emphasizing the use of epidemiological quantitative, experimental evidence in decision-making (Evidence-Based Medicine Working Group, 1992; Guyatt et al., 2008; Guyatt et al., 1995). Originally designed for clinical practice, the principles behind EBM - 'the conscientious, explicit, and judicious use of current best evidence' in medical decision-making (Sackett, Rosenberg, Muir Gray, Haynes, \& Richardson, 1996) - are now also used in health policy practices. Successes of EBM in improving modern healthcare have been widely acclaimed both by proponents and critics of EBM. A perennial question, however, remains: does the 'scientific' rule-based reasoning that underpins EBM sufficiently accommodate the complex and context-dependent reasoning required in clinical decisions (Greenhalgh, 2002) and in public decision-making (Dobrow, Goel, \& Upshur, 2004; Greenhalgh \& Russell, 2009)?

The term 'epistemic injustice', introduced by Miranda Fricker in her book 'Epistemic Injustice: Power and Ethics of Knowing', refers to 'a wrong done' to a person or group of people 'specifically in their capacity as a knower' (Fricker, 2007, p. 1). Applying the idea of epistemic injustice to healthcare, Carel and Kidd suggested that 'the structures of contemporary healthcare practice encourage epistemic injustice because they privilege certain styles of articulating testimonies, certain forms of evidence, and certain ways of presenting and sharing knowledge' (Carel \& Kidd, 2014, p. 530). Some have argued that EBM and its focus on quantitative population-based research unintentionally undervalues both doctors and patients 'as 'knowers' capable of making judgements outside the confines of epidemiological evidence' (Deaton \& Cartwright, 2018; Ghinea, Lipworth, \& Kerridge, 2015, p. 28; Greenhalgh, 2002; Greenhalgh, Snow, Ryan, Rees, \& Salisbury, 2015). While the founding fathers claim that EBM entails the integration of the best external evidence with individual clinical expertise and patients' choice (Sackett et al., 1996), critics have long been concerned that EBM's focus on epidemiological evidence and a formulaic decision-making model nonetheless undervalues situated clinical expertise of doctors and patient experiences and preferences (Greenhalgh et al., 2015; Lambert, 2006).

Inquiries into epistemic injustice in healthcare typically focus on the vulnerability of patients in clinical settings (Carel \& Kidd, 2014; Kidd \& Carel, 2017). However, the question of epistemic injustice is equally relevant when making knowledge-intensive policy decisions. In response to claims of a disengaged and disillusioned citizenry, policymakers have been experimenting with deliberative spaces to engage citizens more directly in setting the direction for policy decisions (Dieleman, 2015). Concerns are raised, however, about the 'epistemological status' that is actually accredited to public knowledge (Irwin, 2006, p. 315), and whether a public voice is (unintentionally) being 'made' or 'wrongly appropriated' in such 'formalized mechanisms of voice' (Felt \& Fochler, 2010; Irwin, 2006; Michael, 2012, p. 541). In the context of healthcare policy, it has been argued 
that patients do not always have the epistemic resources or capacity to 'effectively make use of' the possibilities offered to participate in formal decision-making procedures, and that procedures are not always designed to enable 'more than symbolic participation' (Peeters, Delnoij, \& Friele, 2014, p. 60).

Substantive inclusion requires that all parties are credibly understood. Fricker's work makes us aware that stakeholders' ability to participate meaningfully in public deliberation is dependent on their credibility and intelligibility (Fricker, 2007). While the concept of epistemic injustice has been widely applied in various academic fields for analyzing interpersonal relationships, few scholars have explored what the application of epistemic injustice to the public, political space would look like (Dieleman, 2015). In this paper we return to two earlier published case-studies of knowledgeintensive, EBM-inspired policy practices in Dutch health insurance (Moes, Houwaart, Delnoij, \& Horstman, 2017, 2018). We study whether diverse groups of knowers participate equally and meaningfully as epistemic agents in these public decision-making processes. On the basis of this analysis, we discuss the usefulness as well as the limits of the concept epistemic injustice in the context of policy practices.

\section{THE CONCEPT OF EPISTEMIC INJUSTICE}

The concept of epistemic (in)justice refers to the question of whether parties are duly recognized 'in their capacity as knower' and have a fair share of our 'collective interpretative resources' at hand in order to manifest what they know (Fricker, 2007, p. 1). Fricker identifies two types of epistemic injustice (Fricker, 2007). The first is 'testimonial injustice', which occurs when prejudice causes a hearer to give a 'deflated level of credibility' to a speaker's words (Fricker, 2007, p. 1). As an example of testimonial injustice, drawing from Harper Lee's book 'To Kill a Mockingbird', Fricker cites jurors' failure to believe the testimony of Tom Robinson - a black man in Maycomb County, Alabama, USA in 1935 - because they are prejudiced against black people (Fricker, 2007, p. 23). When someone lacks credibility, 'their assertions are not accepted by those to whom they are directed, and they are treated as lacking what is required to be a reliable informant' (Hookway, 2010, p. 152). This is considered a form of injustice, because this person is then unable to perform certain social and political tasks that demand credibility. According to Fricker, someone's status as epistemic agent affects his/her 'social power', which she defines as the 'capacity we have as social agents to influence how things go in the social world' (Fricker, 2007, p. 9).

A second form of epistemic injustice that inhibits social power is 'hermeneutical', referring to 'a gap in collective interpretive resources [that] puts someone at an unfair disadvantage when it comes to making sense of their social experiences' (Fricker, 2007, p. 1). While in 'testimonial' injustice someone is wronged in their capacity as 'a giver of knowledge' (Fricker, 2007, p. 7) and suffers a 'credibility deficit' (Coady, 2010, p. 110), in 'hermeneutical' injustice someone is wronged in their capacity as 'a subject of social understanding' (Fricker, 2007, p. 7) and suffers an 'intelligibility deficit' (Coady, 2010, p. 110). As an example, Fricker mentions Susan Brownmiller's book 'In Our Time: Memoir of a Revolution' in which she recounts the situation of Carmita Wood, 'a women 
who suffers sexual harassment prior to the time when we had this critical concept, so she cannot properly comprehend her own experience, let alone render it communicatively intelligible to others' (Fricker, 2007, p. 1). This is considered a form of injustice, because someone who cannot make $\mathrm{him} /$ herself understood is unable to perform social and political tasks that demand their intelligible contribution. Someone who suffers a lack of conceptual resources to make him/herself understood, is unable to effectively participate in 'practices through which social meanings are generated' (Fricker, 2007, p. 6).

According to Fricker we can (and should) fight epistemic injustice by cultivating the virtues of testimonial and hermeneutic justice. This is done by tracing instances of epistemic injustice and guarding against prejudice in assessing and processing the testimonies of others (Fricker, 2007). Critics, however, have argued that the 'virtue' of epistemic justice cannot reasonably be required (Alcoff, 2010; Langton, 2010; Sherman, 2016). As one critic put it:

'in the contemporary world, we are all constantly bombarded with testimony, from a variety of sources... We have limited evidence about many of these sources... Given the pressure of time, and the barrage of testimony, it is nearly impossible for us to make credibility judgments that match the evidence in all such cases. That is more than can be reasonably expected of us' (Maitra, 2010, p. 199).

According to these authors, Fricker's account lacks an explanation of who is responsible (in our private, professional, public or political lives?) - and when (in what situation?) - for taking on the role of the virtuous listener (Dieleman, 2015; Maitra, 2010). In this paper we explore these questions as we examine the relevance of the concept of 'epistemic justice' in the context of public decisionmaking.

\section{METHOD}

This paper draws from ethnographic research conducted at the National Health Care Institute (Zorginstituut Nederland), a public knowledge institution that plays a central role in the Dutch health insurance system and is responsible for making priority-setting decisions in the context of the basic benefits package and plays a key role managing the quality of care.

We studied 'how knowing is done' (de Laet, 2012, p. 429) at the National Health Care Institute, its 'epistemic culture' (Knorr Cetina, 2007). In particular, we looked at the Institute's practice of creating and warranting knowledge as a basis to justify policy decisions. Due to the public nature of the Institute, its knowledge work centers on the justification of decisions. We studied what types of knowledge (e.g. experimental evidence, expertise, experience of doctors and patients) the National Health Care Institute employs in its efforts to attain impartiality, objectivity and justice in policy decisions. 
From October 2013 to September 2017, the Institute's management provided the first author with an in-house desk, a digital workplace and access to archives to do intensive fieldwork within the Institute. The first author attended public and closed meetings of internal working groups, the executive board, as well as expert meetings of the Institute's advising committees, staff fora and informal lunches.

Taking a case-study approach, we selected several real-life struggles of the Institute in their knowledge-intensive decision-making processes and our research focused progressively on these case-studies. Two of the case-studies concerned 'struggles' in which specific types of knowledge or evidence supporting policy decisions were publicly contested as parties felt that their knowledge was not sufficiently recognized, their voices unheard or that they had not been taken seriously in the policymaking process. We selected these two cases to analyze the issue of 'epistemic injustice' in policymaking processes.

Each case was researched in the same consecutive stages: 1) familiarization with the case through informal conversations with staff and preliminary observations in meetings and fora; 2) supplementary data-gathering through document analysis (emails, minutes, internal and official documents, media articles) and selective overt non-participant observation of relevant meetings; and 3 ) in-depth research and triangulation through interviews with information-rich informants whom we selected through 'purposive' sampling (Green \& Thorogood, 2004).

The leading question in analyzing the two case-studies from the perspective of 'epistemic injustice' was: were parties duly recognized 'in their capacity as knower' and did they have a fair share of our 'collective interpretative resources' at hand in order to participate meaningfully in these knowledge-intensive decision-making processes? No additional data were gathered for the current paper, as we used available research archives from two earlier published cases (Moes et al., 2017, 2018). We do present previously unpublished material from the existing data set. As a 'membercheck', we sent a written version of the analysis to staff-members and directors involved, to test our analysis with them.

\section{QUESTIONS REGARDING EPISTEMIC INJUSTICE IN REIMBURSEMENT DECISIONS}

In the Netherlands, private health insurance companies provide standard basic health insurance for all citizens. The 2006 Health Insurance Act (Zorgverzekeringswet, Zvw) obliges everybody who resides - or pays payroll tax - in the Netherlands to take out basic healthcare insurance from a private insurance company. Income-related subsidies make basic healthcare insurance affordable for all citizens. Insurers are obliged to accept enrollees regardless of their age or health condition; a risk adjustment scheme compensates them for clients with predictably high medical expenses (Van de Ven \& Schut, 2009). Competition was introduced on two levels. First, citizens can choose between competing health insurance companies during a yearly open enrollment period. Second, 
private insurance companies are expected to negotiate the prices, services, and quality of care on behalf of their insured clients (Van de Ven \& Schut, 2008, p. 779).

The National Health Care Institute is tasked with managing the basic benefits package. The Minister of Health formally requests advice from the Institute on the medical-technical content of care that can be reimbursed from the basic health insurance. The Institute subjects major new drugs to cost-effectiveness analysis before coverage is considered (Staal, Heymans, Ligtenberg, Derksen, \& Couwenbergh, 2015). Such evaluations avoid the automatic inclusion of new drugs for intramural patients in the benefits package (Helderman, De Kruijf, Verheij, \& Van Thiel, 2014, pp. 23-25). The Institute provides authoritative standpoints on existing medical treatments and nonpharmaceutical interventions when healthcare professionals lack clarity about the reimbursement of these treatments. The first case-study is about one of these reimbursement decisions (Moes et al., 2017).

\section{The Case}

In 2013, the National Health Care Institute issued a reimbursement standpoint that bladder instillations with chondroitin sulphate or hyaluronic acid should no longer be covered by the basic health insurance, as there was no quantified proof of the treatment's effectiveness. Bladder instillations are a medical treatment used for various bladder conditions, including bladder cancer, interstitial cystitis and painful bladder syndrome. During treatment a fine tube (catheter) is inserted into the bladder, the bladder is then filled with a solution (including a pharmacological cocktail of e.g. chondroitin sulphate or hyaluronic acid) and after a period of time the bladder is drained.

To arrive at a positive reimbursement decision, the Institute requires conclusive proof of a treatment's effectiveness (College voor Zorgverzekeringen, 2007). The Institute uses the principles of EBM to gauge effectiveness, meaning that systematic reviews and double-blinded randomizedcontrolled trials (RCTs) are deemed to provide more objective information about treatment effectiveness than comparative or non-comparative research or expert opinions (College voor Zorgverzekeringen, 2007, pp. 8-9). Two double-blind RCTs showed improvements after treatment with bladder instillations with chondroitin sulphate or hyaluronic acid, but the difference with the placebo group was not statistically significant (College voor Zorgverzekeringen, 2013a, p. 11). The Institute therefore concluded that bladder instillations did not fulfil the reimbursement criteria as there was no quantitative proof of its effectiveness.

The Patients' Association for Interstitial Cystitis (ICP) sued the National Health Care Institute for misconduct against interstitial cystitis patients. Interstitial Cystitis (IC) refers to a spectrum of urological symptoms characterized by (chronic) pelvic, bladder and urethral pain, persistent urge to void or increased urinary frequency, as well as irritative voiding symptoms (Shao, Shen, Rui, \& Zhou, 2010). Some IC-patients claim to benefit from bladder instillations with chondroitin sulphate or hyaluronic acid. Also, urologists who frequently use these bladder instillations claim that their experience with this treatment is often positive. Urologists' professional organization, the Dutch Urological Association (NVU), agreed with the Institute that 'at the moment there is 
insufficient reliable research' that provided quantitative evidence of this treatment's positive effect, but stressed that the two RCTs did 'NOT prove that the washes are NOT effective' (College voor Zorgverzekeringen, 2013a, p. 21 emphasis in original).

The ICP's lawyer stated before the court: "I do not contradict that the efficacy of a treatment is a prerequisite for being eligible for inclusion in the insured benefits package" (hearing\#30042014). In making sense of this treatment's effectiveness, however, the lawyer argued that the EBM-method attributed too little credibility to the experience of doctors and patients: "what everyday practice thinks about something has too little evidential value in the EBM-method" (hearing\#30042014). Next, we examine the issue of testimonial injustice: were patients and urologists, indeed, insufficiently recognized in their capacity as knower?

\section{Testimonial (in)justice}

The ICP's lawyer introduced several patient testimonies about the experienced effect of bladder instillations with chondroitin sulphate or hyaluronic acid. A patient stated, for example, as follows:

'After a very tumultuous period lasting some months, my bladder has settled down as a result of the bladder instillations with hyaluronic acid. Because of the bladder instillations, my bladder hasn't suffered a 'flare-up' in almost a year' (exhibit 17 \#1)

While the Institute did not deny that individual IC patients might benefit from treatment with bladder instillations, they did not take patient testimonials into account. According to the principles of EBM, personal accounts do not suffice to establish a treatment's effectiveness and whether it 'works' for the average patient, especially in the case of IC. A staff member explained: "Some of the patients have a functional syndrome... and these are subjective outcome measures that you examine, i.e., improvement or reduction in symptoms. We were therefore explicit in saying 'we want randomized studies and not case series"' (notes\#03032014). In addition, the symptoms of IC fluctuate over time, which makes it even more difficult to attribute changes in symptoms to specific interventions. The Institute therefore decided:

'As interstitial cystitis is a disorder that is characterized by a fluctuating course and there is no standard treatment, randomized studies are needed in which bladder instillation with washes containing chondroitin sulphate and/or hyaluronic acid is compared with placebo washes. As the symptoms and outcome parameters are subjective, doubleblind studies are preferred' (College voor Zorgverzekeringen, 2013a, p. 10).

Double-blind and placebo-controlled RCTs keep both patient and doctor in the dark about the treatment received. This is considered important in case of subjective symptoms like pain. According to the principles of EBM, this type of experimental design curtails the possibility of biased cognition and is deemed the most reliable and 'objective' measurement of a treatment's effect. The Institute, 
following the EBM-method, attributed reduced credibility to the individual testimonies of patients and urologists due to considerations of potential cognitive bias. From the perspective of epistemic injustice, one could say that the EBM-method in this case regards both patients and doctors as - to some extent - 'lacking the ability to make reliable assertions' (Hookway, 2010, p. 152). Not deliberately, but we can at least conclude that patients and urologists were 'marginalized' as credible epistemic agents with the EBM-method (Carel \& Kidd, 2014, p. 530).

The Institute explained that 'the fact that individual patients claim to benefit from the treatment is not sufficient to conclude that the treatment complies with [reimbursement criteria]', and that the Institute 'does not issue statements about and on behalf of individual patients' (email\#16122013). Rather, the Institute must consider the limited public funds available and ensure that our citizens, who pay the premiums, are paying for something that is worthwhile -involving not an individual person, but the public at large. According to one of the directors, using EBM's quantitative methods allowed the Institute to make such calculations.

"the use of non-personal statistical data on a group level makes it possible to weigh up the personal interests of one group of patients against those of another group of patients. Every euro that you spend on bladder instillation liquids is no longer available for spending on care for elderly persons suffering from dementia, to name just one..." (notes\#25062015)

While the Institute may have recognized patients and doctors 'as possessors and transmitters of knowledge', they did not recognize individual patients' and doctors' testimonies as a format of information that was 'useful in the current context' of making public reimbursement decisions (Hookway, 2010, p. 158).

Is this a form of testimonial injustice? According to Hookway, meaningful epistemic participation requires 'that we be charitable in our understandings, willing to explore the possible uses of the contributions that have been made' (Hookway, 2010, p. 161). Epistemic injustice lurks when the 'hermeneutical offerings' of a patient (or a doctor) is not judged as 'epistemically authoritative' in itself or even treated as an 'epistemically distinctive form of knowledge' (Carel \& Kidd, 2014, p. 533). But what can reasonably be expected of policymakers? It would be impossible to recognize all individual testimonies in a public decision-making context. Effective ways to include patients' knowledge in public decision-making are still being developed and researched (e.g. Lavis, Permanand, Oxman, Lewin, \& Fretheim, 2009), also by the Institute (Kalf, Makady, Ten Ham, Meijboom, \& Goettsch, 2018; Makady et al., 2017).

The EBM-method seems to provide the Institute with a much-needed 'logic of knowledge consumption' (Knorr Cetina, 2007, p. 368) that allows the Institute to make aggregate decisions on population level, that are justifiable as impartial and 'objective' in the public arena. The more pressing question in this case is whether patients (as a group of knowers) had enough epistemic resources to make themselves 'intelligible' within this EBM-formatted public space. Next, we explore 
the question of hermeneutical injustice: did patients have a fair share of our 'collective interpretative resources' at their disposal to manifest their experiences within the EBM-method (Fricker, 2007, p. 1)?

\section{Hermeneutical (in)justice}

The EBM-method used by the Institute provided two opportunities for IC-patients to make their experiences intelligible in the decision-making process: 1) new positive quantitative research 2) new positive quantitative subgroup research. First, the Institute stressed that 'if new positive studies are published, [the Institute] can revise their standpoint' (email\#16122013). This meant that a positive reimbursement decision was possible if patients could present studies that captured their positive experiences with this treatment in a study with the methodological design of an RCT. Second, the Institute insisted throughout the lawsuit that 'when [subpopulation] research becomes available, a new assessment could take place that may result in a different opinion' (statement of defense). The Institute would consider reimbursement for specific groups of IC-patients with proven benefit from this treatment provided that new subgroup studies were published that showed such a positive effect. The Institute recognized that 'based on the literature [...], there is a vague idea that a subgroup exists' (email\#14012014). (Literature suggests, for example, that this treatment works especially for patients with Hunner's lesions in the bladder.) This meant that a positive reimbursement decision was possible if IC-patients could make their experiences intelligible in new quantitative subgroup research (RCT). During legal proceedings, the ICP presented several scientific articles and conference abstracts of promising studies, none of these studies, however, had the experimental design of an RCT. Furthermore, no subgroup studies were available. Hence, the Institute did not change its standpoint.

In her final verdict, the judge considered that patients were not wronged in the Institute's decision-making process. She argued that the Institute had correctly followed the EBM-procedure and had also given patients the opportunity to provide new evidence that the Institute may have missed. The final verdict read: 'it has been established that the information that the ICP could have supplied - and which was supplied during these proceedings - did not lead to a different assessment' (ECLI:NL:RBAMS:2014:772, emphasis added). With that verdict the court upheld the Institute's standpoint not to reimburse bladder instillations with chondroitin sulphate or hyaluronic acid.

According to the patients (ICP) and urologists (NVU), however, translating the positive experiences of clinicians and patients with this treatment into the type of experimental evidence required for reimbursement was not possible. Urologists explained in a letter to the Institute that IC is a spectrum of urological symptoms with different (often unknown) underlying causes and its sufferers are likely to respond differently to treatment: 
'In practice of state-of-the-art clinics that treat patients with IC, this means it is not possible to produce such hard scientific evidence to show which treatments work and which do not. Large series are impossible due to the diversity of the groups of patients and the way in which IC presents, which means that hard evidence will never be supplied' (letter from the urologists' association NVU).

From the perspective of epistemic injustice: do patients who can only participate in decision-making if their contribution comes in the shape of quantitative, statistical (subgroup) evidence truly get a chance to epistemically participate? According to urologists, the required type of quantitative evidence could not be produced. The above statement shows that urologists considered the opportunity to provide new positive RCT studies methodologically infeasible. Moreover, one of the Institute's scientific advisory members remarked about the feasibility of subgroup research: "who would want to carry out an RCT involving something that doesn't work?" (notes\#30302014). Whether the research community would ever conduct further subgroup research is questionable. IC does not easily attract research attention or funds (in contrast to, for example, breast cancer or HIV/ Aids). This means that IC-patients are often left to the mercy of a few dedicated experts (Shao et al., 2010). This is a group of patients with minimal investigative resources at their disposal to make sense of their experiences. Though the requirement of subgroup analysis may seem hypothetically rigorous from an EBM-perspective, in practice, it is unrealistic.

This means that IC-patients had (and still have) meager research resources to make themselves intelligible within the EBM-method. This is more than a case of 'circumstantial epistemic bad luck' (Fricker, 2007, p. 152). Most clinical research has 'minimal patient input', which often means that studies reflect outcomes that matter to researchers, rather than those that matter to patients (Greenhalgh et al., 2015, p. 2; Wiering, de Boer, \& Delnoij, 2017). Consequently, patients participate only marginally 'in the collective practice of interpretation and understanding of their medical situation' (Carel \& Kidd, 2014, p. 532) and they have few entry points or resources to generate the required research. It was with this considerable 'hermeneutical disadvantage' (Fricker, 2007, pp. 151-152) that IC-patients participated in the EBM-formatted decision-making process.

\section{QUESTIONS REGARDING EPISTEMIC INJUSTICE IN PERFORMANCE MEASUREMENT}

Since the introduction of the 2006 Health Insurance Act, private insurance companies play a prominent role in the Dutch healthcare system of managed competition. Insurers and hospitals are free to negotiate prices and selectively contract a range of hospital care products (Van de Ven \& Schut, 2008). Insurers can steer their customers away from hospitals that do not reduce their prices or improve quality. Selective contracting is thought to stimulate both quality and efficiency (Bijlsma, Boone, \& Zwart, 2009). 
For selective contracting, insurance companies need comparative information about the performance of healthcare providers in order to negotiate price and quality (Delnoij, Rademakers, \& Groenewegen, 2010; Enthoven \& Van de Ven, 2007). As of April 1 $1^{\text {st }}$ 2014, the National Health Care Institute is lawfully tasked to provide understandable, comparable and trustworthy information about the quality of care. As part of its official tasks, the Institute promotes the development of quality measures (e.g. quality standards, clinical guidelines, performance indicators, etc.) and stimulates the implementation of quality metrics.

Our second case is about the Institute's mediation in a dispute between doctors, hospitals and insurers about the use of performance indicators in decision-making on the centralization of emergency care in the Netherlands (Moes et al., 2018). This case offers the opportunity to analyze questions regarding epistemic injustice that arise in performance measurement.

\section{The Case}

In 2013, the Association of Dutch Health Insurers (Zorgverzekeraars Nederland, hereafter 'ZN') published a set of outcome, process and structure indicators to measure the quality of hospitals' care for multi-trauma, acute myocardial infarction, cerebrovascular accident, (ruptured) abdominal aortic aneurysm, natal care and hip fracture. The indicators were designed to enable 'insurers to shape their selective care purchasing' (Zorgverzekeraars Nederland, 2013, p. 5). By the end of 2013, insurers had started to use ZN's indicators to compare hospitals in the various regions and started negotiating which centers would (preferably) provide services for emergency cardiology, emergency neurology, traumatology, urgent vascular surgical care, and obstetrics. The idea was to centralize these services in specialized centers where indicators suggested that this was desirable (Zorgverzekeraars Nederland, 2013).

Insurers' plans had far-reaching consequences for individual medical centers and caused much debate amongst emergency care professionals. When negotiations between insurers and hospitals started, a disappointed hospital director stated: "Emergency room closed, no more obstetrics, nor stroke care, no balloon angioplasty, and even broken hip operations will have to be done elsewhere" (Joost Visser, 2014a). Professional media reported: 'In Rotterdam most complex emergency care will move to Erasmus Medical Center. Soon four times as many people will be going there with a stroke. Five hospitals will lose stroke care' (Van Aartsen, 2014b). Medical specialists feared a 'cascading effect': if a hospital loses its contract for the treatment of, for example, myocardial infarction, general cardiology in that hospital could deteriorate or even vanish completely (Joost Visser, 2014b). Professional media reported that hospitals' positions in the debate about insurers' plans were determined by interests: 'hospitals that lose care tasks often object, while the winners see only the advantages' of insurers' plans (Bart Kiers, 2014b).

Doctors, hospitals and professional organizations working in the field of Dutch emergency care contested, furthermore, the accuracy of the indicators and the appropriateness of insurers' use of them. Field parties were concerned about insurers' lack of medical-technical and practical knowledge about emergency care (Bart Kiers, 2014b; Van Aartsen, 2014a). The Dutch Hospitals' 
Association (NVZ) felt that insurers' plans were 'not properly substantiated' and claimed that such indicators 'must be based on scientific research and have the support of medical specialists' (Bart Kiers, 2014b). Apart from battling about resources, this was a knowledge dispute. It was a fight about whose job it was to determine the parameters for performance.

Next, we analyze the indicator-debate from the perspective of epistemic injustice. In the formulation and interpretation of the quality indicators for emergency care: were different stakeholders duly recognized in their capacity as knowers of emergency care? And did the different stakeholders have the hermeneutical resources to participate meaningfully in the process of drawing up (and putting to use) these indicators?

\section{Hermeneutical (in)justice}

Although representatives of the Dutch professional associations were not directly involved in formulating ZN's quality indicators, ZN did consult medical professionals' knowledge about good quality emergency care as represented in professional and academic literature. $\mathrm{ZN}$ based its indicators on a vast number of scientific publications, including RCTs, systematic reviews and cohort studies, as well as Dutch, European and international guidelines (Zorgverzekeraars Nederland, 2013). As such, medical professionals' 'hermeneutical offerings' were actually judged as 'epistemically authoritative' in the formulation of ZN's performance indicators (Carel \& Kidd, 2014 , p. 533). Medical professionals, as a group of knowers, were part of the collective practice of interpreting and understanding the quality of emergency care and determining parameters for performance. No hermeneutical injustice occurred, as it was not 'a gap in collective interpretive resources' that put medical specialists, hospitals and other professional organizations at an 'unfair disadvantage' in making sense of the quality of Dutch emergency services (Fricker, 2007, p. 1). Any potential epistemic injustice lay elsewhere.

\section{Testimonial (in)justice}

Most parties considered the insurers' initiative a top-down exercise. In professional media, the spokesperson for hospitals in the Northern region claimed, that

"no advance consultation took place with hospitals, the platform for acute care, GPs and patients' organizations. We were confronted with a fait accompli. Lists with green and red ticks. And would we formalize the plans within two weeks. You can't simply impose a new model from your ivory tower" (Bart Kiers, 2014a).

While insurers regarded the negotiations as a collaborative process - "discussions are going well, people are involved" - some hospitals felt that insurers' plans had been "set in stone" and that insurers were "not interested in the vision of the NVZ or any alternative plans the hospitals might have" (Joost Visser, 2014a). Hospitals felt that their knowledge about the quality of emergency services had not been heard or taken seriously by insurers. 
According to Hookway, epistemically meaningful participation 'is not just a matter of exchanging information; it involves asking questions, floating ideas, considering alternative possibilities and so on' (Hookway, 2010, p. 156). The arguments exchanged in professional media as presented above show that both the professional community and local healthcare providers felt that they did not truly participate in deliberations.

Quality indicators, such as the ones used by ZN, follow the logic that clinical science can determine 'what works' and parameters based on these scientific findings can form an impartial and objective standard for provider behavior. Academic work has shown that quantified clinical knowledge is 'easily transformed' into a type of 'business knowledge of pathways and protocols' such as performance indicators (Mykhalovskiy \& Weir, 2004, p. 1061). For non-medical parties who have no access to the primary care processes - such as, in this case, insurers - this aggregate knowledge provides the easiest way to gain insights into 'what works' in clinical practice. The use of such quantified parameters is typically presumed to hold to ideals of impartiality, objectivity and justice in measuring and comparing the quality of different healthcare services.

However, the use of such standardized protocols has also been shown to challenge the clinical autonomy and professional judgement of local healthcare providers (Greenhalgh, 2002; Knaapen, 2014; Lambert, 2006; Timmermans \& Berg, 2003; Timmermans \& Kolker, 2004). Performance measures, in other words, undermine the 'medical professional's privileged authority to evaluate [his/her] own work' (Knaapen, 2014, p. 829). It is 'testimonial justice' that is at stake when the testimonies of local professionals threaten to be pushed aside by the facts and figures of external parties who attribute more credibility to parameters formulated by the medical profession at large than to the embodied, contextual knowledge of local providers. Next, we study the mediation work of the National Health Care Institute and show how their work reinstalled local stakeholders as epistemic agents in the deliberations.

The Institute became involved in the debate based on its role as authoritative public knowledge institution working on providing reliable information about the quality of care. After several rounds of consultations with professional organizations, the Institute problematized two things. First, the scope of the indicator set. Field parties considered the scope of ZN's indicators too narrow and wanted to broaden it to the full spectrum of medical emergencies (not restricted to only six indications). They also considered it important to take into account the patients' trajectory from emergency call to aftercare (not just care that patients received in the ER). Therefore the Institute facilitated the development of a comprehensive 'Emergency Care Chain Quality Framework' (encompassing the full spectrum of emergency services and the entire patient trajectory) designed by all parties involved: professional organizations of patients, general practitioners, ambulance staff, primary healthcare, hospitals, academic medical centers, medical specialists, emergency doctors and health insurers. As such, the Institute explicitly reinstated all the different stakeholders as relevant epistemic agents in deliberating and making sense of the quality and efficiency of Dutch emergency services and how they should be organized. 
Second, the Institute installed a group of thirteen experts representative of the professional emergency care community to provide a formal assessment of the original set of performance indicators. While the committee found that there was sufficient evidence supporting most of the performance indicators and that they were "not unreasonable", they also stressed that the standardized knowledge of indicators alone was not enough to make sense of the quality of emergency services. A committee member explained that the quality of treatment for a heart attack, for example,

"...starts with a patient raising the alarm in good time, after which the GP takes a look, who then refers the patient to hospital in good time, and all of that finally determines the outcome of a myocardial infarction. If you judge a hospital based on, for instance, survival rates for myocardial infarction, well then you would also have to take into account the part of the chain before the hospital... If people call in the GP too late, or the GP doesn't do his job properly, you will be running the risk that indicators will work against hospitals. You have to realize this, if you are going to make use of this sort of indicator..."

While ZN's outcome indicators made sense in themselves, the quality of treatment for a heart attack depends on the entire trajectory from incident to aftercare. The committee stressed that the indicators did not suffice to make sense of the performance of individual hospitals. With "evidencebased medicine as the foundation of the quality movement", the committee's chairperson explained, the focus had moved to "standardized protocol quality." But in applying such standards "local data are important" and collaboration in the region is necessary to exploit sources of local, tacit and contextual knowledge. As such, the chairperson problematized insurers' use of standardized knowledge:

"This was just one party... and a party that was at a considerable distance... and because [the insurers] are at such distance, their only weapon is population data from the evidence-based medicine world... which is standardized... If someone has no hands-on experience and has never actually been to an emergency room... then these are the only available data for him to use. But, then he forgets the knowledge sources that are somewhat harder for him to exploit, but that are very important too..."

Ultimately, the committee emphasized that the performance indicators were to be used by professionals to jointly discuss how best to organize emergency services in the particular regions (Zorginstituut Nederland, 2015, p. 15). The expert committee, by stressing the importance of contextualizing the standardized performance measures with local knowledge, reinstalled local emergency care professionals as relevant epistemic agents in the process of making sense of the quality of regional emergency care services and putting the quality indicators to use. 
The fact that this type 'repair work' had to be taken up by the Institute is a reminder that testimonial injustice lurks when non-medical parties - who have no access to primary care processes - resort to the use of quantified clinical parameters. The quantitative approach of performance indicators suggests the possibility of separating 'expertise from expert, and knowledge from knower' (Tanenbaum, 1995, p. 102). This has given actors without direct experience of the primary care process the opportunity to 'regulate the field of healthcare and hold it accountable using [...] parameters formulated by the professions' (Timmermans \& Berg, 2003, p. 20). Quantified parameters are typically presumed to warrant impartiality, objectivity and justice in measuring and comparing the quality of health services. Chances are, however, that when high levels of credibility are attached to such quantified parameters, the credibility of embodied and non-standardized knowledge of local health professionals is unduly marginalized.

\section{CONCLUDING REMARKS}

In this paper we studied the usefulness and limits of Fricker's concept of epistemic injustice in the context of public decision-making. In contemporary healthcare policies the logic of EBM is central to how 'knowing is done' (de Laet, 2012). Evidence-based decision-making is typically proposed as a way of addressing a growing demand to explicitly justify decisions in the public arena (Dobrow et al., 2004; Parkhurst, 2017). Policymakers' use of scientific knowledge - 'evidence' in particular - is presumed to pertain to ideals of impartiality, objectivity and justice in decision-making and to guard policymakers against the suspicion of arbitrariness or bias (Porter, 1994). However, because EBMinspired policy practices 'privilege' certain types of evidence, certain ways of presenting knowledge and certain styles of articulating testimonies, they can also be 'liable to generate epistemic injustice' (Carel \& Kidd, 2014, pp. 530-531).

In this paper we analyzed two cases of EBM-inspired policy practices in Dutch social health insurance and studied how knowledge work in policymaking is intertwined with questions regarding epistemic justice. Our analysis of the first case illustrated that the embodied knowledge of patients and urologists was (unintentionally) marginalized as credible and useful information in the Institute's EBM-based reimbursement decision. In particular, patient testimonies are still seen as subjective, 'anecdotal', limited to the 'private realm', 'context-dependent', 'as numerous as patients', and, therefore, difficult to use in public decision-making settings (Carel \& Kidd, 2014, p. 535). However, can policymakers reasonably be expected to hear all patient testimonies in a public setting where different interests are at stake? EBM's principles provide policymakers with a much needed 'logic of knowledge consumption' (Knorr Cetina, 2007) to make aggregate decisions on population level that are explicitly justifiable as 'objective', 'impartial' and 'just' in the public arena. These quantified procedures are actually designed to facilitate the conscious exercise of counteracting bias and to prevent discriminatory outcome in policymaking. But, while formal institutions and their procedures 
for policymaking may be considered just, impartial and objective, this does not ensure that epistemic injustice is not occurring. Our analysis showed that patients had meager resources to produce the 'hard evidence' with which they could make themselves intelligible within the EBM-formatted public decision-making. If this type of hermeneutical marginalization is not recognized in policymaking, decisions may be regarded as upholding ideals of procedural fairness, but may still be epistemically unjust and potentially lead 'to misrepresentations in collective preferences' (Bernal, 2018, p. 23; Moes et al., 2017).

The second case illustrated that questions regarding meaningful epistemic participation are equally relevant in making decisions in the context of the healthcare market. Performance indicators follow the same logic as EBM: clinical science can determine 'what works' and parameters based on these quantified findings can form an objective standard for provider behavior (Eddy, 2005; Tanenbaum, 2012). The use of performance indicators based on quantified clinical knowledge has given non-medical parties the possibility to act independently in a field where - originally - they had no epistemic authority (see Tanenbaum, 2012). The second case illustrated that when the protocolled and quantified medical knowledge of performance metrics aligns to match the logic of the insurers' market, the embodied, contextual knowledge of local professionals is potentially silenced. In that case it is questionable whether indicators still serve to promote the quality of care.

Application of the concept of epistemic injustice to policy practices shows that when groups of knowers (whether patients or local professionals) are not duly recognized as epistemic agents, they subsequently lack the social power to influence priority-setting practices in which their interests are at stake. These groups are not merely frustrated in their epistemic capacity to be heard and make themselves understood, they are - as a result - potentially deprived of a fair share in our collective financial and medical resources. If we fail to recognize inequalities in credibility and intelligibility between diverse groups of knowers, there is a chance that these latent epistemic inequalities are being reproduced in our system of health insurance and our ways of distributing healthcare provisions.

It is important to stress that both international literature and the National Health Care Institute have long moved beyond a naive faith in the ability of EBM to straightforwardly guide complex priority-setting decisions (Russell, Greenhalgh, Burnett, \& Montgomery, 2011). Aware of the shortfalls of EBM's strict hierarchy, the Institute now makes use of, for example, GRADE (see e.g. Guyatt et al., 2008), an EBM-method that allows more flexibility within the original hierarchy. The Institute is also exploring how to use 'real-world data' (Makady et al., 2017) and patient experiences from social media in health technology assessments (Kalf et al., 2018). This 'repair and maintenance work' shows that policymakers continue to search for ways to meaningfully combine patients' and professionals' embodied, qualitative knowledge with aggregate, quantitative knowledge in policymaking. Epistemic injustice, however, often precedes such repair work. It is, therefore, important that our priority-setting practices are continuously evaluated from the perspective of epistemic injustice. 
The concept of epistemic injustice, however, misses some key processes involved understanding how 'knowing gets done' in public policy processes. This has much to do with the infrastructure on which policymaking - in this case priority-setting - draws: current (international) procedures, conventions, regulations, stakeholders, etc. Fricker focusses on the role of the virtuous and unprejudiced listener, emphasizing the interrelational aspects of epistemic injustice. However, in the context of public decision-making, epistemic injustice typically does not originate between agents, but in the knowledge infrastructure from which agents draw. It is often through the work of policymakers that deeper, structural epistemic inequalities are made visible, and it is important not to reproduce these inequalities in priority-setting. But, where were these injustices produced in the first place (Epstein, 2007)? Who is actually responsible for the widespread lack of credibility attributed to patient testimonies? In order to apply the concept of epistemic injustice adequately in the public context, we need to explore much more thoroughly the question of what 'epistemic justice' as a virtue requires of social institutions (Anderson, 2012). What does epistemic responsibility look like in a collective context (cf. Isaacs, 2011)? If we want to further develop and use the concept of epistemic injustice to investigate policy practices, these questions require our explicit attention. 


\section{REFERENCES}

Alcoff, L. M. (2010). Epistemic Identities. Episteme, 7(2), 128-137.

Anderson, E. (2012). Epistemic Justice as a Virtue of Social Institutions. Social Epistemology, 26(2), $163-173$.

Armstrong, D. (2002). Clinical autonomy, individual and collective: the problem of changing doctors' behaviour. Social science \& medicine (1982), 55(10), 1771-1777.

Bernal, A. (2018). Epistemic Overload as Epistemic Injustice. Blacksburg, Virginia.

Bijlsma, M., Boone, J., \& Zwart, G. (2009). Selective Contracting and Foreclosure in Health Care Markets. CentER Discussion Paper Series No. 2009-89, TILEC Discussion Paper No. 2009-045.

Carel, H., \& Kidd, I. (2014). Epistemic injustice in healthcare: a philosophial analysis. Medicine, Health Care and Philosophy, 17(4), 529-540.

Coady, D. (2010). Two Concepts of Epistemic Injustice. Episteme, 7(2), 101-113.

College voor Zorgverzekeringen. (2007). Beoordeling stand van de wetenschap en praktijk. Diemen: College voor Zorgverzekeringen (CVZ).

College voor Zorgverzekeringen. (2013a). Achtergrondrapportage beoordeling stand van de wetenschap en praktijk. Blaasvloeistoffen met chondroïtinesulfaat en/of hyaluronzuur (Vol. 2013084487). Diemen: College voor Zorgverzekeringen (CVZ).

de Laet, M. (2012). Anthropology as Social Epistemology? Social Epistemology, 26(3-4), 419-433.

Deaton, A., \& Cartwright, N. (2018). Understanding and misunderstanding randomized controlled trials. Social science \& medicine, 210, 2-21.

Delnoij, D., Rademakers, J., \& Groenewegen, P. (2010). The Dutch Consumer Quality Index: an example of stakeholder involvement in indicator development. BMC Health Services Research, 10(88).

Dieleman, S. (2015). Epistemic Justice and Democratic Legitimacy. Hypatia, 30(4), 794-810.

Dobrow, M., Goel, V., \& Upshur, R. (2004). Evidence-based health policy: context and utilisation. Social science \& medicine, 58(1), 207-217.

Eddy, D. (2005). Evidence-Based Medicine: A Unified Approach. Health Affairs, 24, 9.

Enthoven, A., \& Van de Ven, W. (2007). Going Dutch - Managed-competition health insurance in the Netherlands. The New England Journal of Medicine(357), 2421-2423.

Epstein, S. (2007). Inclusion: the Politics of Difference in Medical Research: University of Chicago Press.

Evidence-Based Medicine Working Group. (1992). Evidence-based medicine. A new approach to teaching the practice of medicine. JAMA, 268(17), 2420-2425.

Felt, U., \& Fochler, M. (2010). Machineries for Making Publics: Inscribing and De-scribing Publics in Public Engagement. Minerva, 48(3), 219-238.

Fricker, M. (2007). Epistemic injustice: power and the ethics of knowing. Oxford: Oxford University Press.

Ghinea, N., Lipworth, W., \& Kerridge, I. (2015). Evidence, regulation and rational prescribing: the case of gabapentin for neuropathic pain. Journal of Evaluation in Clinical Practice, 21(1), 28-33.

Green, J., \& Thorogood, N. (2004). Qualitative Methods for Health Research. London: SAGE Publication.

Greenhalgh, T. (2002). Intuition and evidence - uneasy bedfellows? British Journal of General Practice, 52, $395-400$. 
Greenhalgh, T., \& Russell, J. (2009). Evidence-Based Policymaking: A Critique. Perspectives in Biology and Medicine, 52(2), 304-318.

Greenhalgh, T., Snow, R., Ryan, S., Rees, S., \& Salisbury, H. (2015). Six 'biases' against patients and carers in evidence-based medicine. BMC Medicine, 13, 200.

Guyatt, G., Oxman, A., Vist, G., Kunz, R., Falck-Ytter, Y., Alonso-Coello, P., \& Schünemann, H. (2008). GRADE: an emerging consensus on rating quality of evidence and strength of recommendations. BMJ, 336(7650), 924-926.

Guyatt, G., Sackett, D., Sinclair, J., Hayward, R., Cook, D., \& Cook, R. (1995). Users' Guides to the Medical Literature: IX. A Method for Grading Health Care Recommendations. JAMA, 274(22), 1800-1804.

Helderman, J., De Kruijf, J., Verheij, J., \& Van Thiel, S. (2014). Dike-Reeve of the health care polder. A politicalsociological analysis of the realisation of the National Health Care Institute against a backdrop of a changing policy agenda and changing political-administrative and societal relations. Diemen: Zorginstituut Nederland.

Hookway, C. (2010). Some Varieties of Epistemic Injustice: Reflections on Fricker. Episteme, 7(2), 151-163.

Irwin, A. (2006). The Politics of Talk: Coming to Terms with the 'New' Scientific Governance. Social Studies of Science, 36(2), 299-320.

Isaacs, T. (2011). Moral responsibility in collective contexts. New York: Oxford University Press.

Kalf, R., Makady, A., Ten Ham, R., Meijboom, K., \& Goettsch, W. (2018). Use of Social Media in the Assessment of Relative Effectiveness: Explorative Review With Examples From Oncology. JMIR cancer, 4(1).

Kidd, I., \& Carel, H. (2017). Epistemic Injustice and Illness. Journal of Applied Philosophy, 34(2), 172-190.

Kiers, B. (2014a, 13 March 2014). Ziekenhuizen maken gehakt van spoedplan. Zorgvisie

Kiers, B. (2014b, 16 July 2014). ACM haalt streep door concentratie spoedzorg. Zorgvisie

Knaapen, L. (2014). Evidence-Based Medicine or Cookbook Medicine? Addressing Concerns over the Standardization of Care. SOC4 Sociology Compass, 8(6), 823-836.

Knorr Cetina, K. (2007). Culture in global knowledge societies: knowledge cultures and epistemic cultures. Interdisciplinary Science Reviews, 32(4), 361-375.

Lambert, H. (2006). Accounting for EBM: Notions of evidence in medicine. Social science \& medicine, 62(11), 2633-2645.

Langton, R. (2010). Epistemic Injustice: Power and the Ethics of Knowing. By MIRANDA FRICKER. Hypatia, 25(2), 459-464.

Lavis, J., Permanand, G., Oxman, A., Lewin, S., \& Fretheim, A. (2009). SUPPORT Tools for evidence-informed health Policymaking (STP). 13. Preparing and using policy briefs to support evidence-informed policymaking. Health Research Policy and Systems, 7(1).

Maitra, I. (2010). The Nature of Epistemic Injustice. Philosophical Books, 51(4), 195-211.

Makady, A., ten Ham, R., de Boer, A., Hillege, H., Klungel, O., \& Goettsch, W. (2017). Policies for Use of RealWorld Data in Health Technology Assessment (HTA): A Comparative Study of Six HTA Agencies. Value in Health, 20(4), 520-532.

Michael, M. (2012). "What Are We Busy Doing?": Engaging the Idiot. Science, Technology, \& Human Values, 37(5), 528-554.

Moes, F., Houwaart, E., Delnoij, D., \& Horstman, K. (2017). Contested evidence: a Dutch reimbursement decision taken to court Health economics, policy, and law, 12(3), 325-344.

Moes, F., Houwaart, E., Delnoij, D., \& Horstman, K. (2018). "Strangers in the ER": Quality indicators and third party interference in Dutch emergency care. Journal of Evaluation in Clinical Practice, 1v8. 
Mykhalovskiy, E., \& Weir, L. (2004). The problem of evidence-based medicine: directions for social science. Social science \& medicine, 59(5), 1059-1069.

Parkhurst, J. (2017). The Politics of Evidence: From evidence-based policy to the good governance of evidence. New York: Routledge.

Peeters, M., Delnoij, D., \& Friele, R. (2014). Stronger, but not (yet) an equal. The use of quality improvement instruments and strategies by patient organisations in the Netherlands. Social science \& medicine, 115 , 56-63.

Porter, T. (1994). Making things quantitative. SCIENCE IN CONTEXT, 7, 389-407.

Russell, J., Greenhalgh, T., Burnett, A., \& Montgomery, J. (2011). "No decisions about us without us"? Individual healthcare rationing in a fiscal ice age. $B M J, 342$ (jun13 1), d3279.

Sackett, D., Rosenberg, W., Muir Gray, J., Haynes, R., \& Richardson, W. (1996). Evidence based medicine: what it is and what it isn't. BMJ, 312(7023), 71-72.

Shao, Y., Shen, Z.-J., Rui, W.-B., \& Zhou, W.-L. (2010). Intravesical Instillation of Hyaluronic Acid Prolonged the Effect of Bladder Hydrodistention in Patients With Severe Interstitial Cystitis. Urology, 75(3), 547-550.

Sherman, B. (2016). There's No (Testimonial) Justice: Why Pursuit of a Virtue is Not the Solution to Epistemic Injustice. Social Epistemology, 30(3), 229-250.

Staal, P., Heymans, J., Ligtenberg, G., Derksen, J., \& Couwenbergh, B. (2015). Beoordeling stand van de wetenschap en praktijk. Diemen: Zorginstituut Nederland.

Tanenbaum, S. (1995). Getting there from here: evidentiary quandaries of the US outcomes movement. Journal of Evaluation in Clinical Practice, 1(2), 97-103.

Tanenbaum, S. (2012). Improving the quality of medical care: the normativity of evidence-based performance standards. Theoretical medicine and bioethics, 33(4), 263-277.

Timmermans, S., \& Berg, M. (2003). The Gold Standard: the challenge of evidence-based medicine and standardization in health care. Philadelphia, PA.: Temple University Press.

Timmermans, S., \& Kolker, E. (2004). Evidence-Based Medicine and the Reconfiguration of Medical Knowledge. Journal of Health and Social Behavior, 45, 177-193.

Van Aartsen, C. (2014a, 5 February 2014). NVZ clasht met zorgverzekeraars over spoedzorg. Zorgvisie.

Van Aartsen, C. (2014b, 31 January 2014). Vijf Rotterdamse ziekenhuizen verliezen beroerte-zorg. Zorgvisie.

Van de Ven, W., \& Schut, F. (2008). Universal mandatory health insurance in the Netherlands: A model for the United States? Health Affairs, 27(3), 771-781.

Van de Ven, W., \& Schut, F. (2009). Managed competition in the Netherlands: Still work-in-progress. Health Economics, 18(3), 253-255.

Visser, J. (2014a, 12 February 2014). Concentratie spoedzorg ligt zwaar op de maag. Medisch Contact.

Visser, J. (2014b, 30 July 2014). Concentratie van spoedzorg is hooguit afgeremd. Medisch Contact.

Wiering, B. M., de Boer, D., \& Delnoij, D. (2017). Patient involvement in the development of patient-reported outcome measures: A scoping review. Health Expectations, 20(1), 11-23.

Zorginstituut Nederland. (2015). Spoed Moet Goed: Indicatoren en Normen voor Zes Spoedindicaties. Diemen: Zorginstituut Nederland.

Zorgverzekeraars Nederland. (2013). Kwaliteitsvisie Spoedeisende Zorg. Hoofdrapport. Zeist: Zorgverzekeraars Nederland. 



\section{Conclusions}

\section{Experts and Idiots}




\section{GINO, THE IDIOTIC CATALYST}

“You'll see, Gino is pretty unsociable. He doesn't talk. He's really withdrawn. I don't know what he'll find to tell you but I'm really glad he eventually agreed to see you. I really thought he was going to refuse", Léon tells sociologists Callon and Rabeharisoa (Callon \& Rabeharisoa, 2004, p. 2). The sociologists are on La Reunion Island (France) to interview Léon and his brother Gino about the Limb-Girdle Muscular Dystrophy (LGMD) that affects both brothers. LGMD is a hereditary genetic disorder that typically appears in adolescence and worsens over time. While some patients have mild symptoms such as an unusual walking gait (swaying or walking on the balls of the feet), others are condemned to wheelchairs at an early age. Gino portrays hardly any clinical signs of LGMD; he is what some MD-patients might call 'as fit as a fiddle' (Callon \& Rabeharisoa, 2004, p. 2). In their paper 'Gino's lesson on humanity', Callon and Rabeharisoa narrate their encounter with Gino:

'It was difficult to get anything out of him other than a few mumbled and sometimes inaudible words. Most of the time his brother and wife answered for him... on three occasions only did he join in the conversation' (Callon \& Rabeharisoa, 2004, p. 3).

The first time Gino entered into the conversation was with regard to his refusal of medicine. LGMD appeared when Gino was around 30. The sociologists ask Gino if he went to see a doctor at that time. Gino's response: “I didn't go to see a doctor. In any case, I knew from the family what I had and knew there was no cure. There was no point in going to see a doctor. When I was tired at work I rested, that's all. There's nothing else to do" (Callon \& Rabeharisoa, 2004, p. 3). The second time Gino entered into the conversation was with regard to his refusal to participate in the La Reunion Island Muscular-dystrophy Association (ARM). Sociologists: "Do you participate in the ARM's activities?" Gino: "No, I don't participate in the ARM. When I was working I didn't have the time. Now I prefer going to a football match. I like football" (Callon \& Rabeharisoa, 2004, p. 4). The third time Gino joined the interview was with regard to his refusal to 'know' about genetic inheritance. Gino's brother and wife inform him about genetic testing and how his children and grandchildren are potential carriers of the gene. The sociologists tentatively ask Gino: "Doesn't this make you a bit worried?" to which Gino responds after hesitating a while: "It worries me a little but... [he hesitates again, then smiles] l'd rather not know" (Callon \& Rabeharisoa, 2004, p. 5).

Gino's behavior in the interview leaves the sociologists with what they call 'disturbing questions' (Callon \& Rabeharisoa, 2004, p. 5). How should Gino's responses - or rather the lack of responses - be interpreted?

'What is the root of these three refusals? How can they be explained? Should Léon's interpretation be accepted: "You'll see, he's pretty unsociable"? Should Gino be seen as slightly backward or simple-minded, or even simply amoral when he shows no concern for the possible misfortune of his grandchildren? Should we look for mitigating 
circumstances and search in his childhood or influences in his life for a plausible cause for this strange attitude? Should we refer to patients' classical denial of their own disease?' (Callon \& Rabeharisoa, 2004, p. 5)

The sociologists felt that it would be too easy to deal with this uncomfortable encounter by writing Gino off as a 'slightly backward or simple-minded, or even simply amoral' person. They analyze Gino's refusals - not to consult a doctor, not to join the ARM, and not to get his children genetically tested - as a refusal to play a part that is forced onto him, namely 'that of a human being who is ill, who knows that he is ill and what disease he has, and who has to discuss it publicly' (Callon \& Rabeharisoa, 2004, p. 11). They see Gino's behavior as a rejection of a particular form of agency proposed to him by a network of geneticists, the ARM, doctors, ethicists and sociologists; a type of agency that is not his own and to which he is opposed.

Since Michel Fardeau in the 1980s localized and identified the genes responsible for LGMD, the disease has become a public reality that is genetically visible and clinically describable. Since then the disease 'circulates' in professional networks including doctors, physiotherapists, nurses, occupational therapists, hospital services, social action services, politicians and patient associations as well as patients' families (Callon \& Rabeharisoa, 2004, p. 9). As LGMD entered the public space as a hereditary genetic disorder, it brought with it all kinds of rational and moral choices: to follow the doctors' advice or not, to be treated by a physiotherapist or not, deciding to abort or not, having children undergo a test or not, participating in activities organized by the patients' association or not, et cetera (Callon \& Rabeharisoa, 2004, p. 13). There is no public space that does not carry with it a particular rationality and normativity. Hereditary genetics imposes a category of moral action and those who have to make choices in this regard are expected to deliberate on these choices and assume full responsibility for them (Callon \& Rabeharisoa, 2004, p. 19). With the coming into being of professional medico-social networks for LGMD, patients are rationally and morally obliged to explain why they would remain outside of these networks; why they would refuse genetic testing.

Yet, Gino opposes the reconfiguration of his identity that is forced upon him by the upsurge of genetics and the development of a strong medico-social network for LGMD. Gino chooses to remain outside this network and refuses to engage with its specific rationality and morality by drawing on an entirely different array of reasons and morals: he sees no point in going to a doctor; he dislikes banquets and the trips organized by the ARM, instead he likes football and with regard to hereditary genetics he smilingly admits "I'd rather not know". Gino seems to know that accepting genetics 'means sliding into a public space where certain moral questions have to be discussed' (Callon \& Rabeharisoa, 2004, p. 19); that 'to be considered a moral human being, he would have to fit into the mould of this world in which generations are bound to one another by genes that sometimes falter' and enter 'a space of choices where there is a question of tests, marriage, abortion, etc.' (Callon \& Rabeharisoa, 2004, p. 18). Ultimately, Gino's resistance to 'know' genetics and his refusal of medicine and to engage in collective action is not simply the discarding of reason and morality. 
In their remarkable paper, Callon and Rabeharisoa argue that Gino is opting for another form of 'morality and intelligence' (Callon \& Rabeharisoa, 2004).

Gino is a benign nonconformist, an 'idiot' - in Stengers' definition of the word - that 'resists the consensual way in which the situation is presented' (Stengers, 2005, p. 994). The encounter between sociologists and Gino shows that those who refuse to fit the system re-open confusion, uncertainty and ambiguity and can serve as 'a heuristic for interrogating "what we are busy doing"' (Michael, 2012, p. 529). Callon and Rabeharisoa decided not to put Gino's interview aside as the useless contribution of a simple-minded and amoral person. By listening to what Gino had to say (or rather what he refused to say), Gino served as a heuristic for them to reflect on practices of knowing regarding LGMD. Callon and Rabeharisoa show that 'idiotic' events - incomprehensible refusals, embarrassments, miscommunication, et cetera - are perfect opportunities to investigate unexamined practices of knowing.

\section{AN INVITATION TO REFLECT}

Over the past century, everyday social practices - ranging from eating, sleeping and exercising to care and medicine - have been increasingly determined by science and technology. These practices have become characterized by particular ways of knowing that regulate the types of cognitive reasoning and moral argumentation and that can be pursued in that particular area of life. Modern knowledge societies are permeated with such epistemic settings (Knorr Cetina, 2007).

The introductory chapter to this book shows how important it is to reflect on these epistemic practices. Practices of knowing are never neutral. They contain dominant notions of what counts as credible, objective and trustworthy knowledge. As such, they allow for the visibility of certain types of knowledge and styles of reasoning, while others are, at the same time, backgrounded, silenced and made invisible (Murphy, 2006). Callon and Rabeharisoa discovered that their epistemic practices (as well as those of geneticists and ethicists) permitted the articulation of certain arguments and morals, and not others. Had they not used Gino as an 'idiotic catalyst' (Farías, 2017) to examine their own ways of knowing, Gino's voice would have been silenced, backgrounded and made imperceptible in their sociological inquiry.

Fricker coined the term 'epistemic injustice' to refer to this type of wrong done to a person by rejecting their testimony as a credible and intelligible contribution (Fricker, 2007). This book is about epistemic practices in policymaking. For a society that relies heavily on techno-scientific knowledge in making public decisions, it is of vital importance to reflect on the rationality and morality inherent in its practices of knowing, because these practices are central to deciding how to inhabit the public space and how to shape collective futures. Whose knowledge and what types of testimonies are actually counted as objective, credible and trustworthy contributions to policymaking processes? People who are not recognized in their capacity as credible and intelligible epistemic agents in 
policy processes, lack the social power to influence policymaking and are excluded from the deliberative processes that shape public spaces and collective futures.

According to Fricker we can (and should) fight epistemic injustices by guarding against prejudice in assessing and processing the testimonies of others. The work of Callon and Rabeharisoa is a beautiful example of this. This book addresses epistemic practices in social health insurance policy and reflects on the epistemic injustices that potentially circulate in them. This concluding chapter recounts the findings of my ethnographic studies and engages with the question of how we can guard against potential epistemic injustices in the practices of knowing in social health insurance.

In what follows, I first elaborate on epistemic practices in health policymaking and how Evidence-Based Medicine (EBM) has come to play a central role in objectifying and justifying complex decisions in contemporary health care. While EBM has become a wide-spread epistemic ideal in decision-making, at the same time, it has been shown to privilege certain articulations of rationality while silencing others, and - as such - EBM has been criticized for potentially generating epistemic injustices. Second, I report about the main findings from my ethnographic studies at the National Health Care Institute. I explore the role of EBM in the epistemic practices in Dutch social health insurance and how these EBM-formatted epistemic practices can be analyzed from the perspective of epistemic injustice. To conclude, I return to 'the idiot' and explore how idiotic catalysts and dissonant voices are a potential remedy to identify and address the epistemic injustices that might circulate in practices of knowing in social health insurance.

\section{EPISTEMIC PRACTICES IN HEALTH POLICY}

In Western democratic culture, which idealizes the rule of law, it is considered unacceptable if bureaucratic officials rely merely on their personal and potentially prejudiced judgement. Throughout Western democracies we have therefore come to rely heavily on quantified, scientific and technical knowledge to justify actions and to legitimize policy decisions as being 'impartial', 'objective' and 'just' (Ezrahi, 1990; Porter, 1995). As we saw in the introductory chapter to this book, big infrastructural plans are expected to be based on officials' explicit argumentation, drawing from 'objective' measures such as Environmental Impact Reports, sound pollution calculations and traffic prognoses (de Jonge \& Nijland, 2018; de Zeeuw, 2017), just as immigration plans require explicit justification based on 'objective' demographic statistics (Partos, 2014). The use of quantitative, technical and scientific knowledge in democratic decision-making is assumed to prevent policymakers' cognitive biases from being triggered and it aims to avoid discriminatory or prejudiced outcomes in policymaking.

In the field of health care, 'evidence-based' decision-making is typically proposed as a way of addressing the demand to explicitly justify decision-making in public policy (Dobrow, Goel, \& Upshur, 2004; Parkhurst, 2017). EBM was announced as a 'new paradigm' for medicine, promising a shift away from 'intuition, clinical experience and pathophysiologic rationale' towards 'using the 
[scientific] literature more effectively in guiding medical practice' (Evidence-Based Medicine Working Group, 1992, p. 2420). Amid the growing recognition of 'the vagaries of clinical experience' (D. Armstrong, 2002, p. 1772), the idea behind EBM was to teach doctors to critically consume medical literature (Weisz et al., 2007, p. 713), emphasizing the use of experimental evidence. EBM came with a hierarchical classification of medical knowledges according to rigorousness and quality of research methods (with randomized controlled trials (RCT) and systematic reviews forming the apex of the hierarchy) (Guyatt et al., 2008; Guyatt et al., 1995). To support clinicians, to get them to use the best available evidence of 'what works' in medical decision-making, EBM also promoted the use of tools such as clinical guidelines and evidence reports to 'bring the evidence to health care providers' (Knaapen, 2014, p. 824). Originally designed for clinical practice to help physicians improve medical decision-making, EBM - 'the conscientious, explicit, and judicious use of current best evidence' (Sackett, Rosenberg, Muir Gray, Haynes, \& Richardson, 1996) - has become an epistemic ideal for making policy decisions too.

EBM and its focus on quantified, experimental evidence offers procedures for decisionmaking that are thought to counteract cognitively biased, discriminatory or prejudiced outcomes in policymaking and that help in making 'impartial', 'objective' and 'just' health policy decisions. However, some have suggested that contemporary health care practices, that are largely based on the principles of EBM, are nonetheless liable to generate epistemic injustice because they 'privilege certain styles of articulating testimonies, certain forms of evidence, and certain ways of presenting and sharing knowledge' (Carel \& Kidd, 2014, p. 530). EBM's focus on quantitative population-based research potentially undervalues both doctors and patients 'as 'knowers' capable of making judgements outside the confines of epidemiological evidence' (Deaton \& Cartwright, 2018; Ghinea, Lipworth, \& Kerridge, 2015, p. 28; Greenhalgh, 2002; Greenhalgh, Snow, Ryan, Rees, \& Salisbury, 2015). While its founding fathers claim that EBM entails the integration of the best external evidence with individual clinical expertise and patients' choice (Sackett et al., 1996), critics have long been concerned that EBM's focus on epidemiological evidence and a formulaic decision-making model nonetheless undervalues situated clinical expertise of doctors and patients' experiences and preferences (Greenhalgh et al., 2015; Lambert, 2006).

So, while on the one hand EBM is considered a procedural remedy to avoid prejudice or cognitive bias in decision-making, on the other hand it has been shown that EBM contains biases and prejudices of its own. It is for this reason that, in my analyses of the epistemic practices of the National Health Care Institute, I focused on the role of EBM in making and justifying public policy decisions, and how these epistemic practices could be analyzed from the perspective of epistemic injustice. 


\section{DOING EPISTEMIC JUSTICE IN DUTCH SOCIAL HEALTH INSURANCE}

In the Netherlands, EBM has been instrumental - in recent decades - in achieving 'objectivity' and explicit justification of complex policy decisions and, as such, aligning the government and the different field parties whenever difficult decisions had to be made about efficiency, accessibility and quality in health care (Beyens \& Bolt, 2017; Bolt, 2015). In my analysis of the epistemic practices of the National Health Care Institute, I encountered EBM in different forms: 1) the Institute used the hierarchical classification of medical knowledges (dominated by the RCT) to make reimbursement decisions; 2) 'evidence-based' quality indicators played a central role in the Institute's work with regard to quality management; 3) clinical guidelines played an important part in the Institute's work of identifying and tackling low-value care practices covered from the basic benefits package. In all these cases, the logic of EBM was employed to counteract cognitively biased or prejudiced outcomes in reimbursement decisions, quality management or disinvestment efforts.

There are three major findings from my studies of EBM in the Institute's epistemic practices that I will address in the next pages. First, I found that the logic of EBM plays a central part in objectifying and justifying complex health policy decisions. But while EBM is generally thought to avoid biased, discriminatory or prejudiced outcomes in decision-making, I found that the logic of EBM comes with its own challenges regarding the issue of epistemic injustice. Second, I found that epistemic injustices become visible and perceptible through the work of the Institute, but that these injustices do not necessarily originate in the work of the National Health Care Institute itself, but rather in the wider knowledge infrastructure from which the Institute draws in its decision-making processes. Third, I found that the Institute itself struggles to reconcile the formal rationality of EBM with the messiness of real-life decision-making. I witnessed the Institute's 'tinkering' with regard to EBM: the work required to make EBM 'fit' the Institute's decision-making reality and reconfigure EBM's working methods in order to better serve 'impartial', 'objective' and 'just' decision-making in particular contexts. Next, I will elaborate on these three findings.

\section{1 | EBM in Decision-Making Practices}

The different cases described in this book illustrate how EBM's knowledge hierarchy, evidencebased quality metrics and clinical guidelines help decision-makers to objectify and justify complex health policy decisions. Chapter 1 shows that the National Health Care Institute justifies public spending on health care provisions by, first and foremost, assessing a treatment's clinical effectiveness. If a treatment is not demonstrably effective, it is not eligible for reimbursement from social health insurance. In assessing a treatment's clinical effectiveness, the Institute employs EBM's hierarchical classification of types of medical knowledge, with the RCT valued higher than e.g. observational designs. In EBM the RCT is considered to be the ultimate research instrument that provides 'objective' knowledge about a treatment's effect: the RCT's experimental design is thought to protect against sources of bias and possible confounders. As such, the hierarchical classification 
of medical knowledges provides the Institute with a kind of logic of knowledge consumption for making reimbursement decisions that are justifiable in the public arena as 'objective', 'impartial' and 'just'.

In Chapter 2 we saw that insurers justified their plans to centralize Dutch emergency care in a few specialized centers by referring to quality indicators that were based on clinical guidelines, RCTs and systematic reviews. Although quality indicators are not directly linked to the clinical literacy movement that EBM originally set out to be, some have called indicators a 'branch' of EBM (Eddy, 2005) as they follow the same logic: clinical science can determine 'what works' and parameters based on these scientific findings can form an 'objective' or 'impartial' standard for provider behavior (Tanenbaum, 1995, 2012). It is the task of health care insurers in the Netherlands to safeguard both quality and (budgetary) efficiency in social health insurance by stimulating competition between health care providers, e.g. through selective contracting. To facilitate selective contracting, insurers require information about the performance of different health care providers that allow making a conscious choice between them. The case reported in Chapter 2 shows how insurers proposed to use quality indicators as an 'impartial' and 'objective' standard to evaluate the performance of different hospitals in providing emergency services in order to contract selectively between them.

The case reported in Chapter 3 shows that, in cooperation with field parties, the National Health Care Institute took a quantitative approach to identifying overuse, underuse and misuse of medical services covered from social health insurance. Clinical guidelines played an important part in this approach. In the 'Appropriate care' program, the Institute, together with clinical experts and field parties, aimed to find overuse, underuse and misuse of medical services by comparing the norms for clinical practice as stated in clinical guidelines, with administrative data recording what actually happened to patients in practice. The combination of standards formulated by the professions themselves and administrative data recording actual practice, substantiated suspicions that something was going on (or going wrong) in certain health care practices. As such, the 'Appropriate Care' program 'objectified' or 'quantified' the gap between knowledge and practice, making the issue of 'waste' in public health care expenditure visible and actionable in the public arena.

The cases reported in this book show that the logic of EBM (its hierarchy of medical knowledges, its clinical guidelines and its derived quality metrics) are employed to counteract biased, discriminatory or prejudiced outcomes in policymaking and to justify such complex decisions in the public arena. At the same time, the different cases show that the use of EBM in decision-making was, nonetheless, challenged by patients (Chapter 1) and by medical professional organizations and local health care providers (Chapter 2) for bringing about 'unfair' policy outcomes.

Chapter 1 reported on a lawsuit in which patients challenged the National Health Care Institute's use of the principles of EBM in making reimbursement decisions. In particular, they challenged the dominant notion of 'objective' knowledge in the EBM-method. According to patients, the method attributed too little credibility to the experience of doctors and patients in determining the effectiveness of a treatment. Patients disputed, furthermore, whether 'hard evidence' (RCT) of clinical effectiveness can reasonably be required if patient groups are highly heterogeneous or 
when there is a lack of financial resources or research interest. The patients' court case against the method of EBM questioned whether these patients (and their doctors) had had a fair chance as credible and intelligible epistemic agents to influence the Institute's EBM-formatted decisionmaking process (Chapter 1 and 4).

Chapter 2 examined the notable resistance of medical professional organizations and local doctors and hospitals to insurers' plans to use 'evidence-based' quality metrics to reorganize Dutch emergency services. According to medical professionals, the quality indicators were imposed on them by insurers. They also claimed that insurers' quality standards were inadequate and inappropriate for measuring the quality of emergency services and facilitating a judicious choice between which centers should and which should not provide such services. With the logic of EBM as the foundation of quality management in health care, the focus is on standardized and quantified 'protocol' quality. Chapter 2 showed that, while quality metrics are typically presumed to guarantee impartiality and objectivity in comparing the quality of health services, the uncritical use of quality metrics potentially silences local, contextual and embodied forms of knowledge that are actually required for the proper use of such metrics. The dispute about quality indicators invited reflection on the question of whether quality indicators still serve to promote the quality of care if local field parties are neglected as epistemic agents in indicator-formatted decision-making processes (Chapters 2 and 4).

The rejection of EBM's logic for complex health care decision-making recorded in Chapters 1 and 2 shows that, though EBM might be employed to justify decisions explicitly and avoid biased outcomes, it is questionable whether 'evidence-based' decision-making actually avoids epistemic injustices from occurring in policymaking practice (Chapter 4).

\section{2 | Infrastructural Dependence}

In Chapter 4 we saw that Fricker's concept of 'epistemic injustice' provides valuable insights in analyzing policy practices (Fricker, 2007). For example, it shows that the embodied knowledge of patients and doctors is sometimes marginalized as credible or useful information within the EBM-method. It illustrated, furthermore, that while it seems justified from a health-economics perspective to assess the health benefits between different treatments by looking at 'objective' quantitative population-based research in order to make a conscious choice for coverage from social health insurance, the fact that that certain groups of patients lack the opportunity to provide such quantified evidence can potentially create an unfair epistemic disadvantage for this group in reimbursement decisions ${ }^{1}$. It showed, moreover, that the wrongful use of quality metrics based on standardized, quantified medical knowledge can potentially (unjustifiably) silence and overrule the embodied, contextual knowledge of local professionals.

\footnotetext{
1 This is one of the reasons why the National Health Care Institute works with conditional reimbursements: it can include promising treatments in the insured package - for a specific period of time - that do not yet fulfil the criteria for reimbursement, on condition that during that period data are collected on the clinical effectiveness of the treatment (Boon, Martins, \& Koopmanschap, 2015; College voor Zorgverzekeringen, 2012).
} 
Chapter 4 also showed that the concept of 'epistemic injustice' is not able to fully grasp how 'knowing gets done' in public decision-making. More specifically, it fails to provide understanding of how epistemic injustices operate not only on the level of interaction between groups of knowers, but also on the level of epistemic infrastructures that determine epistemic discourses beyond these groups. The term 'infrastructure' is used to refer to something that runs beneath an actual structure; it is that upon which something else rides, or works: such as railroad tracks for train transport or cable wires for television broadcasting (Star \& Bowker, 2006). In STS, the concept has been used to analyze all kinds of information infrastructures, showing, for example, how the identification of diseases came to 'run' on the International Classification of Diseases (ICD) (Bowker \& Star, 1999); how scientific 'objectivity' in psychological and medical research came to 'run' on control group comparison (Dehue, 2000; Dodier \& Barbot, 2008) and how EBM became the 'gold standard' upon which health care 'runs' (Timmermans \& Berg, 2003). STS studies of informational infrastructures have shown that decades of negotiations often inform and shape such knowledge conventions, and how they silently become embodied in the (health care) environment and in notions of 'good practice'.

One of the major findings of my research is that for the execution of its tasks with regard to organizing the accessibility, efficiency and quality of health care, the Institute relies profoundly on a wider network of knowers, knowledge conventions and knowledge products. The scientific methods, dominant notions of 'evidence' and epistemic ideals of 'objectivity' that the Institute employs to attain impartiality and justice in its decision-making processes, are often not invented within the Institute itself. Chapter 1 showed that the Institute draws on the logic of EBM that, since the mid-1990s both nationally (Bolt, 2015) and internationally, is considered 'the' knowledge philosophy in both clinical practice and policymaking (Dobrow et al., 2004; Parkhurst, 2017). The Institute's use of the epistemic ideal of 'objectivity' in EBM is based on the fact that this is an international knowledge standard that is the result of years of negotiations amongst health care professionals, policymakers, payers and patients (Bolt, 2015; Timmermans \& Berg, 2003). Similarly, Chapter 2 showed that insurers draw on the logic of EBM that has, over recent decades, become the foundation of the quality movement (Eddy, 2005; Tanenbaum, 1995, 2012). Chapter 3 showed how, for its systematic screening of the provisions covered in the Dutch basic benefits package, the Institute draws on the International Classification of Diseases (ICD), which has served as an international standard for the identification of diseases since the 1900s (Bowker \& Star, 1999). These are all examples of existing national and international knowledge conventions that define and format the Institute's decision-making processes.

The Institute relies, furthermore, on data and information that is readily available in the field, and, thus, on the agenda setting and funding mechanisms in national and international medical research, on priorities in the development of standards and protocols and on dominant medical discourses. The different chapters of this book showed how the Institute depends, for the execution of its legal tasks, on the availability of quantified, standardized knowledge about clinical practice as represented in RCTs, systematic reviews, clinical guidelines and quality metrics. This means that 
the Institute, inevitably, relies on the priorities of external parties in developing clinical guidelines, quality standards ${ }^{2}$ and clinical studies that is increasingly. While my studies show that the Institute is a critical consumer of the knowledge produced by external parties, the fact that the Institute largely depends on the external production of RCTs, clinical guidelines and quality indicators makes its decision-making processes vulnerable for incorporating epistemic improprieties that have originated elsewhere.

Think, for example, about the research agenda for clinical studies that is increasingly set by private pharmaceutical and medical devices industries that undeniably work from a different set of priorities and values than the public National Health Care Institute. Pharmaceutical companies are nowadays in a position to prioritize medical research topics. They frequently conduct large trials that investigate the (often marginal) health gains 'in near saturated fields of research', e.g. involving complex medication regimes in the treatment of cancer (Greenhalgh, Howick, \& Maskrey, 2014), while other fields of medical research receive little research attention and funding, and remain terra incognita (see also Chapter 1 ). Pharmaceutical companies not only decide which treatments will be compared in empirical studies, they also determine the outcome measures for establishing effectiveness and are furthermore in a position to influence the outcome of studies (e.g. by overpowering trials to make sure that small differences will be statistically significant and by influencing inclusion criteria to select a research population that is most likely to respond to treatment) (Greenhalgh et al., 2014)3. Most clinical research has 'minimal patient input', which often means that they reflect outcomes that matter to researchers, rather than those that matter to patients (Greenhalgh et al., 2015, p. 2; Wiering, de Boer, \& Delnoij, 2017). It is, furthermore, still a topic of research how much influence patients have as epistemic agents in the setting of quality standards and clinical guidelines (M. Armstrong, Mullins, Gronseth, \& Gagliardi, 2018). So, the epistemic practices of external parties that the Institute inevitably relies upon raise their own set of questions regarding epistemic injustice.

In short, Fricker's notion of epistemic injustice focuses mostly on the level of interaction between groups of knowers, and much less on the level of epistemic infrastructures that determine epistemic discourses beyond these groups. Yet, the Institute draws heavily from an existent knowledge infrastructure of national and international medical conventions, professional standards, clinical literature, data, medical research agenda's and funding mechanisms. What would the responsibilities of the National Health Care Institute look like with regard to the epistemic inequalities that are inherent in the knowledge infrastructure to which it inevitably resorts? What do

\footnotetext{
2 The Institute does have the legal authority (the 'Meerjarenagenda', an authoritative knowledge agenda for the development of quality information in health care) to impose priorities in guideline development or in the development of quality indicators. However, this authority is used only in exceptional circumstances.

${ }^{3}$ Sometimes, the power of contemporary pharmaceutical companies even goes so far that they determine what counts as a 'disease' or 'disorder', for example, 'female sexual arousal disorder' to be treated with sildenafil (Basson, Mclnnes, Smith, Hodgson, \& Koppiker, 2002) or 'male baldness' to be treated with finasteride (Leyden et al., 1999).
} 
the virtues of 'epistemic justice' require of social institutions in this regard (Anderson, 2012)? And what does epistemic responsibility look like in the collective context of a knowledge infrastructure (cf. Isaacs, 2011)? While Fricker provides a valuable concept for recognizing epistemic injustices, she leaves us with questions regarding the responsibilities to deal with this type of injustice in the public context of knowledge infrastructures (see also Dieleman, 2015).

\section{3 | Heterogeneity \& Tinkering}

My studies at the National Health Care Institute, furthermore, show heterogeneity in its epistemic practices: on the one hand the Institute employed EBM's hierarchy of medical knowledges as a logic for knowledge consumption in making national reimbursement decisions (Chapter 1), while on the other hand an expert committee - installed at the Institute to assess the 'evidence-base' of insurers' quality indicators - questioned the (uncritical) use of the logic of EBM as the principle foundation of quality management, and emphasized the need to supplement quantified quality metrics with local, contextual and embodied forms of knowledge (Chapter 2). While at first sight this may seem like an inconsistency in epistemic practices, in my four years of research at the Institute, I learned to look at the heterogeneity in the Institute's epistemic practices in a different way. The Institute has diverging legal tasks relating to package management and quality management. While the logic of EBM helps to objectify and justify public decisions with regard to accessibility, efficiency and quality of health care, at the same time the usefulness of the EBM-logic is contingent to the context in which the different types of decisions are made.

The different chapters of this book show the Institute's struggle to reconcile the unambiguous formal rationality of EBM with the 'messiness' of the practical rationality of decision-making in diverging contexts (Jenkings \& Barber, 2004, p. 1765; Russell \& Greenhalgh, 2012). Being at the Institute for four years allowed me to witness the 'tinkering' that was done (Knorr Cetina, 1981; Mol, 2008) to make the logic of EBM fit complex decision-making processes. In STS, the term 'tinkering' is used to describe the mediation work that is required to make functional technologies or formal knowledge standards fit real-life practices that are 'full of complex ambivalence and shifting tensions' (Mol, Moser, \& Pols, 2015, p. 14; see also Timmermans \& Berg, 2003). EBM, rather than a set of general rules, is adaptable and in need of adaptation - 'tinkering' - in order to provide 'good care' or 'good policymaking' (see e.g. Timmermans \& Berg, 2003).

It is this type of mediating work that I observed in the Institute's epistemic practices. Chapter 1 showed, for example, that the Institute, aware of the shortfalls of EBM's strict hierarchy, started to experiment in reimbursement decision-making with 'GRADE' - an EBM-method that allows more flexibility within the original hierarchy of medical knowledges (see Guyatt et al., 2008). The Institute has, furthermore, drawn up an 'appropriate evidence questionnaire', a guide to whether RCTs can reasonably be expected in specific reimbursement decisions (Heymans, Kleijnen, \& Verstijnen, 2013). It is also exploring how to use 'real-world data' (Makady et al., 2017) and patient experiences from social media in health technology assessments (Kalf, Makady, Ten Ham, Meijboom, \& Goettsch, 2018). 
Furthermore, in Chapter 2 we saw how the Institute assessed insurers' quality indicators as largely 'evidence-based' and, at the same time, emphasized the need for dialogue with local parties and the exploitation of contextual knowledge of local health professionals. While the National Health Care Institute is lawfully tasked with providing unambiguous, 'objective' quality standards, at the same time, we saw how the Institute stressed the necessity of evaluating the applicability of such standards in specific regional contexts that may require different priorities.

In Chapter 3, we witnessed how the Institute is learning to involve stakeholders in its quantitative effort to identify and tackle low-value care practices. The Institute goes back and forth between field parties and the quantified analysis of overuse, underuse and misuse of medical services that surface from comparing clinical guidelines with administrative data. The Institute's iterative investigative process resulted not only in calls for better guideline compliance, but equally in calls for the adjustment, revision or development of clinical guidelines.

In all these examples, the formal logic of EBM - i.e. that the RCT provides the most 'objective' knowledge for decision-making, that quality metrics and clinical guidelines from an 'objective' standard to evaluate provider behavior - is being adjusted, 'tinkered' with, contextualized.

Quantitative procedures are designed to facilitate the conscious exercise of counteracting bias and to prevent discriminatory outcome in policymaking (Porter, 1995). Evidence-based decision-making centers on the idea that there is a 'technocratic fix' for the legitimacy problem that accompanies most complex policy decisions (Syrett, 2003). From my ethnographic studies, I learned that the Institute has long moved away from an 'early naive faith' in the ability of EBM to straightforwardly guide complex health care decisions (Russell, Greenhalgh, Burnett, \& Montgomery, 2011, p. 2). I witnessed the Institute's 'tinkering' with EBM and related quantitative procedures. These adjustments seemed to be the result of a general sense at the Institute that the formal logic of EBM in itself does not necessarily create epistemically 'just' policy outcomes. The continuous 'tinkering' in epistemic practices illustrates that policymakers continue to search for ways of policymaking that meaningfully combine the standard, aggregate and quantitative knowledge of RCTs, indicators and guidelines with patients' and professionals' embodied, local and contextual knowledges.

The Institute's continuous efforts to improve policymaking practices also target the wider national and international knowledge infrastructure upon which the Institute depends. For example, the Institute is in constant dialogue with the drug and medical devices industries regarding health technology assessment (HTA) and reimbursement decisions. In these interactions the Institute addresses e.g. issues regarding the treatments compared in clinical studies, questions regarding outcome measures for establishing effectiveness, problems with overpowered or underpowered trials, doubtful inclusion criteria for the research population, et cetera. As such, the Institute critically consumes the research products produced in the wider knowledge infrastructure upon which it inevitably depends. The Institute also grants a subsidy for promising care treatments and medical devices to help finance research into the (cost-) effectiveness of these treatments or devices that is required for assessing their eligibility for reimbursement from public funds. The 
Institute is, furthermore, an active member of EUnetHTA, a network of HTA agencies, research institutions and health ministries across Europe that aims to develop reliable, timely, transparent and transferable information for public national HTAs in European countries. The Institute collaborates with the European Medicines Agency (EMA), the European agency tasked with the evaluation and supervision of medicines, to discuss e.g. outcome measures for drug evaluations that are relevant to the Institute's task of organizing the accessibility, efficiency and quality of health care. The Institute, moreover, holds periodical meetings with (third party) health care payers in other countries to discuss issues concerning, for example, expensive medicines and how to guarantee sustainable access to them. What's more, the Institute has structural dialogue with ZonMw, the Netherlands Organization for Health Research and Development. It does so, amongst other things, to address pressing knowledge gaps and to develop required knowledge products that the Institute finds lacking from the perspective of its legal tasks. The Institute, furthermore, maintains an authoritative knowledge agenda on quality information ('Meerjarenagenda') that can impose priorities in guidelines development and that holds external parties accountable for the development of quality standards requiring the involvement of patients in the construction process.

All these different network activities illustrate that the National Health Care Institute performs mediation work within the wider knowledge infrastructure in which it operates to make it better fit and facilitate decision-making processes in the changing material, financial, moral, social and political reality of the health care system.

\section{EXPERTS AND IDIOTS}

It is the epistemic practices of policymakers, and the possible epistemic injustice circulating in them, that is the topic of this book. The different chapters of this book show that while the logic of EBM is used to objectify and explicitly justify policy decisions, EBM's quantitative approach can lead to situations where groups of knowers are (unintentionally) epistemically marginalized. It also shows that, while instances of epistemic injustice might play out in decision-making processes, they do not always originate there, but rather in the wider national and international knowledge infrastructure from which decision-makers draw. I want to use these concluding paragraphs to address some of the remedies to which policymakers can turn in order to counteract instances of epistemic injustice in decision-making.

According to Fricker, there are three things that one can do to fight epistemic injustices (Fricker, 2012). The first remedy is to practice the 'virtues' of epistemic justice until they become automatic and habitual (see Box 1). Second, Fricker proposes the installation of structural mechanisms to prevent prejudicial bias: for example, blind review in academic publishing. Fricker's third remedy is 
to use cognitive dissonance ${ }^{4}$ as a catalyst to reflect on prejudice. Fricker believes that people can train themselves to use cognitive dissonance between their prejudicial perceptions of another's credibility/intelligibility and their conscious endorsement of non-discriminatory norms of judgement (Fricker, 2012). How useful are Frickers' remedies to prevent epistemic injustices in the context of policymaking?

\section{Box 1 | The Virtues of Epistemic Justice}

The 'virtue of testimonial justice' requires one to critically reflect on his or her possible operations of prejudice in judging the credibility of another speaker (Fricker, 2007, pp. 92-95). Drawing from Harper Lee's book "To Kill a Mockingbird", Fricker found testimonial injustice in the white jurors' failure to believe the testimony of Tom Robinson because they are prejudiced against black people (Fricker, 2007, p. 23). The 'virtue of testimonial justice' would require of the jurors to reflect on their prejudice and to, subsequently, reconsider their premature and biased dismissal of Robinson's testimony.

The 'virtue of hermeneutical justice' requires that one guards against prejudice by assessing the inarticulate struggles of a speaker to make sense of his or her experience as the result of the speaker's lack of collective interpretative resources rather than evaluating these struggles as epistemic deficiencies inherent to the speaker himself/ herself (Fricker, 2007, p. 169). As an example of hermeneutical injustice, Fricker mentioned Carmita Wood, 'a woman who suffers sexual harassment prior to the time when we had this critical concept, so she cannot properly comprehend her own experience, let alone render it communicatively intelligible to others' (Fricker, 2007, p. 1). The 'virtue of hermeneutical justice' would require charitable listening to the inarticulate experiences of Carmita to help make sense of these experiences, instead of writing them off as incomprehensible rambles.

\section{1 | Virtues of Epistemic Justice}

The first remedy: the virtues of epistemic justice. Reflecting on policymaking practices in social health insurance from the perspective of epistemic justice is important, because if (groups of) knowers are not recognized in their capacity as credible and intelligible epistemic agents in policy processes, these knowers are - as a result thereof - potentially deprived of their fair share of public resources and excluded from political deliberative practices in which collective futures are

\footnotetext{
${ }^{4}$ The term 'cognitive dissonance' (Festinger, 1957) refers to people's internal need for consistency and the mental discomfort a person experiences when he or she is confronted with contradictory ideas, values or beliefs.
} 
constructed. But, what would be required of the National Health Care Institute in practicing the 'virtues' of epistemic justice?

The Institute has to navigate a variety of testimonies from a wide range of sources, amongst them those of patients, experts and researchers. The Institute's use of the principles of EBM to evaluate the 'objectiveness' of different testimonies, serves as a much-needed logic of knowledge consumption to make aggregate decisions at a population level. Can the Institute reasonably be expected to assess all these different types of testimonies (comprehensible or not), to judge the credibility and intelligibility of all those epistemic agents on their personal merits? I think not. As critics noted, Fricker's account of the 'virtues' of epistemic justice lacks an explanation of who is responsible (in their private, professional, public or political lives?) - and when (in what situation?) - for taking on the role of the virtuous listener (Dieleman, 2015; Maitra, 2010). What do the virtues of 'epistemic justice' require of public institutions (see Anderson, 2012)?

Moreover, this concluding chapter showed that, in order to adequately address epistemic injustice, it is not only important that we recognize its occurrence, but also that we understand where it originates. Even if the Institute were to actively practice the virtues of epistemic justice and become aware of the biases in its credibility and intelligibility judgements, these biases may still be inscribed in the decision-making instruments and in the wider national and international knowledge infrastructure upon which the Institute depends. As such, epistemic injustices can be hard to counteract by a single agent operating in a wider knowledge infrastructure. Practicing the virtues of epistemic justice is an important starting point to generate awareness. In policymaking practices, however, the undoing of epistemic injustice requires more.

\section{2 | Structural Remedies}

Fricker's second remedy is to install structural mechanisms to prevent prejudicial bias - such as blind review in academic publishing (Fricker, 2012). The different cases presented in this book are witnesses of the structural remedies installed to standardize procedures in decision-making: i.e. the use of EBM's hierarchy of medical knowledges, quality metrics and clinical guidelines. EBM is generally supposed to serve as a structural remedy to avoid bias and prejudice in (medical) decision-making. At the same time, the different chapters showed that these procedures can bring about their own testimonial and hermeneutical injustices in policy outcomes and that it is questionable whether EBM always avoids bias or prejudice.

Structural remedies by themselves are never a guarantee for epistemically just outcomes in policymaking. Each structural remedy contains its own politics and prioritization. By the very fact that EBM functions as a knowledge standard in clinical practice and health care policymaking, it is inherently political because standards 'restructure the environment of which they become part' (Timmermans \& Berg, 2003, p. 27; Timmermans \& Epstein, 2010). Every knowledge standard, classification or epistemic convention - such as the RCT, a clinical guideline or quality indicator - decides 'what will be visible or invisible within the system' (Bowker \& Star, 1999, p. 44), making 
certain testimonies heard and certain issues actionable in the public domain while, at the same time, making others mute and in-actionable (Murphy, 2006).

This book shows that in order for structural remedies - such as the different manifestations of EBM - to facilitate 'just' social health insurance policy, they require constant vigilance, 'tinkering', mediation work and adjustments to the diverse decision-making contexts in which they operate. The identification and tackling of epistemic injustices, thus, requires fundamental reflection on policy processes, not only on policymakers' own prejudices, but on the prejudicial biases potentially inscribed in their epistemic instruments for achieving 'objectivity', 'impartiality' and 'justice' in decision-making.

\section{3 | Cognitive Dissonance and Idiocy}

A third remedy proposed by Fricker is to use cognitive dissonance as a catalyst to reflect on prejudice with regard to how knowing is done. Cognitive dissonance is the type of discomfort that Callon and Rabeharisoa experienced in their meeting with Gino. At the start of this concluding chapter, we witnessed how easy it is to dismiss Gino's refusal of medicine, genetic testing and the professional medico-social networks for LGMD as the actions of a backward, simple-minded and amoral man, as a patient in denial or as the strange attitudinal remnants of a troubled childhood. Sociologists Callon and Rabeharisoa, however, actively worked to protect Gino from such 'hasty judgements and deliver him from the stigmatization of which he is a victim' (Callon \& Rabeharisoa, 2004, p. 15).

Many have argued that Fricker's virtues of epistemic justice cannot reasonably be required (Alcoff, 2010; Langton, 2010; Sherman, 2016). This is due to the fact that prejudice is 'difficult to control even by the most conscientious and well-intentioned agents' as it often happens automatically and 'more rapidly than conscious thought' (Anderson, 2012, p. 167). Yet, sociologists Callon and Rabeharisoa show that it can be done by feeding off their uncomfortable contradictory beliefs and values that emerged from their encounter with Gino. Callon and Rabeharisoa continued to look for this unease: they went on searching for dissonant voices, conflicting ideas and contradictory values in order to uncover their own unexamined ways of knowing (Callon \& Rabeharisoa, 2004, p. 22).

This is the type of training Fricker proposes when she mentions the use of cognitive dissonance. It is also the type of work that Stengers proposes when she suggests we look for the conceptual persona of the 'idiot' (Stengers, 2005), to reflect on 'what we are busy doing' (Michael, 2012). If cognitive dissonance is the means to detect and address epistemic injustices at an individual level, then social conflict, public controversies and system refusals are the means to identify and combat epistemic injustices at the level of knowledge infrastructures. Moments of system-breakdown are windows of opportunity to learn about biases, prejudices and other epistemic wrongdoings in knowledge infrastructures.

The social advocacy work by gay and lesbian rights organizations in the 1980 s can serve as a rich example. When in 1980 the American Psychiatric Association (APA) planned to publish its widely used handbook for psychiatric diagnosing - the third Diagnostic and Statistical Manual 
(DSM 3) - it met the wrath of social advocacy groups for gay and lesbian rights. The fierce lobbying of these groups led to the de-classification of homosexuality as a psychiatric disease and ultimately - to the de-medicalization of the category of homosexuals in general (Kirk \& Kutchins, 1992). Around the same time, gay advocacy groups dismantled attempts made in San Francisco to combine standardized forms of insurance companies and data of the Census Bureau, as the gay community (that was confronted with the newly classified disease of HIV/AIDS) feared that the combination of these data would be used to 'disenfranchise young men living in San Francisco from health insurance' (Bowker \& Star, 1999, p. 44). Similarly, Epstein describes how the AIDS movement in the 1980s, through its capacity to garner credibility and a voice in the scientific world, actively shaped clinical research trials and, as such, transformed biomedical research practices to a remarkable extent (Epstein, 1998). It shows how social advocacy groups thoroughly disrupted the knowledge standard in clinical research, diagnostic and insurance practices by raising awareness of issues with regard to racial and gender profiling in health care and the reification of biological notions of difference between groups in clinical research (see also Epstein, 2007; Helberg-Proctor, 2017).

The recent refusal of the National Health Care Institute to reimburse expensive orphan drugs if pharmaceutical companies fail to be transparent about their prices (NOS, 2018) is another example of an act of disruption of the system. In the current Dutch social health insurance system, the Institute is expected to calculate the cost-effectiveness of expensive orphan drugs and to decide on their public reimbursement. It has to perform this task while the pharmaceutical industry generally refuses to be transparent about how the exorbitantly high prices of these drugs are established. In a recent newspaper article, the Institute's chairperson described the Institute's uncomfortable internal conflict between the duty to help people with a rare disease, and the task to look after the interests of 17 million citizens who pay the premiums for the seemingly unjustifiably high prices of orphan drugs (Financieel Dagblad, 2018). The Institute's refusal to continue their work while being epistemically deprived of information about price-calculations challenged the current hegemony of the pharmaceutical industry. As such, the Institute functions as an 'idiotic' catalyst, asking those who are in power uncomfortable questions about drug-pricing practices and - ultimately - pushing to disrupt a system that no longer seems to fit.

With regard to the epistemic injustices in knowledge infrastructure, we can learn a lot from contention, system breakdown and 'idiotic' refusals. Knowledge conventions and epistemic traditions are so much part of everyday life, so deeply embodied in the world as we know it, that they only become visible upon breakdown or when they become the object of contention (Bowker \& Star, 1999; Star \& Ruhleder, 1996, p. 113). These are the moments of a collective experience of cognitive dissonance with regard to public practices of knowing. The question is, can we systematically organize this type of learning or reflection?

In order to answer that question, let us look at recent attempts to include alternative voices in decision-making practices. Over the past decades we have witnessed how policymakers experiment with deliberative spaces to engage citizens directly in decision-making processes in order to include 
the voices of lay people, patients or 'the public', who might have an alternative perspective on a policy matter to that of experts. However, it has been shown that such 'invited publics' easily buy into classical expert models, established vocabulary and shared imaginaries with regard to the topic under debate (Felt \& Fochler, 2010; Wynne, 2007). Voice is, furthermore, often 'reduced to choice' and restricted to specific articulations of policy problems (Benschop, Horstman, \& Vos, 2003, p. 146). Therefore, concerns are raised whether this type of invited voice is (unintentionally) being 'made' or 'wrongly appropriated' in such 'formalized mechanisms of voice' (Felt \& Fochler, 2010; Irwin, 2006; Michael, 2012, p. 541).

This shows that attempts to learn from alternative viewpoints that are not presented in existent socio-cognitive formats might just as easily disable, rather than invite, voice and dissent. In order to truly learn and broaden perspectives, it is necessary to create space for a type of 'hesitant voice': tentative, slow and always still 'in the process of articulation' (Benschop et al., 2003, p. 150). Efforts to systematically incorporate dissonant voices in policymaking that do not allow for 'questions, silences, doubts, lulls in conversation, un-comfortability, etc.' (Benschop et al., 2003, p. 149) easily run the risk of 'sanitizing' much needed confusion, ambiguity and blurriness instead of encouraging it.

The term 'idiot' in this book refers to someone who with his confused presence (just like prince Lev Myshkin in Dostojevski's novel 'The Idiot') questions societal values, norms and conventions. It is not a 'stupid person' or 'a person of low intelligence' but the one who slows down the process of achieving consensus because he questions the legitimacy of the political idiom. The title of this book - 'Experts and Idiots' - refers to a symmetry in the things that we can learn from those who know the system best (trained and educated in its instruments and logics), and from those who refuse that very system's idiom, legitimacy or even its existence.

Inevitably, every epistemic tradition creates its own experts, its own idiots. Faced with complex policy problems, we rely heavily on experts, but frequently forget to listen to the hesitant, inarticulate or dissonant voices. And yet, those are just as valuable, because these are the voices that are most likely to inform us about alternative pathways, realities and futures. Knowledge societies have found ingenious ways to organize and exploit expertise, but are hardly equipped to feed off dissonance and idiocy. As the different cases in this book show, it is from 'idiots' who refuse the logic of knowing in policymaking that we can learn most about the epistemic improprieties that might circulate in our epistemic practices. Unfortunately, there is no way to systematically organize idiotic catalysts; they stumble upon our path accidentally. What needs to be organized is sensitivity to recognizing the 'idiot' and sensing the potential for learning. 


\section{REFERENCES}

Alcoff, L. M. (2010). Epistemic Identities. Episteme, 7(2), 128-137.

Anderson, E. (2012). Epistemic Justice as a Virtue of Social Institutions. Social Epistemology, 26(2), $163 v 173$.

Armstrong, D. (2002). Clinical autonomy, individual and collective: the problem of changing doctors' behaviour. Social science \& medicine (1982), 55(10), 1771-1777.

Armstrong, M., Mullins, C., Gronseth, G., \& Gagliardi, A. (2018). Impact of patient involvement on clinical practice guideline development: a parallel group study. Implementation science : IS, 13(1), 55-55.

Basson, R., Mclnnes, R., Smith, M., Hodgson, G., \& Koppiker, N. (2002). Efficacy and safety of sildenafil citrate in women with sexual dysfunction associated with female sexual arousal disorder. Journal of women's health \& gender-based medicine, 11(4), 367-377.

Benschop, R., Horstman, K., \& Vos, R. (2003). Voice Beyond Choice: Hesitant Voice in Public Debates About Genetics in Health Care. Health Care Analysis, 11(2), 141-150.

Beyens, N., \& Bolt, T. (2017). 'A Medical Doctor in Politics'. Els Borst-Eilers and the Rise of Evidence-Based Healthcare in the Netherlands. BMGN-Low Countries Historical Review, 132(1), 16-37.

Bolt, T. (2015). A doctor's order: the Dutch case of evidence-based medicine (1970-2015). AntwerpenApeldoorn: Garant.

Boon, W., Martins, L., \& Koopmanschap, M. (2015). Governance of conditional reimbursement practices in the Netherlands. Health Policy, 119(2), 180-185.

Bowker, G., \& Star, S. (1999). Sorting things out: classification and its consequences. Cambridge, Mass.: MIT Press.

Callon, M., \& Rabeharisoa, V. (2004). Gino's lesson on humanity: Genetics, mutual entanglements and the sociologist's role. Economy and society, 33(1), 1-27.

Carel, H., \& Kidd, I. (2014). Epistemic injustice in healthcare: a philosophial analysis. Medicine, Health Care and Philosophy, 17(4), 529-540.

College voor Zorgverzekeringen. (2012). Rapport: Voorwaardelijke toelating/financiering van zorg. Retrieved from Diemen:

de Jonge, H., \& Nijland, Y. (2018, 21 November 2018). 'Gegoochel met cijfers geluidsoverlast Lelystad Airport'. NOS. Retrieved from https://nos.nl/nieuwsuur/artikel/2260277-gegoochel-met-cijfers-geluidsoverlast-lelystadairport.html

de Zeeuw, H. (2017, 17 October 2017). Fouten in rapport naar geluidshinder van Lelystad Airport. NRC Hande/sblad. Retrieved from https://www.nrc.nl/nieuws/2017/10/17/vliegverkeer-rekenfouten-in-rapport-naargeluidshinder-van-lelystad-airport-13533394-a1577540

Deaton, A., \& Cartwright, N. (2018). Understanding and misunderstanding randomized controlled trials. Social science \& medicine, 210, 2-21.

Dehue, T. (2000). From deception trials to control reagents: The introduction of the control group about a century ago. American Psychologist, 55(2), 264.

Dieleman, S. (2015). Epistemic Justice and Democratic Legitimacy. Hypatia, 30(4), 794-810.

Dobrow, M., Goel, V., \& Upshur, R. (2004). Evidence-based health policy: context and utilisation. Social science \& medicine, 58(1), 207-217. 
Dodier, N., \& Barbot, J. (2008). Autonomy and Objectivity as Political Operators in the Medical World: Twenty Years of Public Controversy about AIDS Treatments in France. SCIENCE IN CONTEXT, 21(3), 403-434.

Eddy, D. (2005). Evidence-Based Medicine: A Unified Approach. Health Affairs, 24, 9.

Epstein, S. (1998). Impure Science: AIDS, activism, and the politics of knowledge. Berkeley: University of California Press.

Epstein, S. (2007). Inclusion: the Politics of Difference in Medical Research: University of Chicago Press.

Evidence-Based Medicine Working Group. (1992). Evidence-based medicine. A new approach to teaching the practice of medicine. JAMA, 268(17), 2420-2425.

Ezrahi, Y. (1990). The Descent of Icarus: science and the transformation of contemporary democracy. Cambridge, Mass.: Harvard University Press.

Farías, I. (2017). An Idiotic Catalyst: Accelerating the Slowing Down of Thinking and Action. Cultural Anthropology, 32(1), 35-41.

Felt, U., \& Fochler, M. (2010). Machineries for Making Publics: Inscribing and De-scribing Publics in Public Engagement. Minerva, 48(3), 219-238.

Festinger, L. (1957). A theory of cognitive dissonance. Evanston: Row, Peterson and Company.

Financieel Dagblad. (2018, 19-02-2018). Farmaceuten onder druk om open te zijn over prijsvorming. Financieel Dagblad. Retrieved from https://fd.nl/economie-politiek/1241987/farmaceuten-onder-druk-om-open-te-zijnover-prijsvorming

Fricker, M. (2007). Epistemic injustice: power and the ethics of knowing. Oxford: Oxford University Press.

Fricker, M. (2012). Replies to Alcoff, Goldberg, and Hookway on Epistemic Injustice. Episteme, 7(2), 164-178.

Ghinea, N., Lipworth, W., \& Kerridge, I. (2015). Evidence, regulation and rational prescribing: the case of gabapentin for neuropathic pain. Journal of Evaluation in Clinical Practice, 21(1), 28-33.

Greenhalgh, T. (2002). Intuition and evidence--uneasy bedfellows? British Journal of General Practice, 52, 395-400.

Greenhalgh, T., Howick, J., \& Maskrey, N. (2014). Evidence based medicine: a movement in crisis? BMJ, 348, g3725.

Greenhalgh, T., Snow, R., Ryan, S., Rees, S., \& Salisbury, H. (2015). Six 'biases' against patients and carers in evidence-based medicine. BMC Medicine, 13, 200.

Guyatt, G., Oxman, A., Vist, G., Kunz, R., Falck-Ytter, Y., Alonso-Coello, P., \& Schünemann, H. (2008). GRADE: an emerging consensus on rating quality of evidence and strength of recommendations. BMJ, 336(7650), 924-926.

Guyatt, G., Sackett, D., Sinclair, J., Hayward, R., Cook, D., \& Cook, R. (1995). Users' Guides to the Medical Literature: IX. A Method for Grading Health Care Recommendations. JAMA, 274(22), 1800-1804.

Helberg-Proctor, A. (2017). (Un)doing ethnicity: analyses of the socio-scientific production of 'ethnicity' in health research in the Netherlands. Maastricht.

Heymans, J., Kleijnen, S., \& Verstijnen, I. (2013). Passend bewijs bij het bepalen van effectiviteit van interventies. NEDERLANDS TIJDSCHRIFT VOOR GENEESKUNDE, 157(17), 806-811.

Irwin, A. (2006). The Politics of Talk: Coming to Terms with the 'New' Scientific Governance. Social Studies of Science, 36(2), 299-320.

Isaacs, T. (2011). Moral responsibility in collective contexts. New York: Oxford University Press. 
Jenkings, K., \& Barber, N. (2004). What constitutes evidence in hospital new drug decision making? Social science \& medicine, 58(9), 1757-1766.

Kalf, R., Makady, A., Ten Ham, R., Meijboom, K., \& Goettsch, W. (2018). Use of Social Media in the Assessment of Relative Effectiveness: Explorative Review With Examples From Oncology. JMIR cancer, 4(1).

Kirk, S., \& Kutchins, H. (1992). The Selling of DSM: the rhetoric of science in psychiatry. New York: De Gruyter.

Knaapen, L. (2014). Evidence-Based Medicine or Cookbook Medicine? Addressing Concerns over the Standardization of Care. SOC4 Sociology Compass, 8(6), 823-836.

Knorr Cetina, K. (1981). The Manufacture of Knowledge: An Essay on the Constructivist and Contextual Nature of Science. Oxford: Pergamon Press.

Knorr Cetina, K. (2007). Culture in global knowledge societies: knowledge cultures and epistemic cultures. Interdisciplinary Science Reviews, 32(4), 361-375.

Lambert, H. (2006). Accounting for EBM: Notions of evidence in medicine. Social science \& medicine, 62(11), 2633-2645.

Langton, R. (2010). Epistemic Injustice: Power and the Ethics of Knowing. By MIRANDA FRICKER. Hypatia, 25(2), 459-464.

Leyden, J., Dunlap, F., Miller, B., Winters, P., Lebwohl, M., Hecker, D., ... Draelos, Z. (1999). Finasteride in the treatment of men with frontal male pattern hair loss. Journal of the American Academy of Dermatology, 40(6), 930-937.

Maitra, I. (2010). The Nature of Epistemic Injustice. Philosophical Books, 51(4), 195-211.

Makady, A., ten Ham, R., de Boer, A., Hillege, H., Klungel, O., \& Goettsch, W. (2017). Policies for Use of RealWorld Data in Health Technology Assessment (HTA): A Comparative Study of Six HTA Agencies. Value in Health, 20(4), 520-532.

Michael, M. (2012). "What Are We Busy Doing?": Engaging the Idiot. Science, Technology, \& Human Values, 37(5), 528-554.

Mol, A. (2008). The Logic of Care: health and the problem of patient choice. London; New York: Routledge.

Mol, A., Moser, I., \& Pols, J. (2015). Care in Practice On Tinkering in Clinics, Homes and Farms.

Murphy, M. (2006). Sick building syndrome and the problem of uncertainty: environmental politics, technoscience, and women workers. Durham [N.C.]: Duke University Press.

NOS (Producer). (2018). Zorginstituut is 'chantage' door farmabedrijven zat. NOS. Retrieved from https://nos.nl/ artikel/2218154-zorginstituut-is-chantage-door-farmabedrijven-zat.html

Parkhurst, J. (2017). The Politics of Evidence: From evidence-based policy to the good governance of evidence. New York: Routledge.

Partos, R. (2014). No immigrants, no evidence? The making of Conservative Party immigration policy. Political Insight, 5(3), 12-15.

Porter, T. (1995). Trust in Numbers: the Pursuit of Objectivity in Science and Public Life. Princeton: Princeton University Press.

Russell, J., \& Greenhalgh, T. (2012). Affordability as a discursive accomplishment in a changing National Health Service. Social science \& medicine, 75(12), 2463-2471.

Russell, J., Greenhalgh, T., Burnett, A., \& Montgomery, J. (2011). "No decisions about us without us"? Individual healthcare rationing in a fiscal ice age. BMJ, 342(jun13 1), d3279. 
Sackett, D., Rosenberg, W., Muir Gray, J., Haynes, R., \& Richardson, W. (1996). Evidence based medicine: what it is and what it isn't. BMJ, 312(7023), 71-72.

Sherman, B. (2016). There's No (Testimonial) Justice: Why Pursuit of a Virtue is Not the Solution to Epistemic Injustice. Social Epistemology, 30(3), 229-250.

Star, S., \& Bowker, G. (2006). How to infrastructure. In L. Lievrouw \& S. Livingstone (Eds.), Handbook of new media: social shaping and social consequences of ICTs. London California New Delhi: Sage Publications; Thousand Oaks.

Star, S., \& Ruhleder, K. (1996). Steps toward an ecology of infrastructure: Design and access for large information spaces. Information systems research, 7(1), 111-134.

Stengers, I. (2005). The cosmopolitical proposal. In B. Latour \& P. Weibel (Eds.), Making Things Public (pp. 9941003). Cambridge, Mass.: MIT Press.

Syrett, K. (2003). A Technocratic Fix to the "Legitimacy Problem"? The Blair Government and Health Care Rationing in the United Kingdom. Journal of Health Politics, Policy and Law, 28(4), 715-746.

Tanenbaum, S. (1995). Getting there from here: evidentiary quandaries of the US outcomes movement. Journal of Evaluation in Clinical Practice, 1(2), 97-103.

Tanenbaum, S. (2012). Improving the quality of medical care: the normativity of evidence-based performance standards. Theoretical medicine and bioethics, 33(4), 263-277.

Timmermans, S., \& Berg, M. (2003). The Gold Standard: the challenge of evidence-based medicine and standardization in health care. Philadelphia, PA.: Temple University Press.

Timmermans, S., \& Epstein, S. (2010). A World of Standards but not a Standard World: Toward a Sociology of Standards and Standardization. Annual Review of Sociology, 36, 69-89.

Weisz, G., Cambrosio, A., Keating, P., Knaapen, L., Schlich, T., \& Tournay, V. (2007). The Emergence of Clinical Practice Guidelines. The Milbank Quarterly, 85(4), 691-727.

Wiering, B., de Boer, D., \& Delnoij, D. (2017). Patient involvement in the development of patient-reported outcome measures: A scoping review. Health Expectations, 20(1), 11-23.

Wynne, B. (2007). Public Participation in Science and Technology: Performing and Obscuring a PoliticalConceptual Category Mistake. East Asian Science, Technology and Society: An International Journal, 1(1), 99-110. 

Appendices

\section{Valorization}

Summary

Samenvatting

List of Publications

Acknowledgements

Author Details 



\section{Valorization of Research Insights}

\section{Societal Relevance, Impact and Practical Recommendations}

"Is that all?" asked Aglaya. "All? Yes,"said the prince, emerging from a momentary reverie. "And why did you tell us this?" - Fyodor Dostoyevsky, 'The Idiot' 


\section{VALORIZATION}

It is customary at the University of Maastricht to add to a dissertation an extra chapter that states the societal relevance of the study conducted, discusses its impact on public debates and provides practical recommendations for implementation. This chapter elaborates on the importance of reflecting on epistemic practices in policymaking and provides some practical tools for doing so. It also describes how the findings from my studies have been disseminated amongst both policymakers and health researchers and what these findings have 'done' in national professional debates and in international academic discussions. In this chapter I also report on my efforts to stimulate reflection on 'practices of knowing' not only in the field of health policy, but also in e.g. science journalism, (urban) sociology, cultural studies and the life sciences.

\section{WHY REFLECT ON EPISTEMIC PRACTICES IN (HEALTH) POLICY PRACTICES?}

In these times of soaring health care expenses and limited budgetary resources, policymakers charged with organizing the accessibility, efficiency and quality of health care face a daunting task. Their decisions easily become objects of social contention and public debate. Such decisions need to be well-substantiated and explicitly justified. The academic debate on prioritization in health care entails a long-established discussion about institutional processes, deliberative procedures and technical approaches for making such decisions (e.g. Daniels \& Sabin, 1997; Ham \& Roberts, 2003; Klein \& Maybin, 2012). Most of this academic work on prioritization in health care is prescriptive, focusing on how policymaking ought to be done.

This book takes a different approach. It is descriptive and reports how policymaking actually takes place in practice. It is an ethnographic exploration of the practices of using, assessing and warranting different types of knowledge to substantiate contentious policy decisions in the context of Dutch social health insurance. As such, this research adds to a body of qualitative research on prioritization in health care that focuses on the question how exactly scientific evidence, clinical expertise, patient experiences and stakeholder perspectives are brought together in the daily practice of making complex health policy decisions (e.g. Hodgetts, Elshaug, \& Hiller, 2012; Robinson, Williams, Dickinson, Freeman, \& Rumbold, 2012; Russell \& Greenhalgh, 2009). This type of empirical investigation enables reflection on the adequacy, appropriateness and social inclusiveness of policymakers' epistemic practices in decision-making.

Why is it important to reflect on epistemic practices in policymaking? Policymakers have come to rely heavily on quantified, technical and scientific knowledge to justify actions and to legitimize policy decisions as being 'impartial,' 'objective' and 'just' (Ezrahi, 1990; Porter, 1995). Such a technical approach to decision-making is presumed to prevent policymakers' cognitive biases from being triggered and its aim is to avoid discriminatory or prejudiced outcomes in policymaking. However, as 
numerous studies in the field of Science and Technology Studies have shown (Felt, Fouché, Miller, \& Smith-Doerr, 2017), practices of knowing are never neutral. Any epistemic tradition has its own preferred scientific methods, dominant notions of evidence and epistemic ideals of what counts as 'objective,' 'credible' and 'trustworthy' knowledge (Knorr Cetina, 1999, 2007; Murphy, 2006). As such, epistemic practices inevitably contain power relations, attributing 'credibility' to certain speakers (those who for example present the right kind of evidence, use the proper language, hold the required certificates or show other features that somehow are believed to guarantee a speaker's credibility) while the testimonies of other speakers are considered 'subjective' or even 'unintelligible' because they lack mastery of the privileged style of articulating, presenting and sharing knowledge.

According to philosopher Miranda Fricker, we can do a person (or a group of people) an injustice by not recognizing them in their capacity as a credible and intelligible knower. The term 'epistemic injustice' refers to 'a wrong done' to a person in his or her 'capacity as a knower', by the act of unjustifiably neglecting that person as an epistemic agent in his or her own right (Fricker, 2007, p. 1). The concept of 'epistemic injustice' raises particularly urgent questions in the field of policymaking: whose knowledge and what types of testimonies are actually counted as objective, credible and trustworthy contributions to policymaking processes? Someone who is not recognized in their capacity as a credible and intelligible epistemic agent in policymaking is not merely neglected as a knower in his/her own right, he/she - as a result of this - lacks the social power to influence public policy. If people lack the power to influence policy processes, they are potentially deprived of a fair share of public resources and are excluded from the deliberative practices of constructing collective futures.

In order to address potential epistemic injustices one has to become aware of epistemic inequalities between groups of knowers in their access to and representation in knowledgeintensive policymaking processes. That is why ethnographic research into policymakers' epistemic practices is such important work, as is reflecting on these practices from the perspective of epistemic injustice. The aim of this book is to instigate democratization of knowledge-intensive policy practices in health care so that different groups of knowers have equal opportunities to participate epistemically - as knowers - in policy practices. In order for that process to take flight, those working in the science-policy nexus must ask a set of critical questions regarding the working methods they employ in making public policy (see Box 1). Only by uncovering epistemic ideals in decision-making procedures and by critically reflecting on the societal consequences of these epistemic preferences, can policymakers uncover the epistemic injustices that potentially circulate in their work. 


\section{USING POLICY CONTROVERSIES FOR CRITICAL REFLECTION ON EPISTEMIC PRACTICES}

Apart from the societal purpose of studying epistemic practices in policymaking, the research reported in this dissertation was conducted to meet a practical knowledge need of the National Health Care Institute.

Building on almost 70 years of experience and expertise with regard to the statutory health insurance system, the Institute is an authoritative voice in the Dutch health care arena, ensuring that good quality health care services remain affordable and accessible to all citizens (Helderman, De Kruijf, Verheij, \& Van Thiel, 2014). Due to the public nature of the Institute, its knowledge work centers on the justification of decisions. While the Institute has increasingly legitimatized decisions through the scientization of the decision-making process (using evidence-based methodologies and robust scientific instruments), it nonetheless experiences difficulties explaining the foundations of these decisions to a wider public. The public outrage in 2012 that ensued the Institute's 'evidencebased' advice to stop the reimbursement of orphan drugs for the rare diseases of Pompe and Fabry is the most prominent example of this (Boon, Martins, \& Koopmanschap, 2015; Schinkelshoek \& Martini, 2012; Van den Brink, 2014). It showed that problems regarding the legitimation of difficult policy decisions in social health insurance are not always 'solved' by resorting to technical knowledge or scientific procedures.

In response to a growing recognition of the shortcomings of a technocratic approach in complex decision-making, the Institute set up a research project to investigate whether its knowledge strategies to justify public decisions are adequate in terms of representativeness and relevance, and how the Institute's knowledge strategies could potentially be improved in order to facilitate more socially robust policy decisions. This research project, called 'Public Legitimation of Social Healthcare Insurance in the Modern Risk Society', was funded and facilitated by the Institute and independently executed by researchers of Maastricht University. This book is the result of that collaborative research (see also Abrishami, 2018) and aims to find answers regarding the relevance and representativeness of the Institute's decision-making practices and how to create trust and social support for its decisions.

While public outrage about policy decisions or judiciary interference in decision-making are sensitive issues for the National Health Care Institute (whose authority and legitimacy lay in its acknowledged expertise and scientific standing), such uncomfortable and confusing events hold important clues for answering questions regarding the adequacy, relevance and representativeness of decision-making procedures. For a long time, technical approaches and formal procedures - for example evidence-based methodologies - have been considered remedies to avoid prejudice or cognitive bias in decision-making. However, my research shows that such technical approaches and formal procedures often contain biases and prejudices of their own. Disruptive events such as lawsuits and public controversies about decision-making procedures are actually touchstones for reflection on such biases and prejudices. Analyzing these events from the perspective of 
epistemic injustice (see Box 1) can hold the key towards creating more socially robust policymaking procedures.

\section{Box 1 | Epistemic (in)justice reflexivity tool}

\section{A Sample questions for policymakers to reflect on epistemic practices}

- What technical procedures do I use to justify policy decisions? What types of testimonies are preferred in these procedures?

- How do I assess/warrant/weigh the testimonies of different groups of knowers in formal decision-making procedures? What forms of knowledge do I recognize as credible and intelligible contributions in my decision-making procedures?

- Which social groups have (or lack) the epistemic resources to make themselves understood and heard in my decision-making procedures? Do different groups of knowers have equal opportunities to participate epistemically in the policy process?

- What might the societal consequence be of devaluing or excluding certain testimonies from policymaking processes?

- In cases involving epistemic injustice: what national and international networks exist in which epistemic procedures for decision-making are used and promoted? And what forums exist in which these procedures can be critically discussed and potentially improved?

\section{B Sample questions for health researchers to reflect on epistemic practices}

- What dominant notions of evidence and epistemic ideals of 'objectivity' do I use/ reproduce in my choice for a research method?

- What forms of knowledge and whose knowledge do I recognize as credible, intelligible and relevant in my research practice?

- In my research, what common-sense ideas do I use/reproduce about the trustworthiness and relevance of specific types of evidence, testimonies, sources of knowledge et cetera?

- What might the consequences be, in terms of representativeness of the researched phenomenon, of including/excluding particular testimonies, evidences, data, and information in my research practices?

In order to stimulate reflection and debate about the issues addressed above, I discussed the findings of my research in several internal debate forums at the National Health Care Institute (see Box 2). Sharing my research findings with policymakers at the Institute allowed me not only to inspire reflection on their epistemic practices, but also to check and refine my analysis of these practices with those who were directly involved. 


\section{Box 2 | Invited talks for policymakers}

Invited talk 'Understanding the knowledge dynamics in decision-making at the National Health Care Institute' held at a staff debate forum ['Zorgforum'] at the National Health Care Institute, February 2014

Invited talk 'Researching knowledge practices' held at a meeting of young professionals ['Jong ZIN'] at the National Health Care Institute, June 2014

Invited talk 'Understanding the knowledge dynamics in decision-making at the National Health Care Institute: the case of bladder instillations' held at a staff debate forum ['Zorgforum'] at the National Health Care Institute, October 2014

Invited talk 'Epistemic Culture at the National Health Care Institute: the case of bladder instillations' held at a staff debate forum ['Zorgforum'] at the National Health Care Institute, March 2015

\section{HOW TO STIMULATE REFLECTION ON EPISTEMIC INJUSTICES IN HEALTH RESEARCH?}

One of the major findings of my research is that policymakers rely profoundly on a wider network of knowers, knowledge conventions and knowledge products for making complex policy decisions. The scientific methods, dominant notions of 'evidence' and epistemic ideals of 'objectivity' that governing agencies such as the National Health Care Institute employ in decision-making processes are generally not invented by these agencies themselves. Existing national and international knowledge practices define and format the decision-making processes at a national policy level.

My studies show, for example, that policymakers rely on: a) clinical data and information that is readily available in the field; b) the agenda-setting and funding mechanisms in national and international medical research; c) priorities in the development of standards and protocols and d) dominant medical discourses. This means that policymakers' decision-making processes are vulnerable for incorporating epistemic improprieties that actually originated elsewhere.

Box 1 lists a set of critical questions that health researchers can use to reflect on their practices of producing professional guidelines, quality metrics, clinical research et cetera. It is essential to raise health researchers' awareness that if they fail to recognize epistemic injustices in research practices, there is a chance that epistemic inequalities generated in medical research are being reproduced in health policies that are based on these studies. In order to stimulate reflection in this regard, I sought opportunities to share and discuss my research insights and theoretical concepts not only with policymakers, but also with health researchers in national and international academic settings (see Box 3). 


\section{Box 3 | Invited talks and presentations for health researchers}

Invited talk 'Understanding the knowledge dynamics in decision-making at the National Health Care Institute: the case of bladder instillations' at the Tilburg School of Social and Behavioral Sciences (TRANZO) in September 2015

Presentation 'Evidence contested: An epistemic battle in healthcare rationing' at the Trading Zones in Technological Societies Conference, University of Liège, Belgium, October 2015

Invited talk 'Evidence-based Policymaking in a Post-Truth era?' at the Social Science \& Health Innovations Conference, Tomsk State University, Russia, May 2017

Poster-presentation and Pitch 'Evidence and doing "epistemic (in)justice" in prioritysetting' at the annual Research Day of the Care and Public Health Research Institute (CAPHRI), Maastricht University, November 2018

\section{IMPACT ON PUBLIC AND ACADEMIC DEBATES}

By discussing my research insights with policymakers and health researchers, and by publishing my work in (international) scientific journals (Box 4), I aimed to stimulate both professional reflection and academic debate about the democratic representativeness of epistemic practices in social health insurance, focusing specifically on the role of Evidence-based Medicine (EBM) therein. In particular my study of the lawsuit against the National Health Care Institute, reported in Chapter 1, received significant attention in professional and scientific media (Figure 1). Apart from its uptake in other scientific publications on prioritization in health care (e.g. Ettelt, 2018; Russell, 2017; van Overbeeke et al., 2018), the court case was discussed in national professional media (e.g. Van Maanen, 2016) and used as a case study in a widely discussed report of the Dutch Council for Health and Society called 'No Evidence Without Context. About the illusion of evidence-based practice in healthcare' (Raad voor Volksgezondheid en Samenleving, 2017). The case study has also been used for educational purposes for health researchers. Students at the Faculty of Health, Medicine and Life Sciences of Maastricht University study the case as part of the course 'Philosophy in Action'. As such, my study of the lawsuit has been used by a range of policymakers, health researchers, students and medical professionals to reflect on their practices of knowing. 


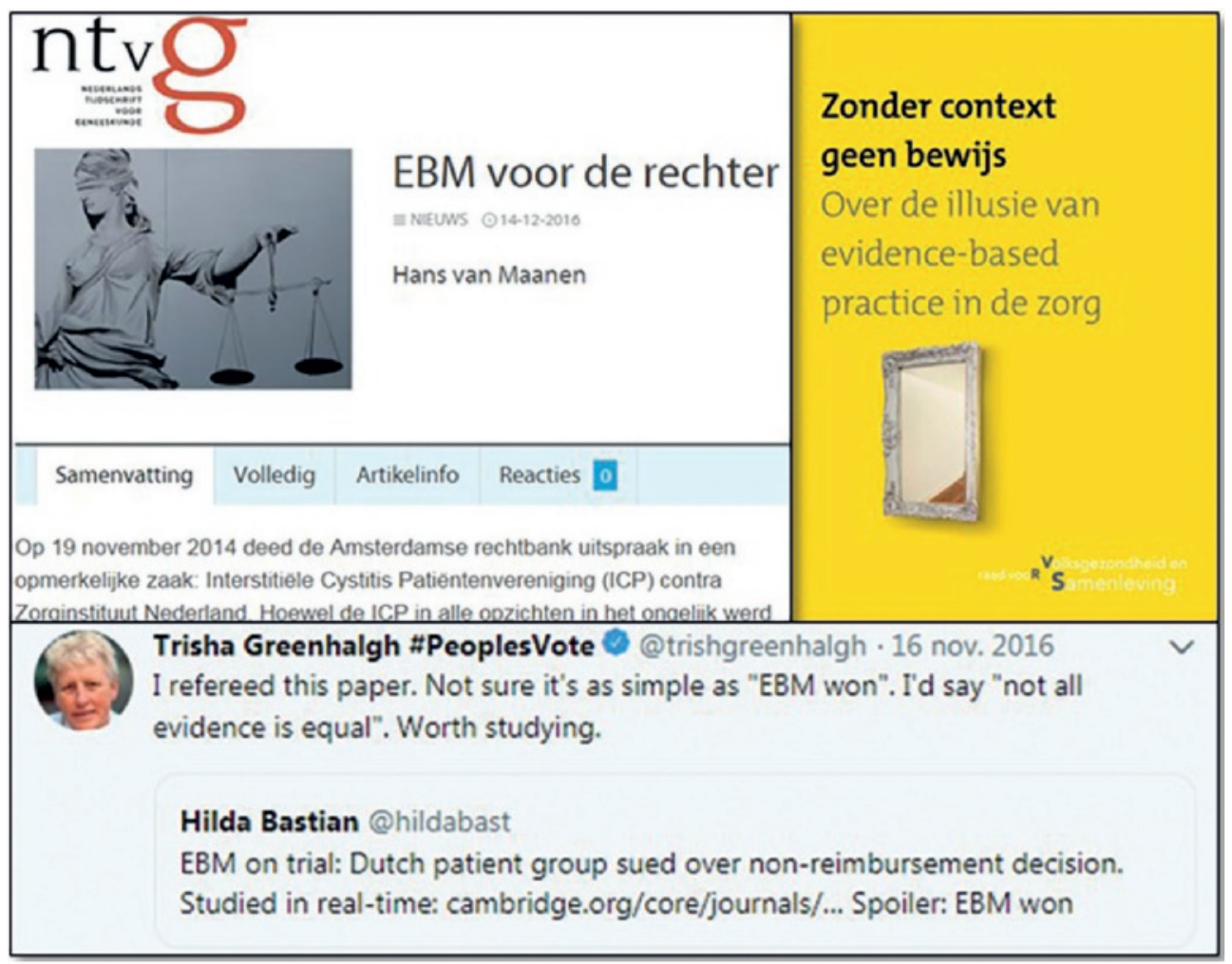

Figuur 1

\section{Box 4 | Publications in scientific journals}

Moes, F., Houwaart, E., Delnoij, D. and Horstman, K. (2016). Contested evidence: a Dutch reimbursement decision taken to court. Health Economics, Policy and Law, 12(3), 325-344

Moes, F., Houwaart, E., Delnoij, D. and Horstman, K. (2018). Strangers in the ER: Quality indicators and third party interference in Dutch emergency care. Journal of Evaluation in Clinical Practice,1-8.

Horstman, K. and Moes, F. (2018). 'Wat is dat eigenlijk, context?'. Tijdschrift voor Gezondheidswetenschappen (TSG, Dutch Journal for Life Sciences) 1/ 2018

Moes, F., Houwaart, E., Delnoij, D. and Horstman, K. (forthcoming). Collective Constructions of 'Waste': Epistemic practices for disinvestment in the context of Dutch Social Health Insurance. (revisions under review)

Moes, F., Houwaart, E., Delnoij, D. and Horstman, K. (forthcoming). Questions regarding 'Epistemic Injustice' in knowledge-intensive policymaking: two examples from Dutch health insurance policy. (revisions under review) 


\section{EPISTEMOLOGY, FILM \& TV: STIMULATING WIDER REFLECTION ON EPISTEMIC PRACTICES}

The standard view of science is that it is a neutral place where 'facts' are uncovered by disengaged scientists and where politics is absent. My studies have shown, however, that scientific research is never neutral and that there is no epistemic practice that does not - at the same time - privilege certain styles of reasoning and articulating testimonies while devaluing others. Insights from the field of Science and Technology Studies have shown that knowledge is man-made and value-laden (Latour \& Woolgar, 1979) and that there is no knowledge that does not presuppose and constitute power relations (Foucault, 1995). These are important insights, not only for policymakers, but for anyone involved in producing, using and/or reproducing scientific knowledge.

Apart from reaching out to policymakers and health researchers, I have, therefore, spent the past few years promoting deeper reflection on knowledge practices among a wide range of listeners: lay audiences, journalists, scientists and students ranging from Mathematics, Biology and Life Sciences to Arts, Culture, Sociology and Politics. For instance, when in 2015 Dutch citizens, researchers and industries were invited, within the context of the Dutch National Research Agenda $^{1}$, to submit research questions that were important from their personal, societal, scientific or industrial perspectives, I submitted two research questions. One of these questions - "Are we still asking the right kind of questions?", an attempt to raise awareness of the value of philosophical research and education within the business-oriented Dutch academic landscape - was selected by artist Inge Meijer to be part of a short creative film about the Dutch National Research Agenda. In that film I discussed the value of Philosophy of Science in general and Science and Technology Studies in particular with Prof. Trudy Dehue, a science-historian and sociologist of science.

Invited talks and public lectures formed another opportunity to promote deeper reflection on knowledge practices amongst a wider public. For example, I provided guest lectures on the epistemic work of scientists in a 'Science Journalism' course for journalists and in a variety of academic settings (see Box 5). On other occasions I made use of popular film and documentaries (e.g. The Wire, O.J.: Made in America) to show how epistemology works in day-to-day practices. Philosophy of Science is, generally, a compulsory (and often unpopular) part of academic curricula. In several public lectures I used the popular HBO's tv-series 'The Wire' to show how concrete epistemic practices - e.g. the production of crime statistics or the construction of court case 'evidence' - simultaneously shape and are shaped by city politics and social urban realities.

In particular the open lecture series on 'The Wire', held at the University of Amsterdam, received much attention and was widely covered in national media (see e.g. Kist, 2014; Kraak, 2014). Next to giving lectures, I published several articles on this topic for professional, academic

\footnotetext{
${ }^{1}$ A coalition of universities, university medical centers, technical colleges, the Royal Netherlands Academy of Arts and Sciences (KNAW), the Netherlands Organisation for Scientific Research (NWO) and the Netherlands Organisation for Applied Scientific Research (TNO)
} 
and lay audiences (see Box 6). The goal of the above lectures and publications was to show the transformative power of knowledge practices to arrange and rearrange contemporary societies. The use of popular film and documentary material allowed me to reach and engage a wide audience, and make them acquainted with the philosophical legacy of e.g. Bruno Latour and Michel Foucault, who have done groundbreaking work in dispelling the myth that facts are 'given' by nature and that knowing is a neutral and value-free activity.

\section{Box 5 | Invited talks on knowledge practices for a wider audience}

Invited talks 'Wetenschap ter discussie' for journalists in a science journalism course, organized by Stichting Cursussen Wetenschapscorrespondentie (SCW) in December 2013 and May 2014

Invited talk 'Kennispraktijken in The Wire', part of a series of public lectures at the University of Amsterdam, April 2014

Invited talk 'Wetenschap in transitie' for students of Biology, Natural Science and Mathematics in an elective course on the 'Circular Economy' at the Faculty of Science, University of Amsterdam, May 2015

Invited talk 'Bruno Latour \& The Wire', part of a series of public lectures organized by 'Philosophical Café' Felix \& Sofie, Amsterdam, December 2015

Invited talk 'Actor Network Theory \& The Wire' for master-students of Art, Culture \& Society in the course 'Advanced Sociology of Arts \& Culture' at Erasmus University Rotterdam, October 2018 
Box 6 | Epistemology, Film \& TV: Publications for professional, academic and lay audiences

Moes, F. (2014). 'All the pieces matter'- Latours slowciologie en The Wire. Sociologie. 10(3). 242-259

Moes, F. (2015) 'All the pieces matter'- Latours slowciologie en The Wire. In Van Oorschot, I., M'Charek, A., Harambam, J. \& Benschop, R. [red]. 'Monsters in de Sociologie. De wondere wereld van ANT. Boom/ Lemma, Amsterdam. 28-45

Moes, F. (2016). Blauwdruk van een omgekeerde wereld. Sociologie Magazine. 24(3), $10-11$

Moes, F. (2016). De stad in actie. Actor Netwerk Theorie. In Van Es, R \& Van Rossum, J. [red]. De stad als organisatie. Lessen uit The Wire. Deventer: Vakmedianet. 82-92

Moes, F. (2016). De 'Stat Game'. De verborgen krachten van de statistiek. In Van Es, R \& Van Rossum, J. [red]. De stad als organisatie. Lessen uit The Wire. Deventer: Vakmedianet. 134-150

Moes, F. (2017). Televisie voor Sociologen: 'O.J. Made in America' Sociologie Magazine. 25(1), 22-24. 


\section{REFERENCES}

Abrishami, P. (2018). Public Value of Medical Innovations: A quest for all and for all seasons. Maastricht University, Maastricht.

Boon, W., Martins, L., \& Koopmanschap, M. (2015). Governance of conditional reimbursement practices in the Netherlands. Health Policy, 119(2), 180-185.

Daniels, N., \& Sabin, J. (1997). Limits to Health Care: Fair Procedures, Democratic Deliberation, and the Legitimacy Problem for Insurers. Philosophy \& Public Affairs, 26(4), 303-350.

Ettelt, S. (2018). Access to treatment and the constitutional right to health in Germany: a triumph of hope over evidence? Health Economics, Policy and Law, 1-13.

Ezrahi, Y. (1990). The Descent of Icarus: science and the transformation of contemporary democracy. Cambridge, Mass.: Harvard University Press.

Felt, U., Fouché, R., Miller, C., \& Smith-Doerr, L. (2017). The handbook of science and technology studies. Cambridge; Mass: MIT Press.

Foucault, M. (1995). Discipline and punish : the birth of the prison. New York: Vintage Books.

Fricker, M. (2007). Epistemic injustice: power and the ethics of knowing. Oxford: Oxford University Press.

Ham, C., \& Roberts, G. (2003). Reasonable Rationing: International Experience of Priority Setting in Health Care. Maidenhead: Open University Press.

Helderman, J., De Kruijf, J., Verheij, J., \& Van Thiel, S. (2014). Dike-Reeve of the health care polder. A politicalsociological analysis of the realisation of the National Health Care Institute against a backdrop of a changing policy agenda and changing political-administrative and societal relations. Diemen: Zorginstituut Nederland.

Hodgetts, K., Elshaug, A., \& Hiller, J. (2012). What counts and how to count it: Physicians constructions of evidence in a disinvestment context. Social science \& medicine, 75(12), 2191-2199.

Kist, R. (2014, 7 april 2014 ). College over een tv-serie kan alleen met The Wire. NRC Handelsblad.

Klein, R., \& Maybin, J. (2012). Thinking About Rationing. London: King's Fund.

Knorr Cetina, K. (1999). Epistemic Cultures: How the Sciences Make Knowledge. Cambridge, Mass.: Harvard University Press.

Knorr Cetina, K. (2007). Culture in global knowledge societies: knowledge cultures and epistemic cultures. Interdisciplinary Science Reviews, 32(4), 361-375.

Kraak, H. (2014, 3 april 2014). Streetwise in science. ledereen loopt weg met de serie The Wire. Ook de Universiteit van Amsterdam. de Volkskrant.

Latour, B., \& Woolgar, S. (1979). Laboratory Life: the construction of scientific facts. Princeton (N.J.): Princeton University Press.

Murphy, M. (2006). Sick building syndrome and the problem of uncertainty: environmental politics, technoscience, and women workers. Durham [N.C.]: Duke University Press.

Porter, T. (1995). Trust in Numbers: the Pursuit of Objectivity in Science and Public Life. Princeton: Princeton University Press.

Raad voor Volksgezondheid en Samenleving. (2017). Zonder context geen bewijs : over de illusie van evidencebased practice in de zorg. Den Haag: Raad voor Volksgezondheid en Samenleving.

Robinson, S., Williams, I., Dickinson, H., Freeman, T., \& Rumbold, B. (2012). Priority-setting and rationing in healthcare: Evidence from the English experience. Social science \& medicine, 75(12), 2386-2393. 
Russell, J. (2017). The rationality of rationing: a rhetorical policy analysis of deliberations about resource allocation in the NHS.

Russell, J., \& Greenhalgh, T. (2009). Rhetoric, Evidence and Policymaking: a Case Study of Priority Setting in Primary Care (0068-1202). Retrieved from http://discovery.ucl.ac.uk/15560/1/15560.pdf

Schinkelshoek, J., \& Martini, T. (2012). Meer dan een Incident: CVZ, Pompe \& Fabry. The Hague: Schinkelshoek $\&$ Verhoog.

Van den Brink, R. (2014). Reimbursement of orphan drugs: the Pompe and Fabry case in the Netherlands. Orphanet Journal of Rare Diseases, 9(1), 017.

Van Maanen, H. (2016). EBM voor de rechter. NEDERLANDS TIJDSCHRIFT VOOR GENEESKUNDE (NVtG), 160:С3274.

van Overbeeke, E., Whichello, C., Janssens, R., Veldwijk, J., Cleemput, I., Simoens, S., . . . de Bekker-Grob, E. (2018). Factors and situations influencing the value of patient preference studies along the medical product lifecycle: a literature review. Drug discovery today.

Venturini, T. (2010). Diving in magma: how to explore controversies with actor-network theory. Public Understanding of Science, 19(3), 258-273. 

Introduction In liberal democracies, governments often justify their actions on the basis of scientific and technical knowledge. A solid foundation of public policy in scientific knowledge has become an expectation against which political actors are judged. This book explores how public accountability for thorny policy choices is constituted in policymakers' epistemic practices: their ways of using, assessing and warranting different types of knowledge to substantiate complex policy decisions. Whose knowledge and what types of testimonies are actually counted as relevant, credible and trustworthy contributions to policymaking processes? As such, this book critically engages with questions regarding the adequacy, appropriateness and social inclusiveness of policymakers' practices of knowing.

This book - which is based on four years of ethnographic study at the Dutch National Health Care Institute (Zorginstituut Nederland), an authoritative governing agency tasked to ensure the affordability and accessibility of good quality health care for all citizens - investigates epistemic practices in Dutch social health insurance. In times of soaring health care expenses and limited budgetary resources, policymakers charged with the organization of accessibility, efficiency and quality of health care face a daunting task. Their decisions easily become objects of social contention and public debate. Such decisions need to be well-substantiated and explicitly justified. The different chapters of this book each provide an in-depth empirical analysis of how the National Health Care Institute substantiates and justifies complex policy decisions, and reflect on the social representativeness and societal consequences of these epistemic practices.

Chapter 1 analyzes how the National Health Care Institute substantiated its decision against the reimbursement of bladder instillations with chondroitin sulphate or hyaluronic acid. In particular, the chapter discusses the ensuing lawsuit in which a specific group of bladder patients legally challenged the Institute for basing its decision primarily on scientific evidence from Randomized Controlled Trials (RCTs), thereby overruling clinical expertise and patients' experiences. The lawsuit centered on the question of whether the logic of Evidence-based Medicine (EBM) employed by the Institute, that emphasizes quantitative population-based research, is an adequate and fair method for making reimbursement decisions. Analyzing the court case in detail, this chapter engages with questions regarding the appropriateness and representativeness of the EBM-method in substantiating reimbursement decisions. It shows how inequalities in the distribution of epistemic resources between different groups of patients (for instance the availability of research funds), can potentially be reproduced in reimbursement decisions and, as a consequence, result in social inequalities in the distribution of health care provisions.

Chapter 2 addresses the Institute's epistemic work in quality management. It discusses a dispute between insurers, hospitals and doctors about a set of quality indicators for emergency care. Insurers planned to use the quality indicators to selectively contract emergency services 
and centralize Dutch emergency care in a few specialized hospitals. Insurers' plans caused much debate in the health care arena. Medical professionals questioned whether insurers' use of the indicators actually served to promote the quality of Dutch emergency care. Chapter 2 also explores the work of the National Health Care Institute in mediating this dispute. It engages with questions regarding the adequacy, representativeness and appropriateness of quality metrics as a tool for allocating resources in the health care landscape. While quality metrics are typically presumed to guarantee impartiality and objectivity in comparing the quality of health services, this chapter shows that the uncritical use of quality metrics potentially silences local, contextual and embodied forms of knowledge that are required for the proper use of such indicators.

Chapter $\mathbf{3}$ describes the epistemic work of the Institute in what was initially a 'disinvestment' initiative within the context of the Dutch basic benefits package. Faced with growing budget pressure, policymakers worldwide recognize the necessity of strategic disinvestment from ineffective, inefficient or harmful medical practices. The Institute developed a working method to identify the overuse, underuse and misuse of medical services currently covered from public funds. With this method the Institute, together with clinical experts and field parties, started comparing professional norms for clinical practice (as stated, e.g. in clinical guidelines) with the care actually delivered and paid for in practice (e.g. by looking at administrative data). Chapter 3 also engages with questions regarding the societal consequences of this type of knowledge work. It shows how the combination of clinical guidelines and administrative data makes the issue of 'waste' in public health care expenditure visible and actionable in the public arena and, as such, resets dependencies between payers, managers and medical professionals.

The first three chapters of this book illustrate that practices of knowing are never neutral. Policymakers work with specific notions of what counts as 'evidence' and particular ideas of what can be considered 'objective' knowledge. This means that epistemic practices inevitably contain power relations, attributing 'credibility' to certain speakers (those who present the right kind of evidence, hold the required certificates or show other features that are believed to guarantee a speaker's credibility) while the testimonies of other speakers are considered 'subjective' or even 'unintelligible' because they lack mastery of the privileged style of articulating, presenting and sharing knowledge.

Chapter 4 reflects on the Institute's epistemic practices from the perspective of 'epistemic injustice'. According to philosopher Miranda Fricker, we can do someone, or a group of people, an injustice by not recognizing them in their capacity as a credible and intelligible knower. The idea of 'epistemic injustice' seems particularly relevant in the context of policymaking. Someone who is not recognized in his/her capacity as a credible and intelligible epistemic agent lacks the social power to influence public policy. If people lack the power to influence policy processes, they are potentially deprived of a fair share of public resources and are excluded from deliberative practices of constructing collective futures. Chapter 4 shows how important it is to reflect on epistemic practices from the perspective of epistemic injustice. 
Research insights All in all, this book provides several valuable insights regarding epistemic practices in social health insurance. First of all, it shows that quantified and standardized forms of knowledge - the types promoted by EBM - play a central role in objectifying and justifying complex health policy decisions. At the same time, it shows that an uncritical use of quantified and standardized knowledge (including RCTs, clinical guidelines, quality indicators and administrative data) can actually lead to epistemic injustices in policymaking.

The study also shows that policymakers are generally aware of the shortcoming of quantified, standardized knowledge in decision-making. The first three chapters describe the Institute's continuous work to reconcile the formal rationality of EBM and standardized forms of knowledge with the messiness of real-life decision-making that is full of ambivalence, uncertainties and shifting tensions. Productive ways to complement quantified and standardized forms of knowledge with qualitative, embodied, local and experiential forms of knowledge in public decision-making are, however, still in the process of being developed.

Another important insight presented in this book is that policymakers heavily rely on existing national and international infrastructures of knowledge products and knowledge conventions. This means that policymakers are largely dependent on the priorities of external parties in setting research agendas, producing clinical studies, developing clinical guidelines, quality metrics and administrative data. Policymakers' external dependency makes their decision-making processes vulnerable for incorporating potential epistemic injustices that originate elsewhere. Think, for example, about the research agenda for clinical studies that is increasingly set by private pharmaceutical and medical devices industries that undeniably work from a different set of priorities and values than the public National Health Care Institute.

Conclusion In the concluding chapter, the author argues that addressing issues of epistemic injustice in decision-making practices requires more than reflecting on the work of policymakers alone. It would require a systems perspective at the level of the international knowledge infrastructure upon which policymakers rely. According to the author, the type of lawsuits, public controversies and social conflicts reported in this book are the primary means to identify and combat epistemic injustices at this level. These events of momentary system breakdown are windows of opportunity to learn about biases, prejudices and other epistemic injustices that might circulate in policymakers' epistemic practices and/or in the wider knowledge infrastructure from which they draw.

In this book, the 'idiot' refers to someone who disrupts systems, conventions and daily routines. Just like in Dostojevski's novel 'The Idiot', the term refers to a person who, with his confused presence, questions societal values, norms and conventions. It is not a 'stupid person' or 'a person of low intelligence' (as the term is often defined in modern dictionaries). In ancient Greece, the term 'idiot' referred to someone who did not speak the language of the polis. The 'idiot' is the one who slows down the process of achieving consensus because he questions the legitimacy of the political idiom. As the different cases in this book show, it is from 'idiots' who refuse the logic of knowing in policymaking that we can learn most about the epistemic injustices that might circulate in our epistemic practices. 



\section{Samenvatting}

Inleiding In een liberale democratie rechtvaardigen overheden hun besluiten voor een belangrijk deel op basis van wetenschappelijke en technische kennis. Deze kennis wordt namelijk verbonden met idealen van objectiviteit, onpartijdigheid en rechtvaardigheid in het maken van beleid. Dit boek onderzoekt hoe de publieke verantwoording van lastige beleidskeuzes wordt geconstrueerd in de kennispraktijken van beleidsmakers: welke verschillende vormen van kennis gebruiken ze om complexe beleidsbesluiten te onderbouwen en welke normen voor objectiviteit komen daarin tot uitdrukking? Wat voor soort en wiens kennis, bewijsstukken en getuigenissen gelden eigenlijk als relevante, geloofwaardige en betrouwbare bijdragen aan publieke besluitvormingsprocessen (en welke vormen van kennis juist niet)? Dit boek is een kritisch onderzoek van de dynamiek tussen kennis en beleid en reflecteert op de sociale representativiteit van deze kennispraktijken.

Dit boek richt zich met name op kennispraktijken in het Nederlandse sociale zorgverzekeringsstelsel. Het is gebaseerd op vier jaar etnografisch onderzoek bij Zorginstituut Nederland, een gezaghebbend instituut met de taak ervoor te zorgen dat gezondheidszorg van hoge kwaliteit betaalbaar en toegankelijk blijft voor alle Nederlanders. In de context van sterk oplopende kosten in de zorg en beperkte budgettaire middelen hebben beleidsmakers met de opdracht om de toegankelijkheid, doelmatigheid en kwaliteit van zorg te borgen een lastige taak. Beslissingen over verzekerde zorg en kwaliteit van zorg worden dan ook niet zelden onderwerp van publiek debat. Het is dus belangrijk om zulke beslissingen goed te onderbouwen. De verschillende hoofdstukken in dit boek bieden een empirisch-filosofische analyse van de manier waarop het Zorginstituut complexe beleidsbeslissingen onderbouwt en rechtvaardigt, en geven een kritische reflectie daarop.

Hoofdstuk 1 analyseert hoe het Zorginstituut de logica van Evidence-based Medicine (EBM) gebruikte om haar beslissing te rechtvaardigen dat blaasspoelmiddelen met chondroitïnesulfaat of hyaluronzuur voortaan niet meer vergoed zouden worden uit de nationale basisverzekering. Het Instituut vond, gebruikmakend van de EBM-methode, geen sluitend bewijs dat de middelen werken. Dit hoofdstuk beschrijft de rechtszaak die daarop volgde, waarin een groep blaaspatiënten het Zorginstituut verweet de visie van klinische experts en de positieve ervaringen van patiënten met dit middel naast zich neer te leggen, en haar besluit primair te baseren op wetenschappelijk bewijs uit gerandomiseerde studies (RCTs). In de rechtszaak stond daarmee de vraag centraal of de logica van EBM, en de nadruk die daarin ligt op kwantitatief populatieonderzoek, een goede methode is voor het maken van beslissingen over verzekerde zorg. Dit hoofdstuk geeft een diepteanalyse van deze rechtszaak en biedt daarmee een kritische reflectie op de EBM-methode in de onderbouwing van vergoedingsbesluiten. Het laat tevens zien hoe ongelijkheid in de onderzoeks-infrastructuur (bijvoorbeeld de allocatie van onderzoeksgeld onder specifieke problemen en/of patiëntengroepen) gereproduceerd kan worden in vergoedingsbeslissingen. Dit kan op haar beurt weer leiden tot sociale ongelijkheid in de verdeling van publieke voorzieningen. 
Hoofdstuk 2 gaat in op het kenniswerk van het Zorginstituut met betrekking tot de kwaliteit van zorg. Het analyseert een geschil tussen verzekeraars, ziekenhuizen en artsen over een reeks kwaliteitsindicatoren voor de spoedeisende zorg. Zorgverzekeraars waren van plan om deze indicatoren te gebruiken voor de selectieve inkoop van spoedzorg in Nederland en om de spoedzorg te centraliseren in een paar gespecialiseerde ziekenhuizen. Dit plan stuitte op stevige kritiek uit het veld. Medische professionals betwijfelden openlijk of verzekeraars' gebruik van de indicatoren de kwaliteit van de spoedeisende zorg in Nederland wel ten goede zou komen. Hoofdstuk 2 gaat over de manier waarop het Zorginstituut bemiddelde in dit geschil en reflecteert op de mate waarin kwaliteitsindicatoren adequaat en representatief gereedschap zijn als het gaat om de allocatie van voorzieningen en het inrichten van het spoedzorglandschap. Hoewel doorgaans verondersteld wordt dat kwaliteitsindicatoren een onpartijdige en objectieve maatstaf zijn voor het vergelijken van de kwaliteit van zorg, laat dit hoofdstuk zien dat het onkritische gebruik van zulke indicatoren, andere waardevolle vormen van kennis (lokale, contextuele, geleefde kennis) die nodig zijn voor passend gebruik van dergelijke indicatoren naar de achtergrond duwt.

Onder toenemende budgettaire druk erkennen beleidsmakers, overal ter wereld, de noodzaak om te stoppen met het financieren van ineffectieve, inefficiënte of zelfs schadelijk gebleken zorgpraktijken. Hoofdstuk 3 beschrijft het kenniswerk van het Zorginstituut in een dergelijk desinvesteringsinitiatief in de context van de nationale basisverzekering. Het Zorginstituut ontwikkelde een methode om met behulp van diverse nationale datasets over-, ondergebruik en misbruik op te sporen van zorgvoorzieningen die vergoed worden uit publieke middelen. Samen met klinische deskundigen en veldpartijen gebruikt het Zorginstituut deze methode om professionele normen voor zorg (bijvoorbeeld klinische richtlijnen) te vergelijken met de zorg die daadwerkelijk geleverd wordt in de praktijk (bijvoorbeeld door te kijken naar administratieve data van verzekeraars). Hoofdstuk 3 laat zien hoe het combineren van klinische richtlijnen en administratieve datasets het onderwerp van 'verspilling' in publieke zorguitgaven zichtbaar maakt en agendeert als publiek probleem. Het toont ook hoe dit leidt tot een herschikking van afhankelijkheidsrelaties tussen financiers, managers en professionals in de zorg.

De eerste drie hoofdstukken van dit boek laten zien dat kennispraktijken niet neutraal zijn. Beleidsmakers werken met specifieke opvattingen over wat telt als 'bewijs' en wat beschouwd kan worden als 'objectieve' kennis. Kennispraktijken brengen daardoor ook machtsrelaties met zich mee, omdat ze geloofwaardigheid toekennen aan bepaalde actoren (diegenen die het juiste soort bewijs presenteren, die de vereiste certificaten bezitten of andere eigenschappen tonen waarvan aangenomen wordt dat ze iemands geloofwaardigheid garanderen), terwijl de getuigenissen van andere actoren beschouwd worden als 'subjectief' of zelfs 'onbegrijpelijk' omdat zij niet voldoen aan de normen en conventies voor 'objectieve kennis'.

Hoofdstuk 4 reflecteert op het kenniswerk van het Zorginstituut vanuit het perspectief van 'epistemic injustice', een concept geïntroduceerd door de filosofe Miranda Fricker. Volgens Fricker kunnen we iemand, of een groep mensen, onrecht doen door ze niet te erkennen in hun hoedanigheid als 'kenner' op een bepaald gebied. Frickers idee van 'epistemisch onrecht' is relevant als het gaat 
om het maken van beleid. lemand die niet erkend wordt als een geloofwaardige bron van kennis heeft namelijk geen stem in beleidsprocessen en kan de uitkomsten ervan ook niet beïnvloeden. Zo iemand wordt uitgesloten van deliberatieve processen en heeft daardoor ook geen invloed op de manier waarop publieke middelen worden verdeeld. Hoofdstuk 4 laat zien hoe belangrijk het is om na te denken over kennispraktijken vanuit het perspectief van 'epistemische rechtvaardigheid'.

Conclusies Dit boek biedt belangrijke inzichten in de dynamiek van kennispraktijken in het Nederlandse sociale zorgverzekeringsstelsel. Ten eerste laat het zien dat gekwantificeerde en gestandaardiseerde vormen van kennis - het type kennis dat voldoet aan de norm van Evidencebased Medicine - een centrale rol spelen in het objectiveren en rechtvaardigen van complexe beleidsbeslissingen. Tegelijkertijd laat het zien dat het onkritische gebruik van dit type kennis (waaronder RCTs, klinische richtlijnen, kwaliteitsindicatoren en administratieve data) het risico in zich draagt van 'epistemische onrechtvaardigheid' in beleidspraktijken.

Het onderzoek laat ook zien dat beleidsmakers zich vaak bewust zijn van de tekortkomingen van gekwantificeerde en gestandaardiseerde kennis in besluitvorming en soms ook trachten dat te repareren. De eerste drie hoofdstukken beschrijven het continue werk dat het Zorginstituut verzet om de formele logica van EBM en gestandaardiseerde vormen van kennis te verzoenen met de weerbarstigheden, ambivalenties en spanningen van alledaagse besluitvormingsprocessen. De vraag hoe gekwantificeerde en gestandaardiseerde vormen van kennis goed gecombineerd kunnen worden met kwalitatieve kennis, lokale kennis, ervaringskennis en ander vormen van 'kennen' in de rechtvaardiging van publieke beslissingen, dient dan ook hoog op de agenda te staan.

Een ander belangrijk inzicht is dat het kenniswerk van beleidsmakers in de zorg sterk afhankelijk is van bestaande nationale en internationale infrastructuren van kennis. Dit houdt in dat de kennis die een rol speelt in beleid een specifieke, beperkte en selectieve inhoud heeft: beleidsmakers zijn afhankelijk van de prioriteiten van externe partijen bij de agendering van wetenschappelijk onderzoek, de productie van klinische studies, de ontwikkeling van professionele richtlijnen, kwaliteitsmeetinstrumenten en administratieve data. Impliciet zetten andere kennisinstituten, universiteiten, onderzoek fondsen, farmaceutische industrie en anderen, met hun onderzoeksprioriteiten en specifieke normen voor 'objectiviteit', dus een groot stempel op de wetenschappelijke kennis die voor beleidsmakers beschikbaar is. (Onderzoek naar bepaalde ziektes of behandelingen wordt, bijvoorbeeld, eenvoudig niet gefinancierd, en dus niet uitgevoerd omdat de patiëntengroepen te klein zijn of niet interessant genoeg.) Deze externe afhankelijkheid maakt besluitvormingsprocessen van beleidsmakers zoals het Zorginstituut kwetsbaar, omdat ze gemakkelijk mogelijk 'epistemisch onrecht' dat elders is ontstaan, overnemen. 
Tot slot In het laatste hoofdstuk stelt de auteur dat het aankaarten van 'epistemisch onrecht' in besluitvormingspraktijken meer vergt dan een kritische analyse van het kenniswerk van beleidsmakers alleen. Het vergt een analyse op het niveau van de (inter)nationale kennisinfrastructuur waarin beleidsmakers opereren. Volgens de auteur is een analyse van rechtszaken, publieke controverses en sociale conflicten, zoals die gegeven is in dit boek, een goede manier om gevallen van 'epistemisch onrecht' op dit niveau te identificeren. Dergelijke fricties, die wellicht als lastig worden ervaren door de betrokkenen, zijn een uitgelezen kans om te leren over vooroordelen en 'epistemisch onrecht' in kennispraktijken van beleidsmakers die juist beogen objectief en onpartijdig te zijn en onrechtvaardigheid te voorkomen.

De 'idioot' in de titel van dit boek verwijst naar iemand die systemen, conventies en dagelijkse routines ontregelt. Net als in het boek van Dostojevski, 'The Idiot', verwijst de term naar iemand die, met zijn verwarde aanwezigheid, de dominantie van maatschappelijke waarden, normen en conventies toont en ter discussie stelt. Zo'n persoon is niet 'dom' of 'iemand met een lage intelligentie' (zoals de term vaak wordt gedefinieerd in moderne woordenboeken) maar iemand die simpelweg ontregelt. In het oude Griekenland werd de term 'idioot' gebruikt voor iemand die de taal van de polis niet sprak. De 'idioot' is diegene die consensusvorming vertraagt, omdat hij de legitimiteit van het politieke idioom ter discussie stelt. Zoals blijkt uit dit boek, kunnen we van 'idioten' die onze normen voor 'kennen' afwijzen veel leren over het 'epistemisch onrecht' dat mogelijk in onze kennispraktijken circuleert. 


\section{List of Publications}

Moes, F., Houwaart, E., Delnoij, D. \& Horstman, K. (forthcoming). Questions regarding 'Epistemic Injustice' in knowledge-intensive policymaking: two examples from Dutch health insurance policy. [Revisions submitted]

Moes, F., Houwaart, E., Delnoij, D. \& Horstman, K. (forthcoming). Collective Constructions of 'Waste': Epistemic practices for disinvestment in the context of Dutch Social Health Insurance. [Revisions submitted]

Moes, F., Houwaart, E., Delnoij, D. \& Horstman, K. (2018). Strangers in the ER: Quality indicators and third party interference in Dutch emergency care. Journal of Evaluation in Clinical Practice,1-8.

Horstman, K. \& Moes, F. (2018). Wat is dat eigenlijk, context? Spectrum. Tijdschrift voor gezondheidswetenschappen (TSG) 1/ 2018

Moes, F. (March 2017). Televisie voor Sociologen: 'O.J. Made in America' Sociologie Magazine. 25(1), 22-24

Moes, F., Houwaart, E., Delnoij, D. \& Horstman, K. (2016). Contested evidence: a Dutch reimbursement decision taken to court. Health Economics, Policy and Law, 12(3), 325-344

Moes, F. (September 2016). Blauwdruk van een omgekeerde wereld. Sociologie Magazine. 24(3), $10-11$

Moes, F. (2016). De stad in actie. Actor Netwerk Theorie. Van Es, R. \& Van Rossum, J. [red]. De stad als organisatie. Lessen uit The Wire. Deventer: Vakmedianet. 82-92

Moes, F. (2016). De 'Stat Game'. De verborgen krachten van de statistiek. Van Es, R \& Van Rossum, J. [red]. De stad als organisatie. Lessen uit The Wire. Deventer: Vakmedianet. 134-150

Moes, F. (2015). 'All the pieces matter'- Latours slowciologie en The Wire. In Van Oorschot, I., M'Charek, A., Harambam, J. \& Benschop, R. [red]. Monsters in de Sociologie. De wondere wereld van ANT. Boom/ Lemma, Amsterdam. 28-45

Moes, F. (2014). 'All the pieces matter'- Latours slowciologie en The Wire. Sociologie. 10(3). 242259 



\section{Acknowledgements}

From the outside, writing a dissertation may seem like a long and lonely process. There are many reasons why my experience of the past few years does not fit that description. These 'reasons' are the people that accompanied me along the way. I would like to extend a heartfelt word of thanks to a number of people in particular, but these few lines of acknowledgement can barely do them justice and this word of thanks is - inevitably - incomplete.

First of all, I am much indebted to my team of supervisors. I am very grateful for the animated supervision of Prof. Klasien Horstman, who was not only an inspiring guidance regarding the empirical content and conceptual framing of this book. She was also, and continues to be, a tremendous moral support. Furthermore, I was blessed to have Prof. Diana Delnoij in my supervisory team, who helped me focus my empirical research. She has been a great support, especially in helping me find my way at the National Health Care Institute where she heads the Quality Institute. The team would not have been complete without its historical conscience, Prof. Eddy Houwaart, who provided the much needed socio-historical context to my work.

Over the past few years, staff, board and expert committee members working at the National Health Care Institute (Zorginstituut Nederland) allowed me to observe their daily work. This study would not have been possible without their hospitability and tolerance. I gratefully acknowledge the contributions made by all research participants. I am especially thankful to those who gave up their precious time for interviews and/or critical reflection on my research and to those who shared their workspace and lunch time with me, treating me as one of their own. I would also like to express my sincere gratitude to the former board of directors of the Institute - Arnold Moerkamp, Bert Boer, and Marian Grobbink - and to the managers Maud van der Veen, Arnold van Halteren, and Diana Delnoij for their continuous support of my study. The members of the Institute's research group 'Rechtvaardiging en Draagvlak Zorginstituut' (REDZ) - Payam Abrishami, Reinoud Doeschot, Pé Mullenders, Jacqueline Zwaap, Edmar Weitenberg, and Sander van de Scheur - I would like to offer you my thanks for shaping the contours of the research project and facilitating it. I additionally want to express my gratitude to Christene Beddow, who was always ready to edit my work, even under severe time pressure.

My colleagues at the department of Health, Ethics \& Society, Maastricht University - in particular Mare Knibbe, Dorothee Hortskötter, Lucie Bastiaens, Wang Li, Rob Houtepen, Greg Stapleton, Bart Penders, Agnes Meershoek, Nora Engel, Els Geelen, Olga Zvonareva, Lloyd Akrong, Birgit Wouters, Anna Wolters, Gili Yaron, Marjolein de Boer, Inge Lecluijze, Cristina Quevedo, Sjoerd Cratsborn, Wybo Dondorp, Guido de Wert, Jenny Slatman, Hellen Heutz, Angelique Heijnen, and Kyra Kramer - gave me continuous support, academic reflection, discussion, and insights. I am very grateful for having such a strong and warm academic backing, especially because I am not at the office on a daily basis. Thank you, Nora Engel, Cristina Quevedo, Willemine Willems, Felipe 
Wiens, Joost de Vries, Mare Knibbe and Emke Bosgraaf for sharing your houses, dinners, and breakfasts with me when I was in Maastricht.

It is the practice of yoga that takes me out of my academic head and puts me on my earthly feet every day. This practice keeps me stable and I am convinced that it has benefited my academic work in some way or another. I am, therefore, very grateful for the inspiring teachings of Berber Schönholzer. I am lucky to teach with some of the finest yoga teachers at her studio in Amsterdam lyengar Yoga Zeeburg. At Yoga Maastricht, the lovely old hat-factory-turned-yoga-studio of Melanie Tillema and her team, I was given the opportunity to teach my first classes. I have learned a lot from both Melanie and Berber, not only about the nourishing qualities of lyengar yoga and how to conduct a yoga class, but also how to run a warm, inspiring, and welcoming yoga studio. I am much indebted, furthermore, to Pien Wijn, for her invaluable wisdom, moral support, and personal guidance.

In Amsterdam I share an office with a group of creative, committed change-makers in sustainability. Thank you - Mette te Velde and Roos de Jager (Strawberry Earth), Raoul Kuiper and Scato van Opstall (Karma Brothers), Brechtje Baars and Leonoor Verplanken (The Greenhouse), Nard Haverkort, Christiaan Maats, Erik Grootscholte, Lizet Deutekom, Jochem Duyff, Kim van Dongen, Marianne van der Heijden, and Annebeth Erdbrink- for the family lunches, moral support and the daily dose of comedy. I am very thankful for the cover of this book, designed by Christiaan Maats.

I am eternally grateful for the unconditional support of my parents, Hank and Sabine Moes, my brothers, Daan and Rein Moes (who agreed to be my paranymphs), and my friends - in particular Barbara Smit, Margriet van Lidth de Jeude, Isabelle van 't Groenewout, Wendele Osterthun, Pieternel Osinga and Sabrina Brandt. Marijn Korff de Gidts, there are no words to express the gratitude I feel for the life that we share (and if these words existed, I doubt whether this page would be the place to express them), but: here's to the love we share, and here's to our unborn child! The greatest lessons in life we have yet to learn, and I am deeply grateful to have you by my side. 




\section{Author Details}

Floortje Moes (1983) is affiliated, as post-doctoral researcher, with the department of Health, Ethics \& Society (Faculty of Health, Medicine and Life Sciences) of Maastricht University. Before starting her PhD research into the knowledge work of the Dutch National Health Care Institute (Zorginstituut Nederland) in 2013, she worked as a research fellow for the Scientific Council for Government Policy in The Hague (Wetenschappelijke Raad voor het Regeringsbeleid, WRR) and as a teacher of Philosophy of Science at the University of Amsterdam. Since 2015, Floortje also works part-time as a certified lyengar Yoga teacher.

Floortje obtained a Master's degree in Philosophy from the

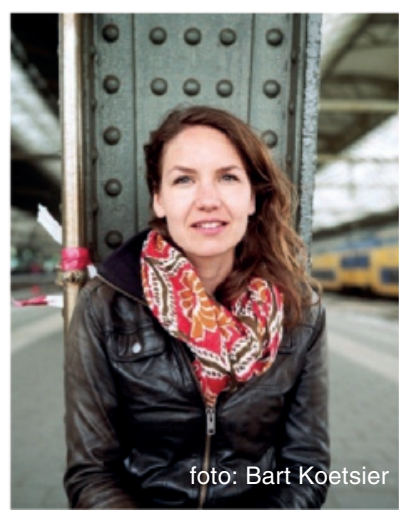
University of Amsterdam (cum laude, 2009), a Master's degree in Conflict Studies \& Human Rights from Utrecht University (cum laude, 2009), a Bachelor's degree in Philosophy from the University of Amsterdam (2008) and a Bachelor's degree in International Business \& Languages from the University of Applied Sciences in Amsterdam (2007). As part of her studies, Floortje spent several semesters attending courses at Stellenbosch University (Stellenbosch, South-Africa, 2001), Universidad de Santiago de Chile (Santiago, Chile, 2005) and Ibmec (Rio de Janeiro, Brazil, 2006).

As post-doctoral researcher, Floortje currently continues her academic work in the epistemology of health care at Maastricht University. Apart from her work at the intersection of medicine, health policy and the philosophy of science, Floortje has developed a range of academic courses, public lectures and (lay) publications in which she uses popular film and documentaries (e.g. HBO's tv-series 'The Wire' and the documentary 'O.J.: Made in America') to bring philosophical reflection to audiences primarily engaged in arts, culture and (urban) sociology. 


In liberal democracies, governments are expected to justify their actions on the basis of scientific and technical knowledge. But whose knowledge and what types of evidence are actually counted as relevant, credible and trustworthy contributions to policymaking processes? This book explores how public accountability for thorny policy choices is constituted in policymakers' epistemic practices: their ways of using, assessing and warranting different types of knowledge to substantiate complex policy decisions. It does so by means of an ethnographic study at the Dutch National Health Care Institute (Zorginstituut Nederland) - an authoritative governing agency in Dutch social health insurance that is tasked to ensure the affordability and accessibility of good quality health care for all citizens.

Engaging with the question of 'epistemic injustice' in policymaking - can we do someone (or a group of people) an injustice by not recognizing their testimonies as credible and intelligible contributions to policymaking processes? - this book reflects on issues regarding the adequacy, appropriateness and social inclusiveness of policymakers' practices of knowing. The author argues that lawsuits, public controversies and social conflicts about policy decisions, that are generally considered sensitive issues for public institutions such as the National Health Care Institute, are actually windows of opportunity to learn about biases, prejudices and other epistemic improprieties that potentially circulate in policymakers' practices of knowing. 